\title{
THE COMBI-TARGETING CONCEPT: SYNTHESIS OF STABLE NITROSOUREAS DESIGNED TO INHIBIT THE EPIDERMAL GROWTH FACTOR RECEPTOR (EGFR)
}

Juozas Domarkas, Fabienne Dudouit, Christopher Williams, Qiu Qiyu, Ranjita Banerjee, Fouad Brahimi, Bertrand Jacques Jean-Claude*

Cancer Drug Research Laboratory, Department of Medicine, Division of Medical Oncology, McGill University Health Center/Royal Victoria Hospital, Montreal, H3A1A1, Quebec, Canada

\section{SUPPORTING INFORMATION:}

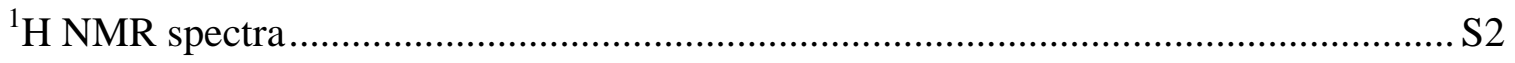

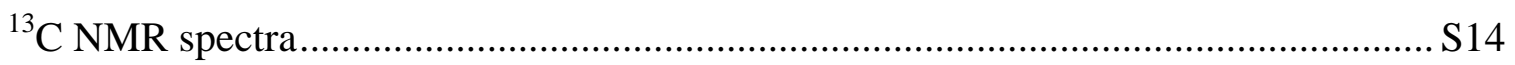

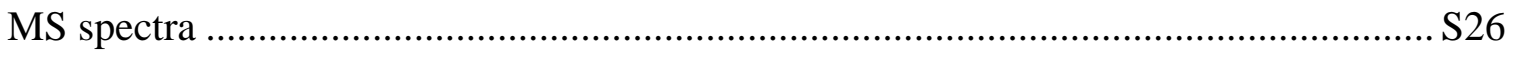

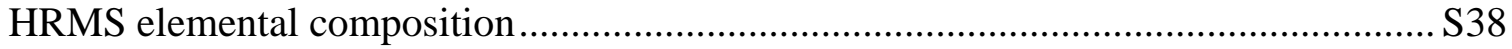

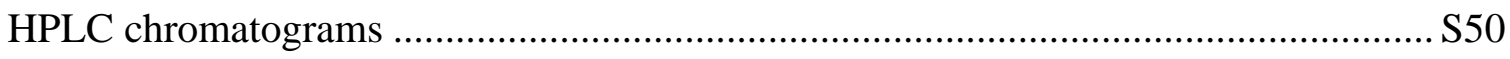




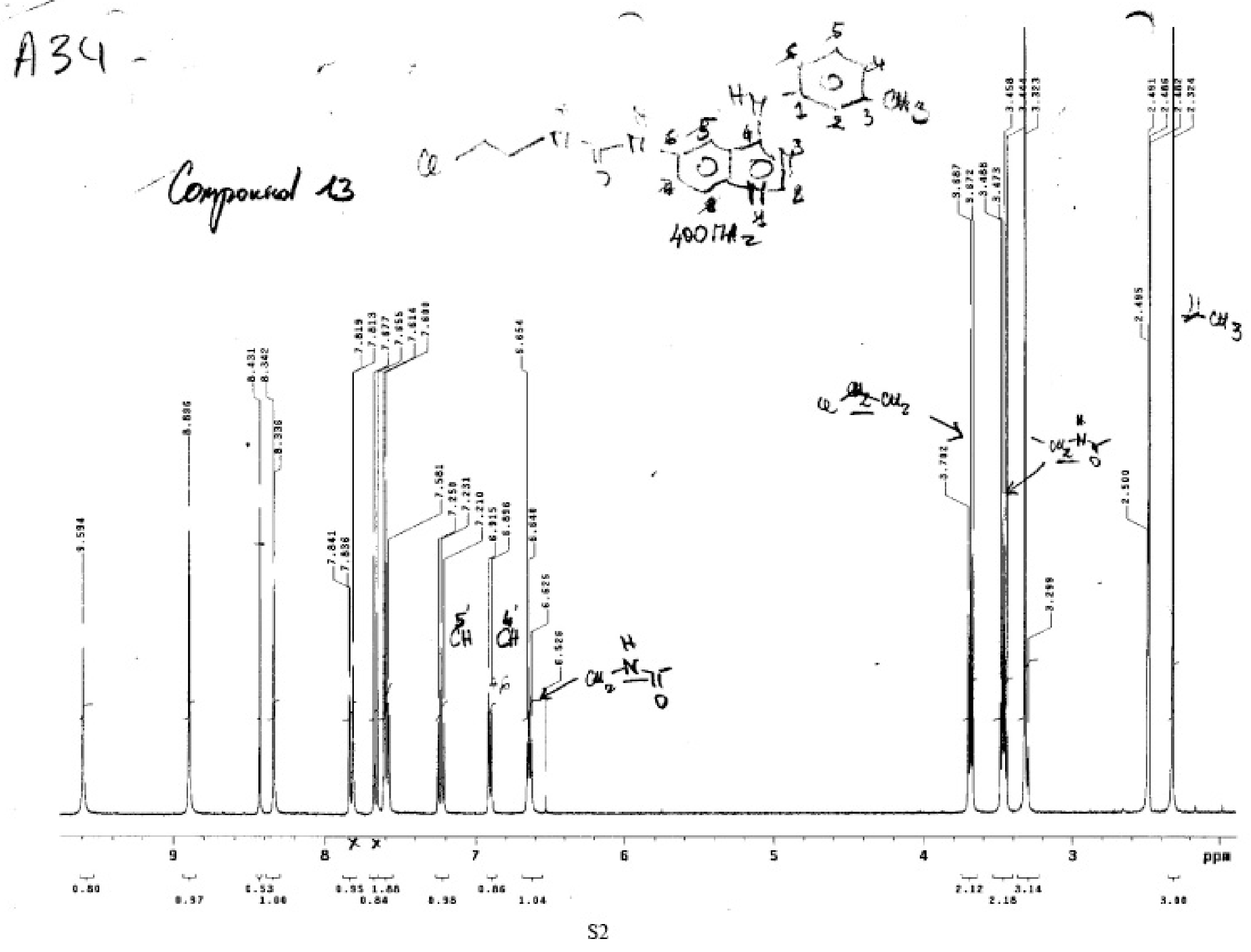




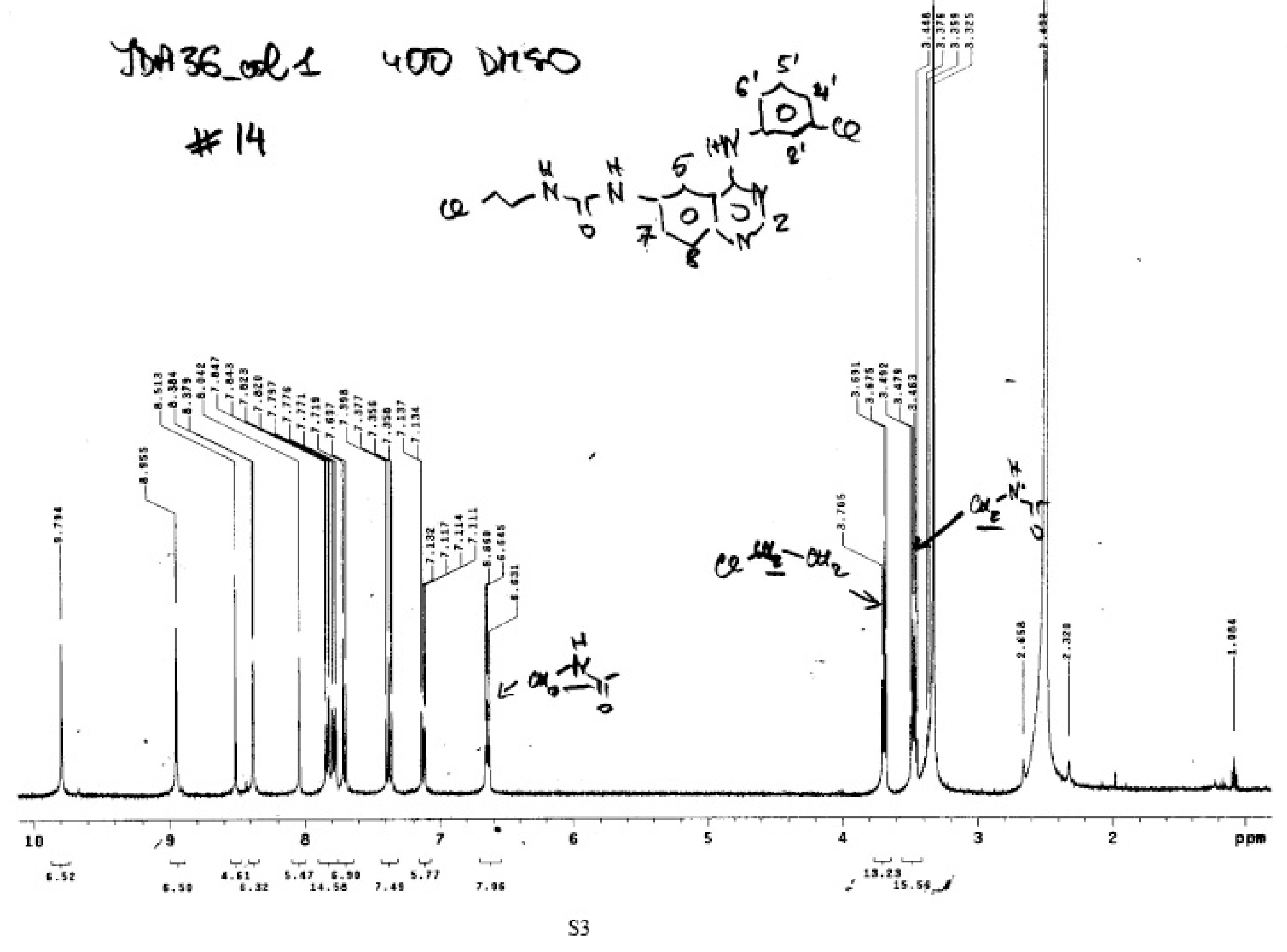


farcorec

A40 15

$+{ }_{H T} \widehat{0}_{B r}$

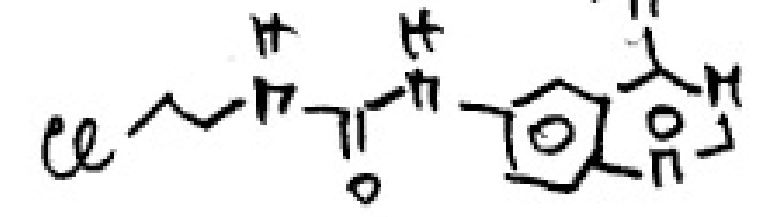

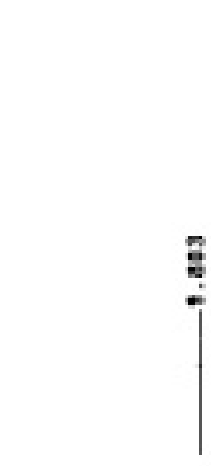

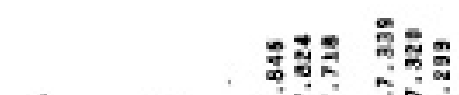

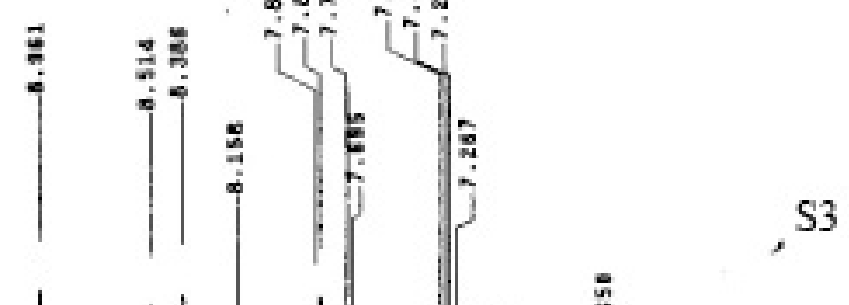

(t.)ll

400
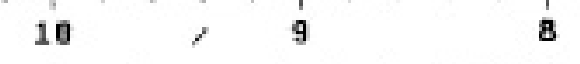

$\underbrace{0.90}_{1.08} 1.97 .98$ 1.86

$\stackrel{0}{u}$

S3

$\stackrel{5.05}{1.5}$

1.

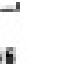

.97 
JDAl42 $400 \mathrm{~Hz}$ JDA 49
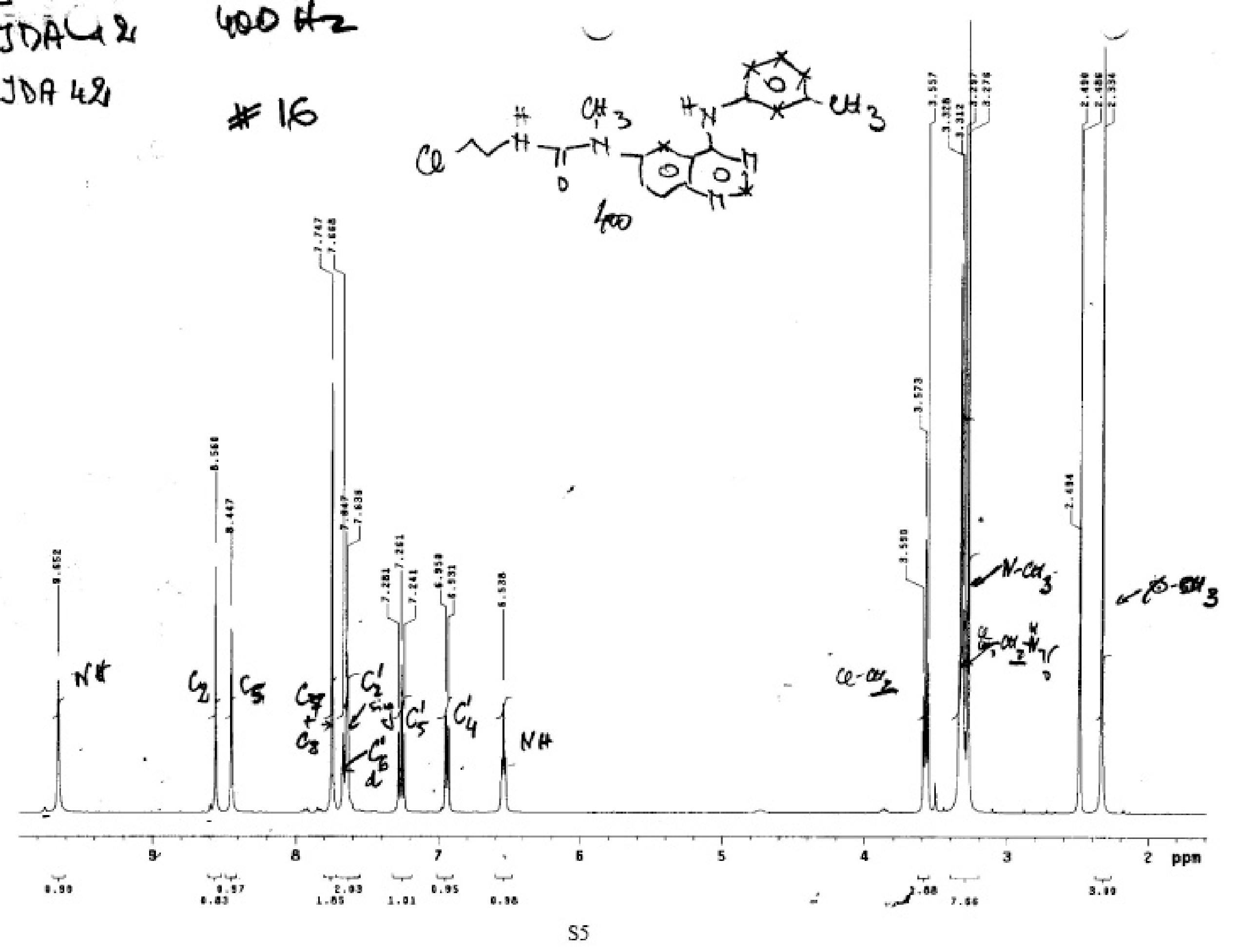
TDALI 400 DISSO

$$
\# 17
$$

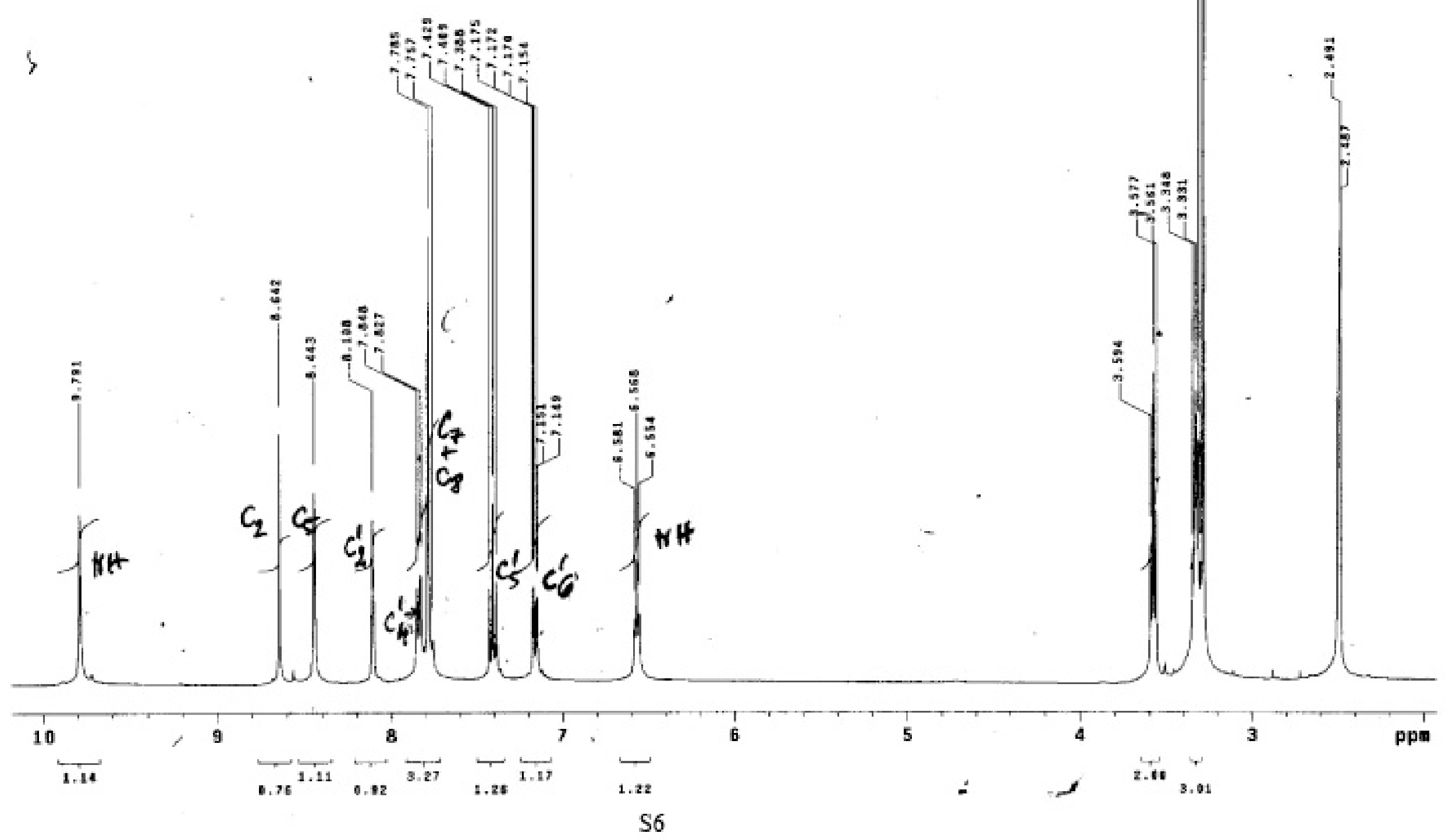



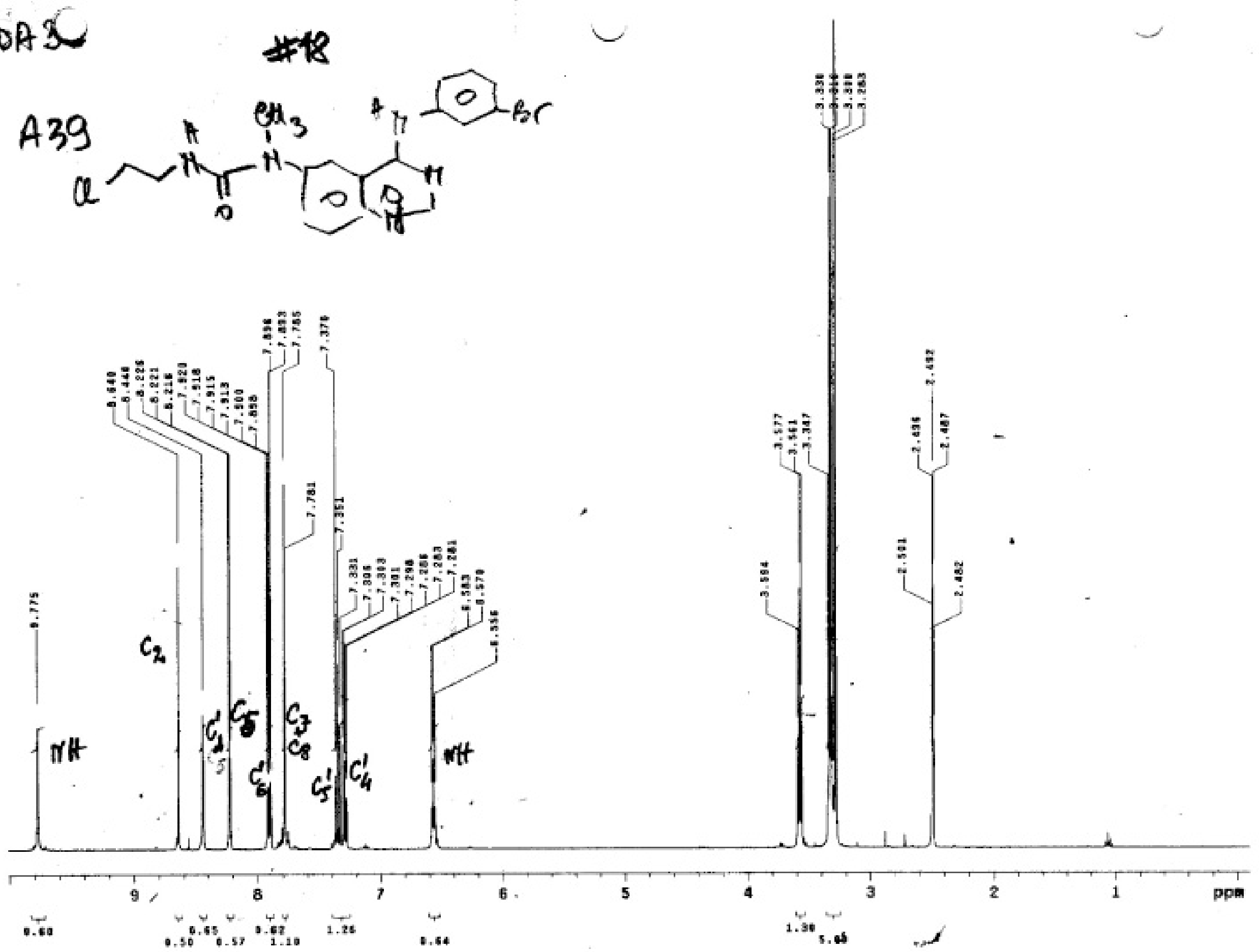
$\longrightarrow$

JDA73 $400 \mathrm{Vtt} / \mathrm{O} 780$

* 19
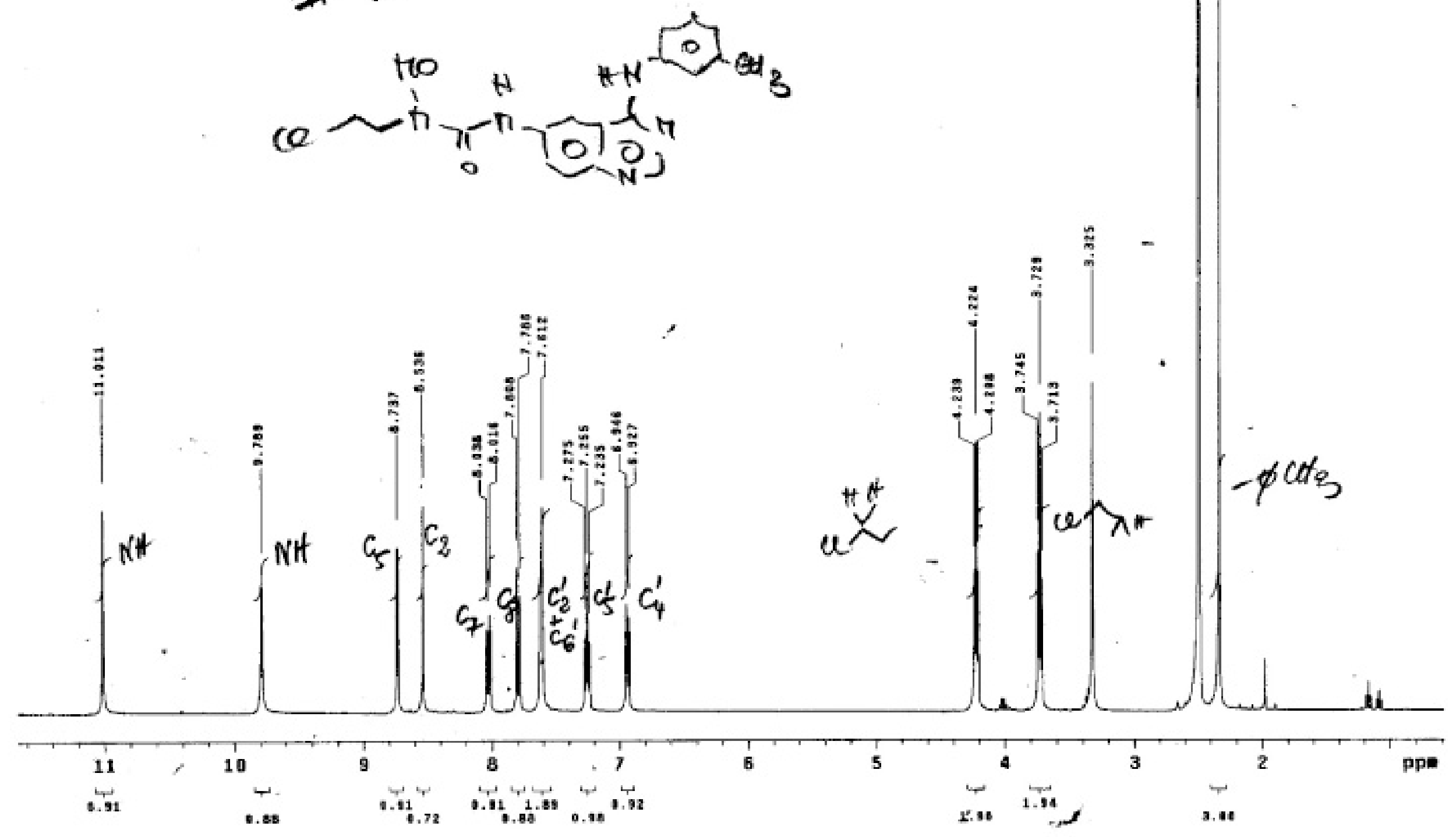

S8 
30965 ख180 $300 \mathrm{Nu}_{2}$
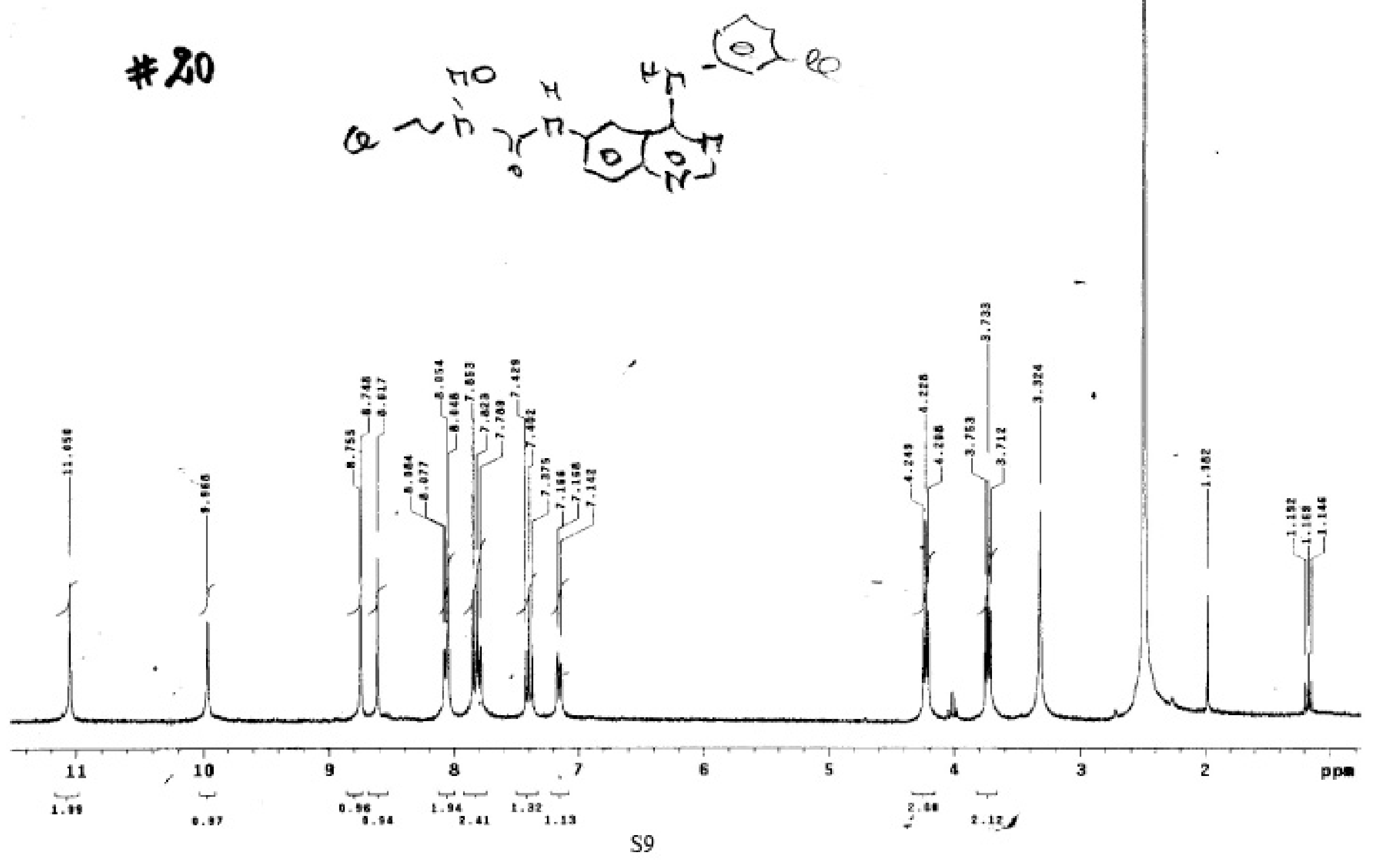
TOAC
$A G 6$

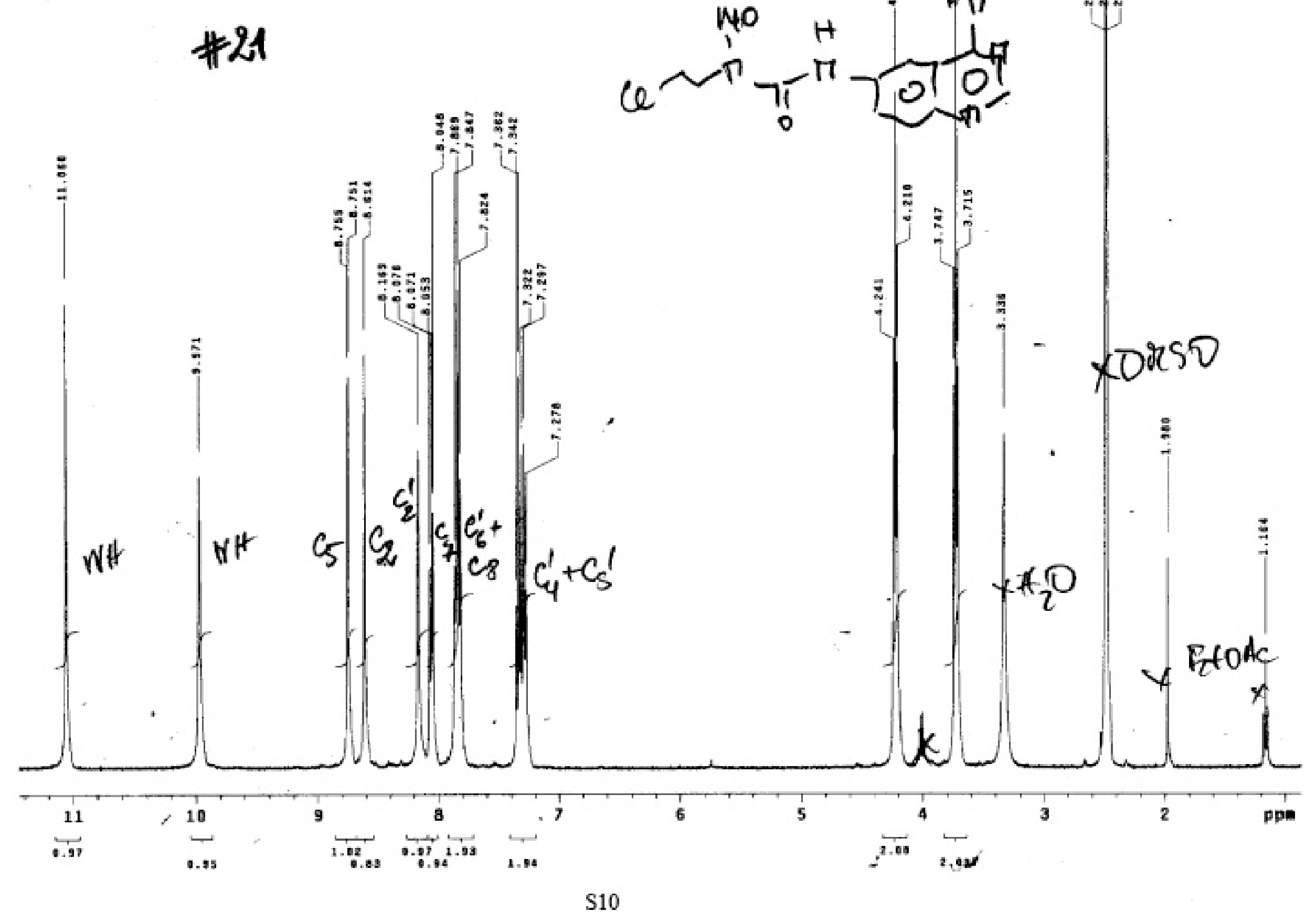


A56

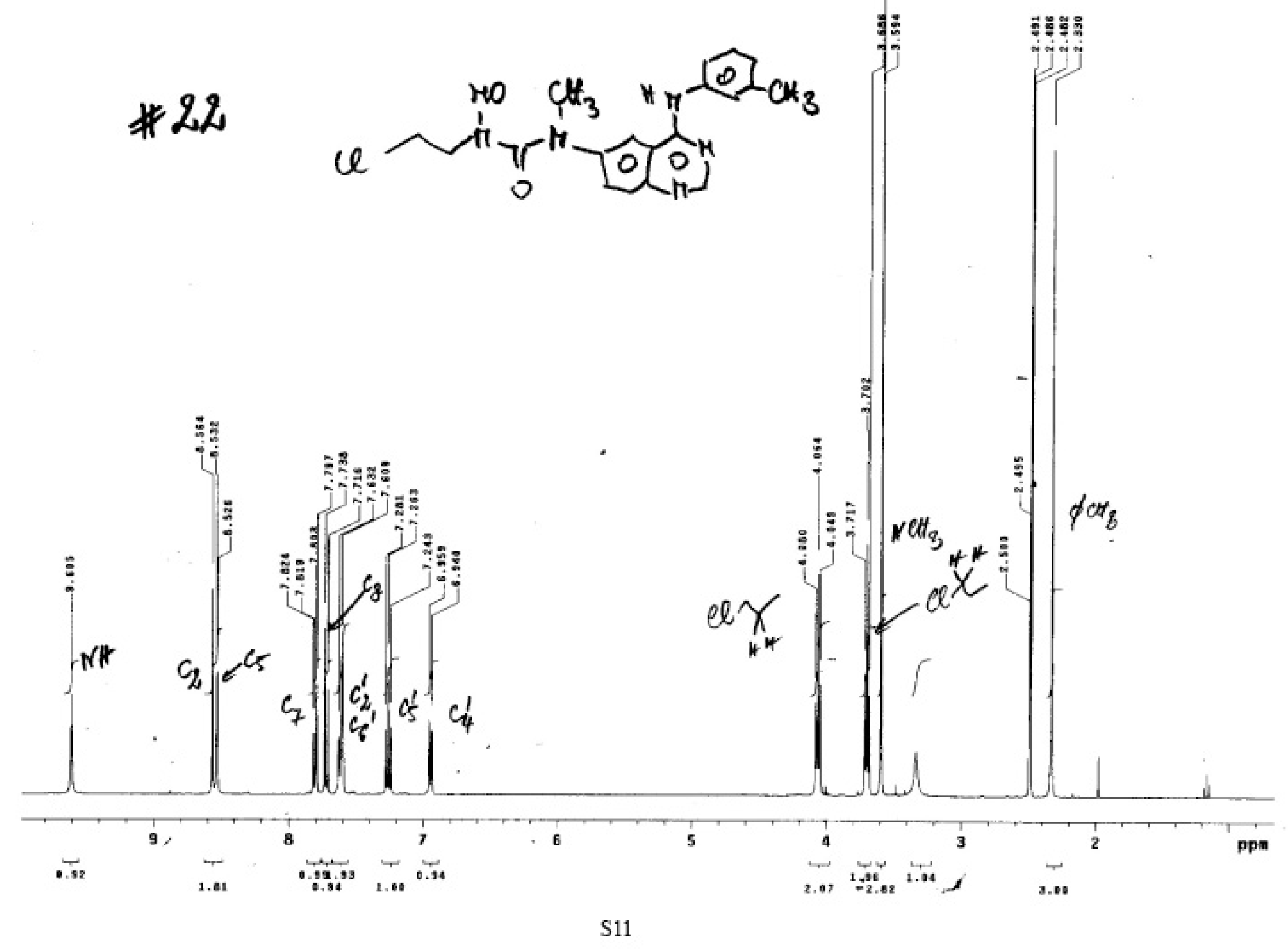




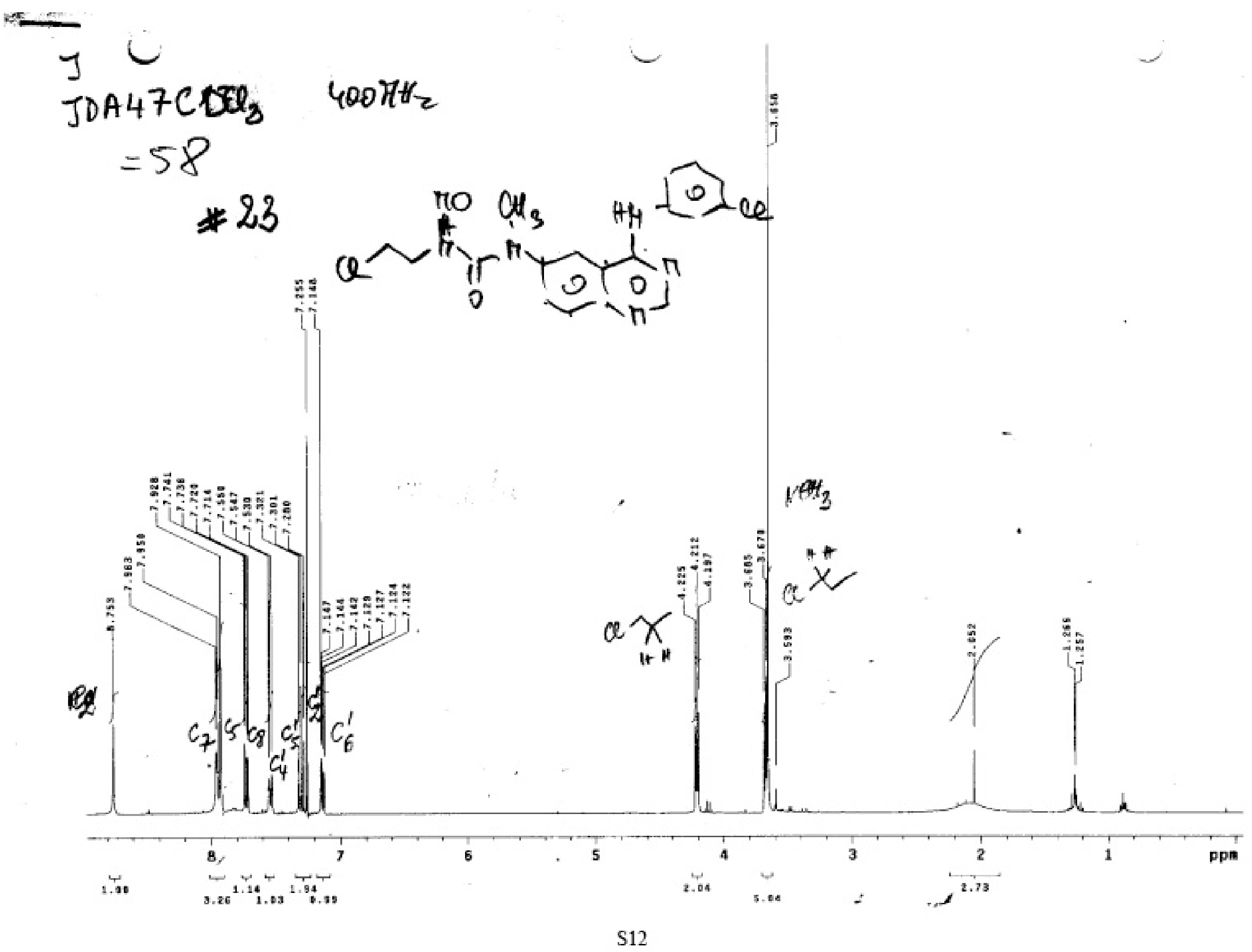




\section{ASQ-t DISO 400}

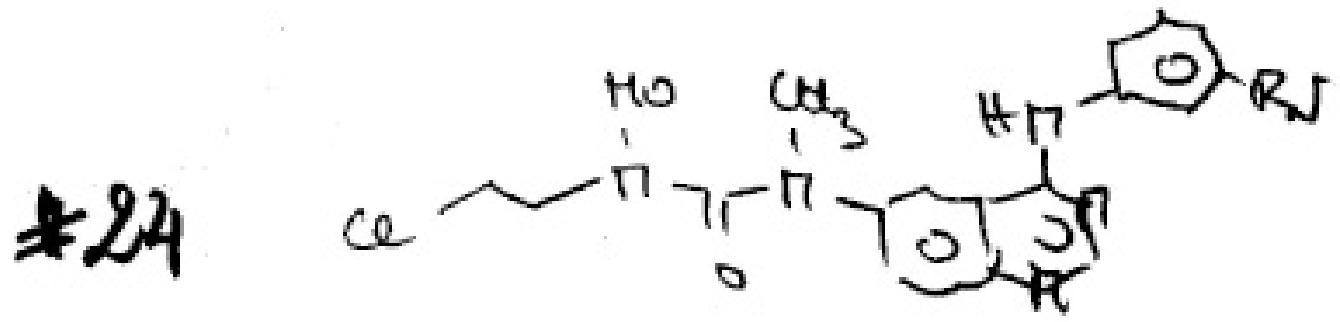
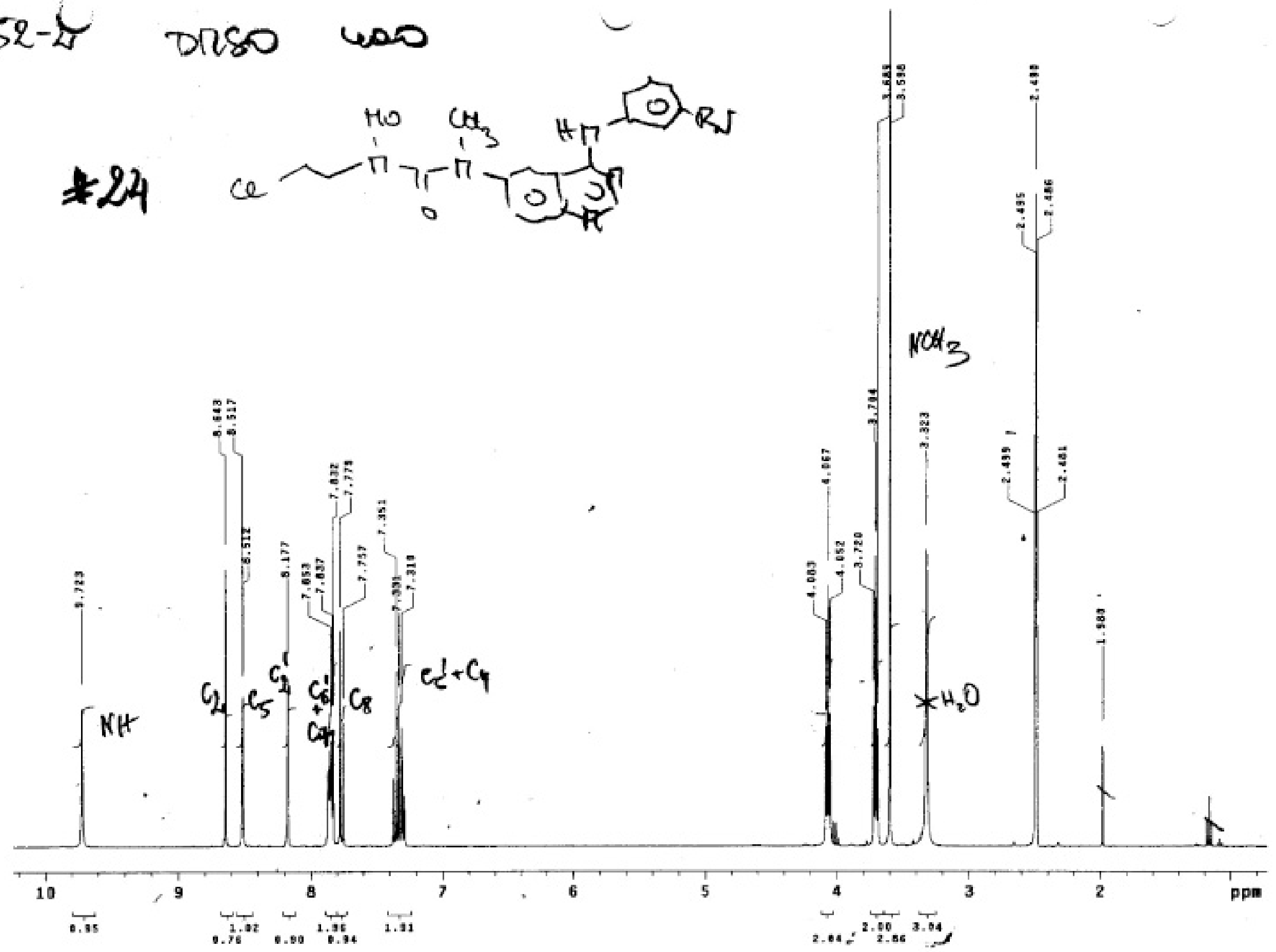


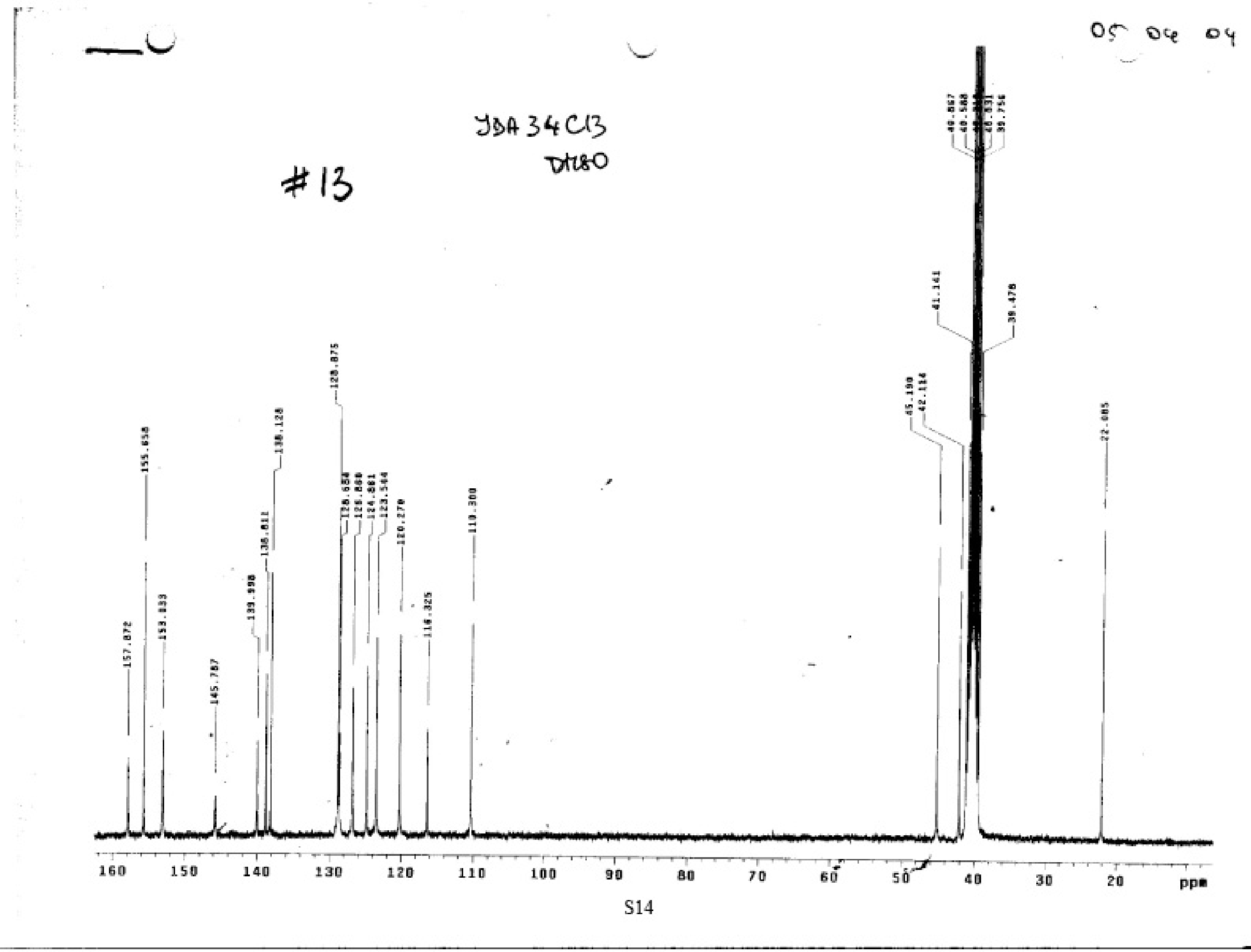


DABS.COIACB DISO 300

\section{\#14}
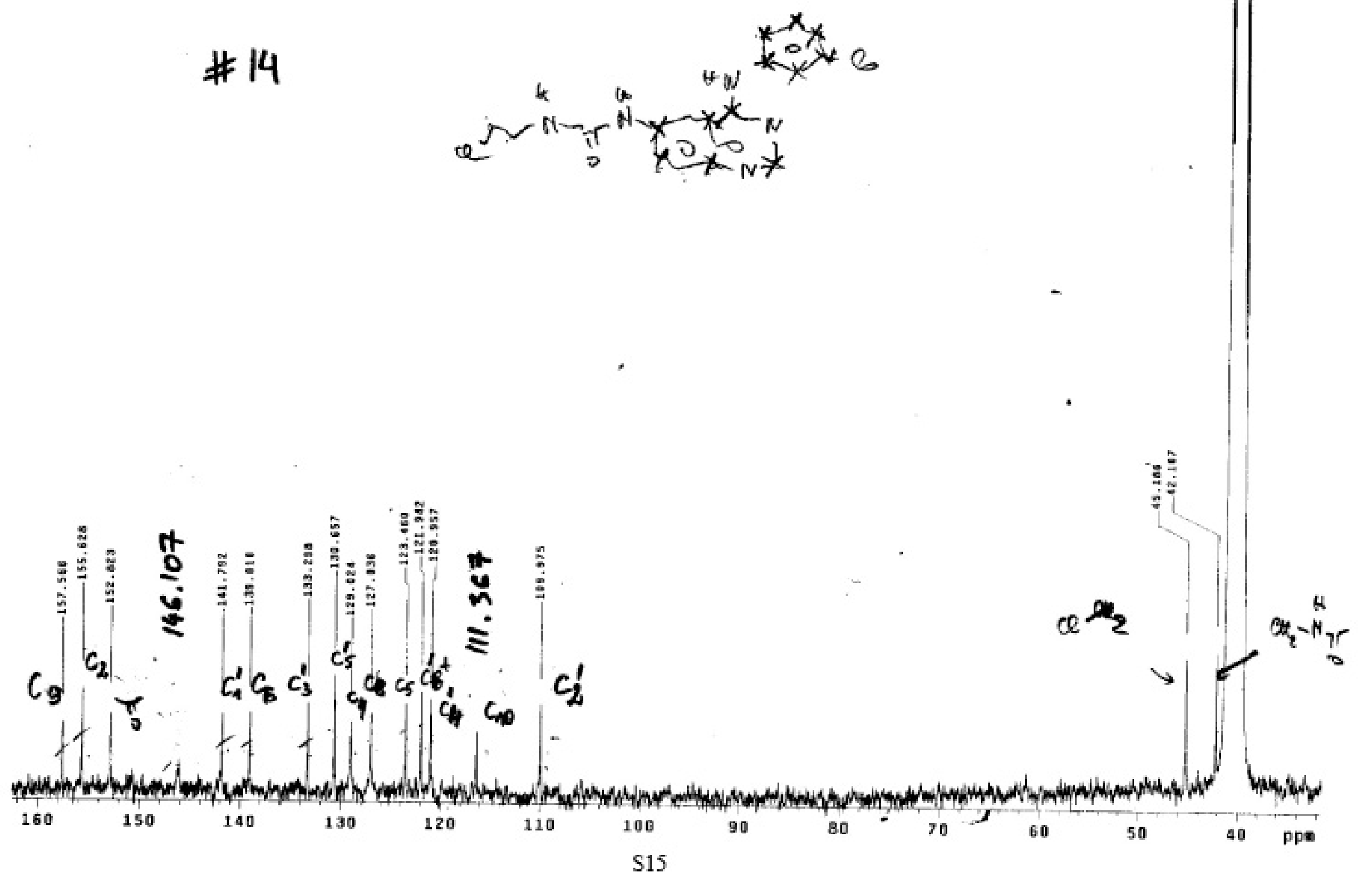



\section{A42C13}

JoA42C13

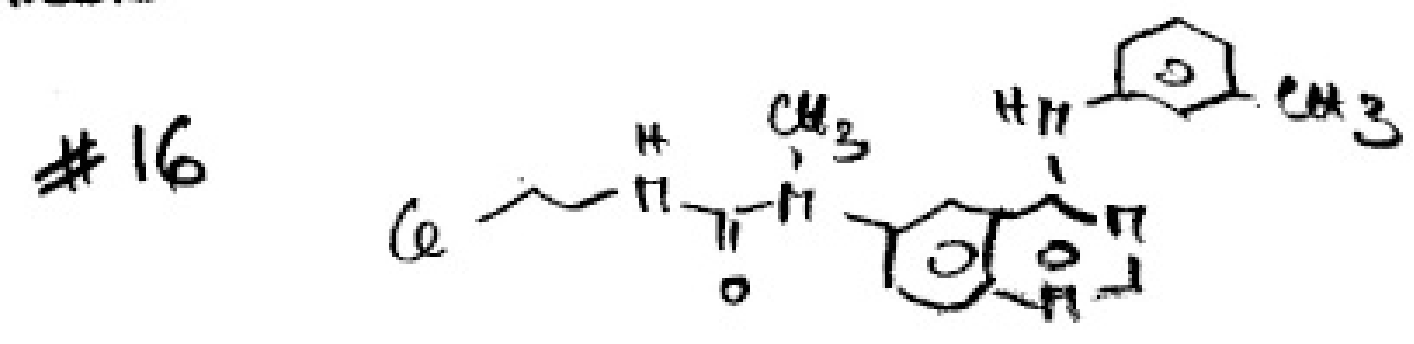

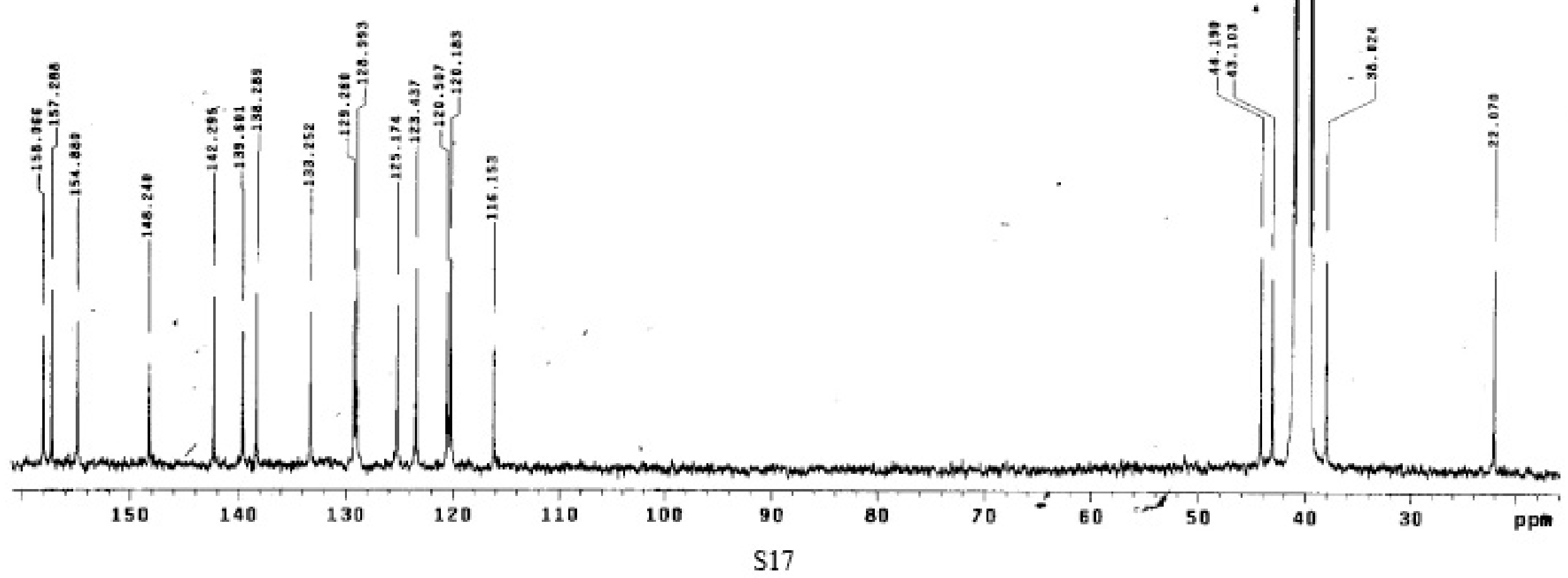




\section{7 \\ JDA4 $=13$}

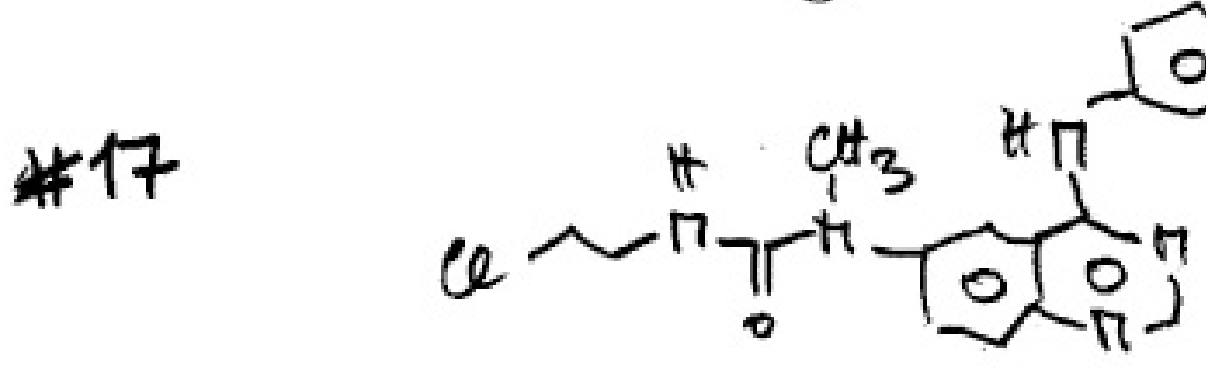

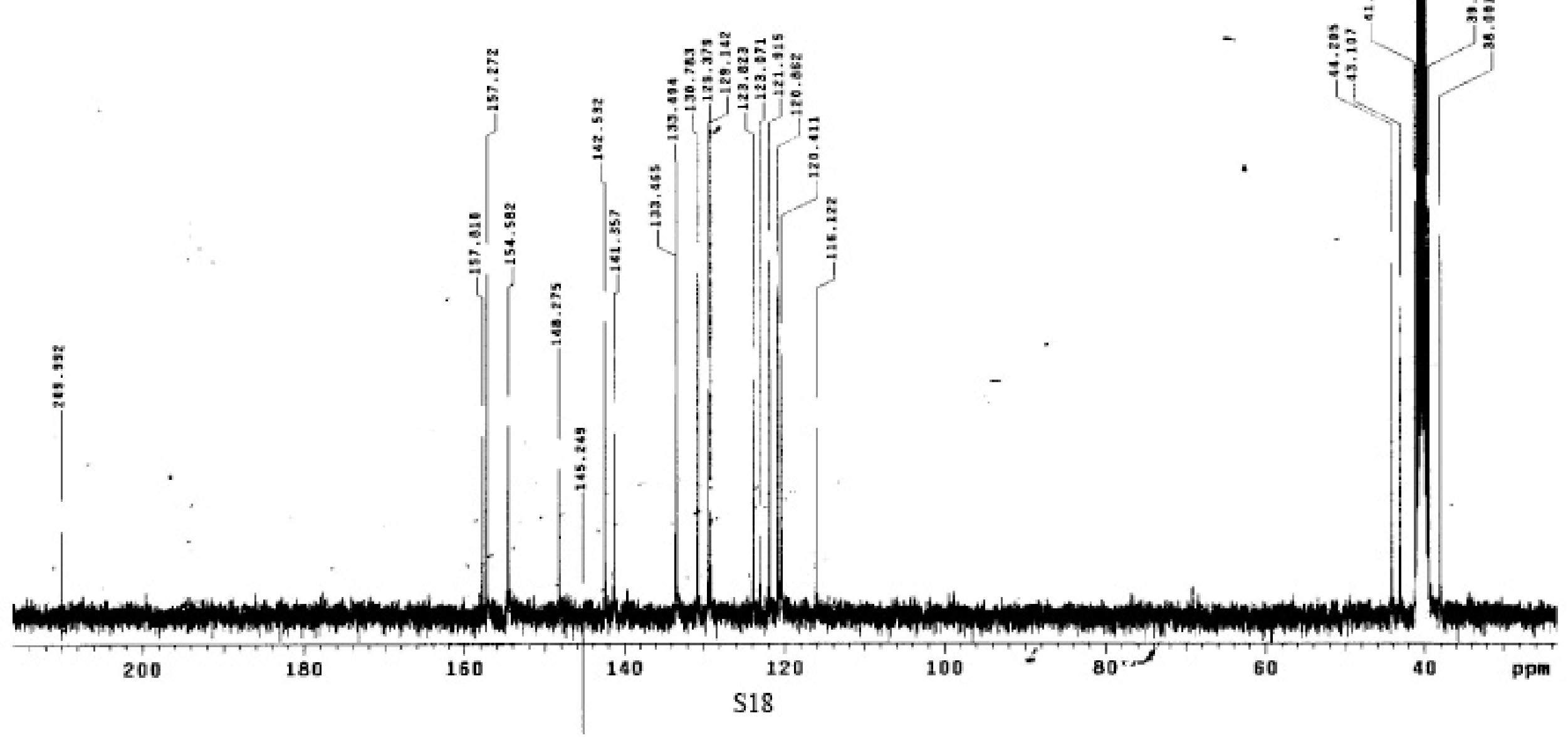




\section{IDAC-2C13}

\section{$139-2013 \# 18$}

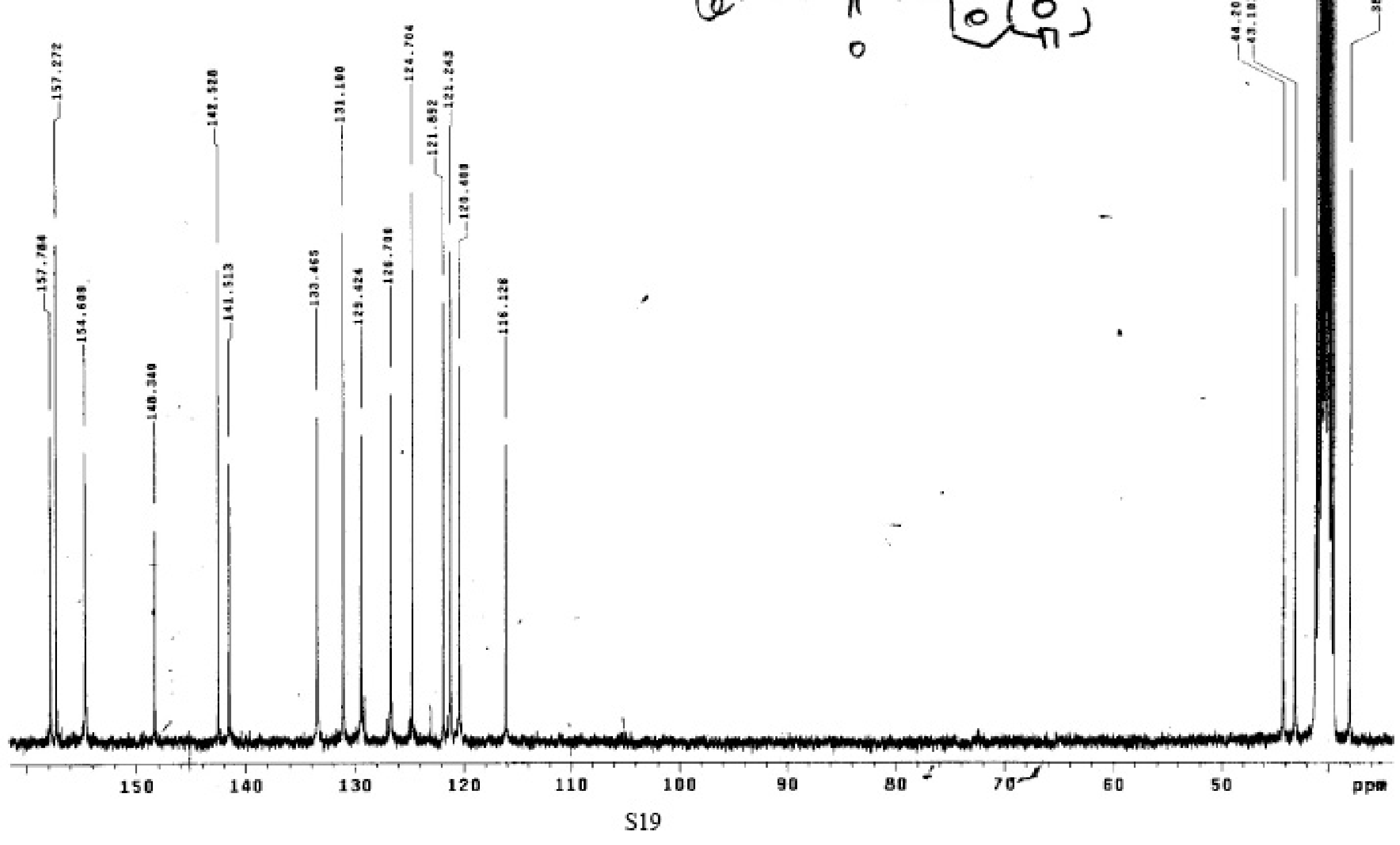


TDA $73 \mathrm{CB}$ OTSO YOMTK \#19

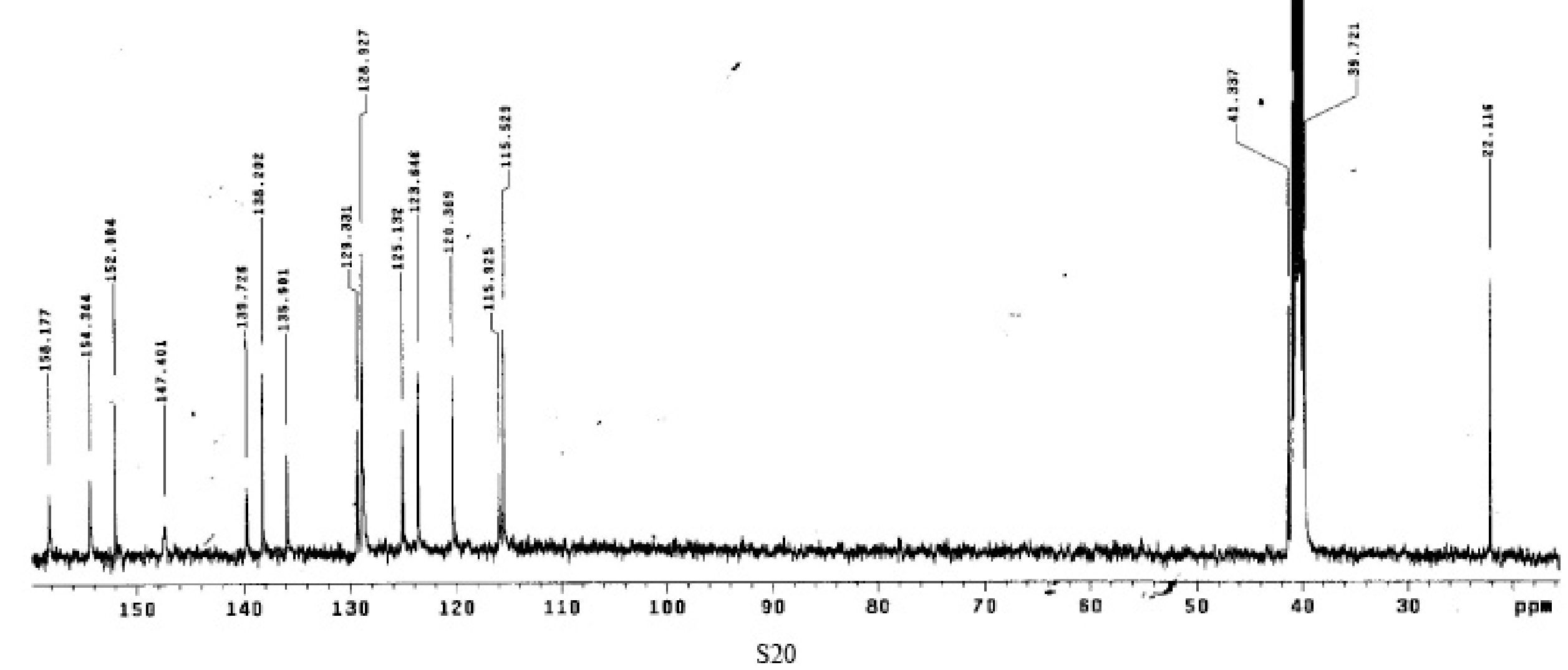




\section{JDA65 chene Cl3 arso soorth $\# 20$}

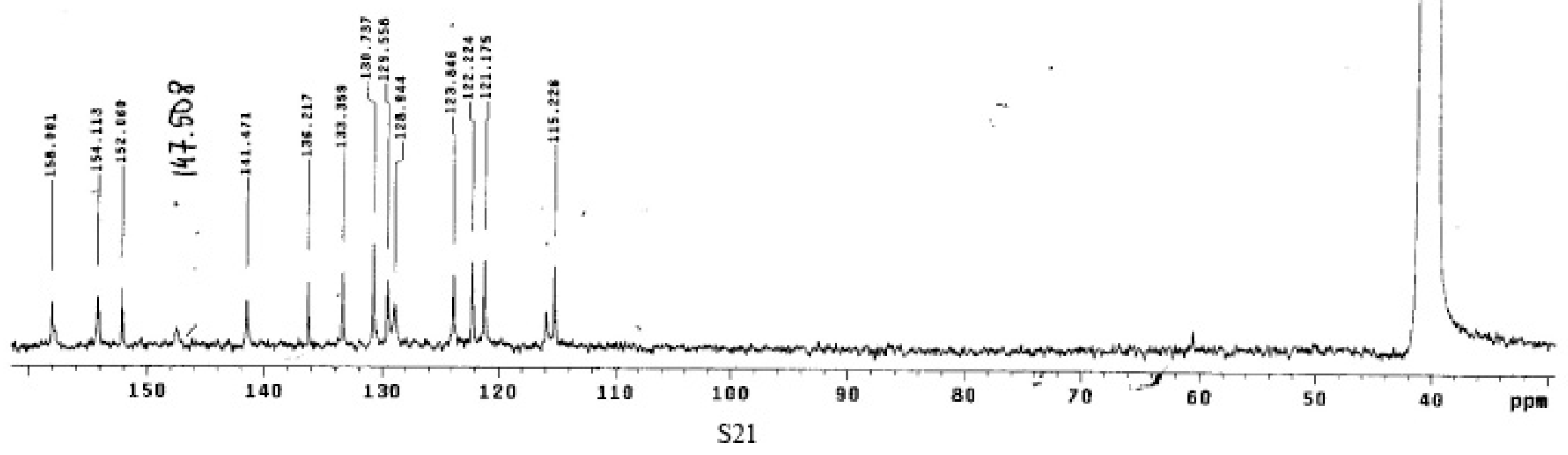


JDA66

Pulse sequence: s2pul

Solvent: 0Mso

Ambient temperatiur

Relax. delay $3.000 \mathrm{sec}$

Pulse 45.9 degrees

Acq. time 1.81 s sec

width $18761.7 \mathrm{~Hz}$
2608 repetitions

(195276 MHz

OECOUPI $\mathrm{H} 1,300.0578578 \mathrm{MHZ}$

cont. inuous ly on

WALT Z - 16 mod du lated

DATA PRQCFSSTNC

I ine Lroadening $1.0 \mathrm{~Hz}$

Total time 112003/ hr, $16 \mathrm{~min}, 7 \mathrm{sec}$
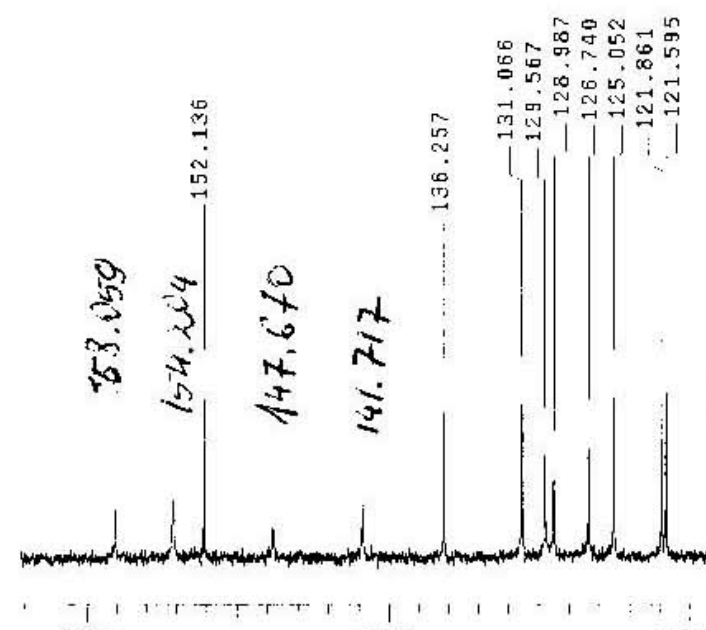

120

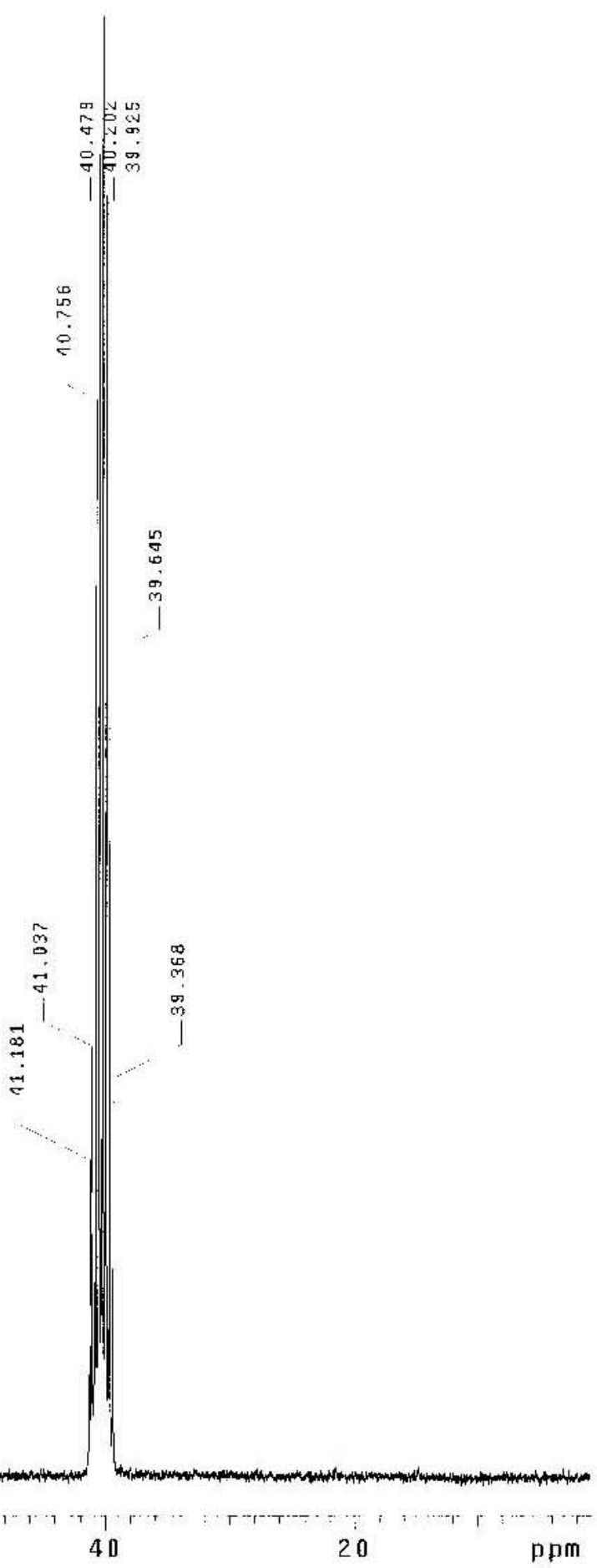


$A 5 G C 13$

\#22
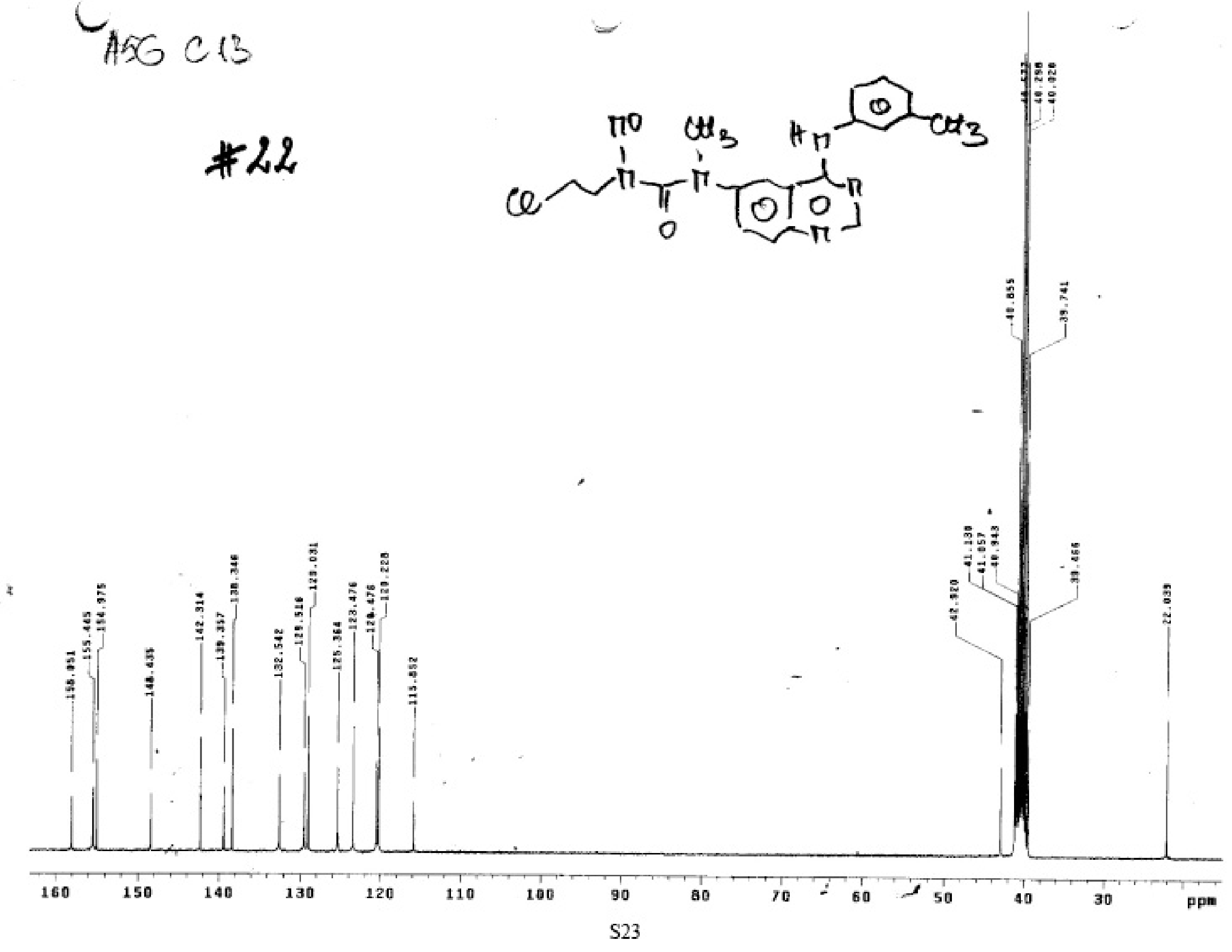


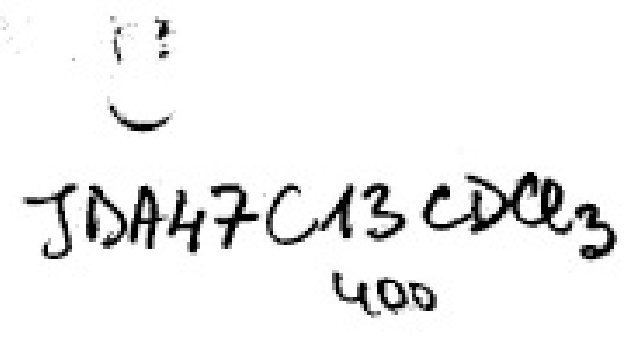

\#23
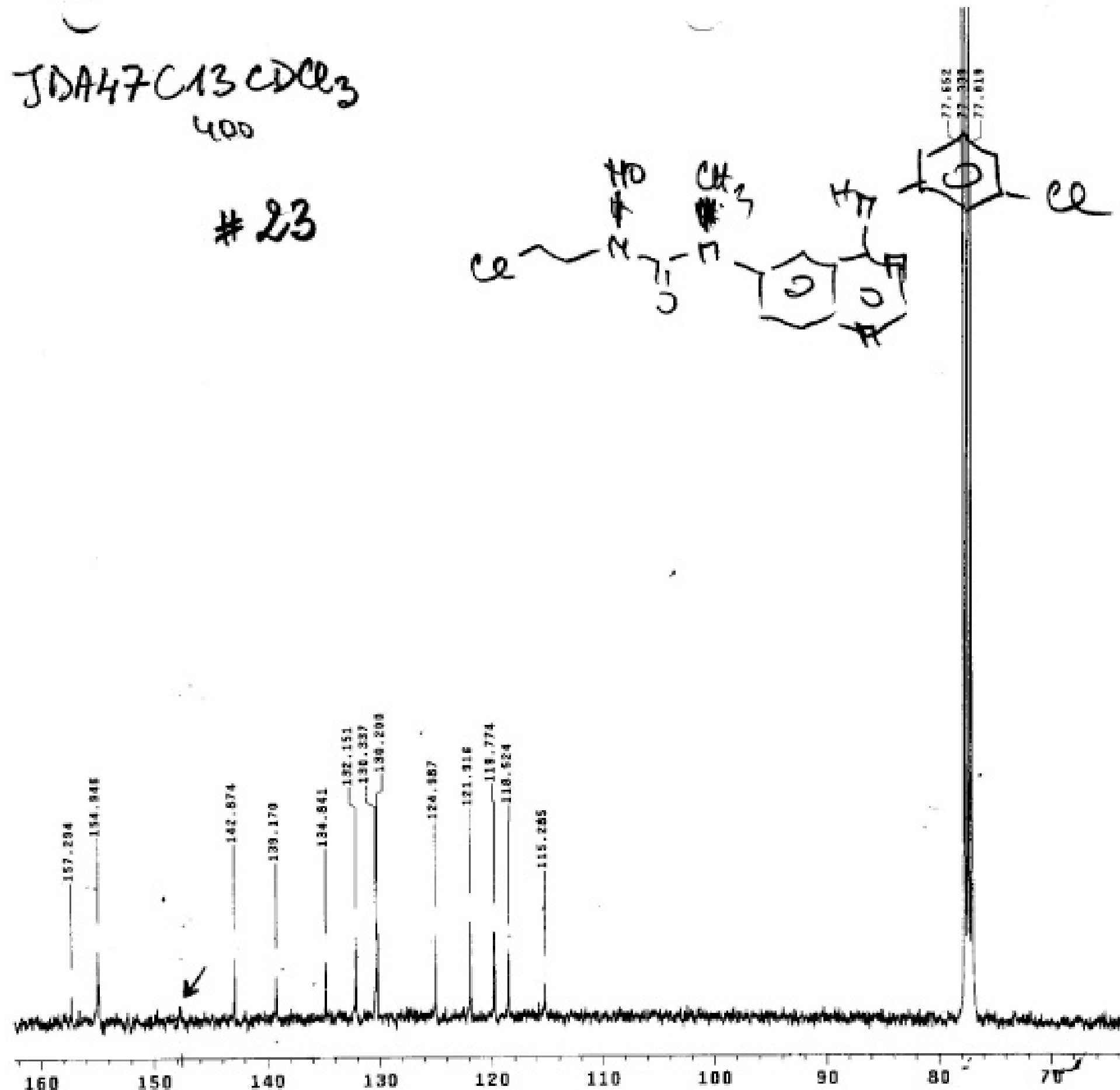

120

110

100

90

80

70 
$\mathrm{AL}_{5} \mathrm{CO}$

A52Cis arso
$\# 24$

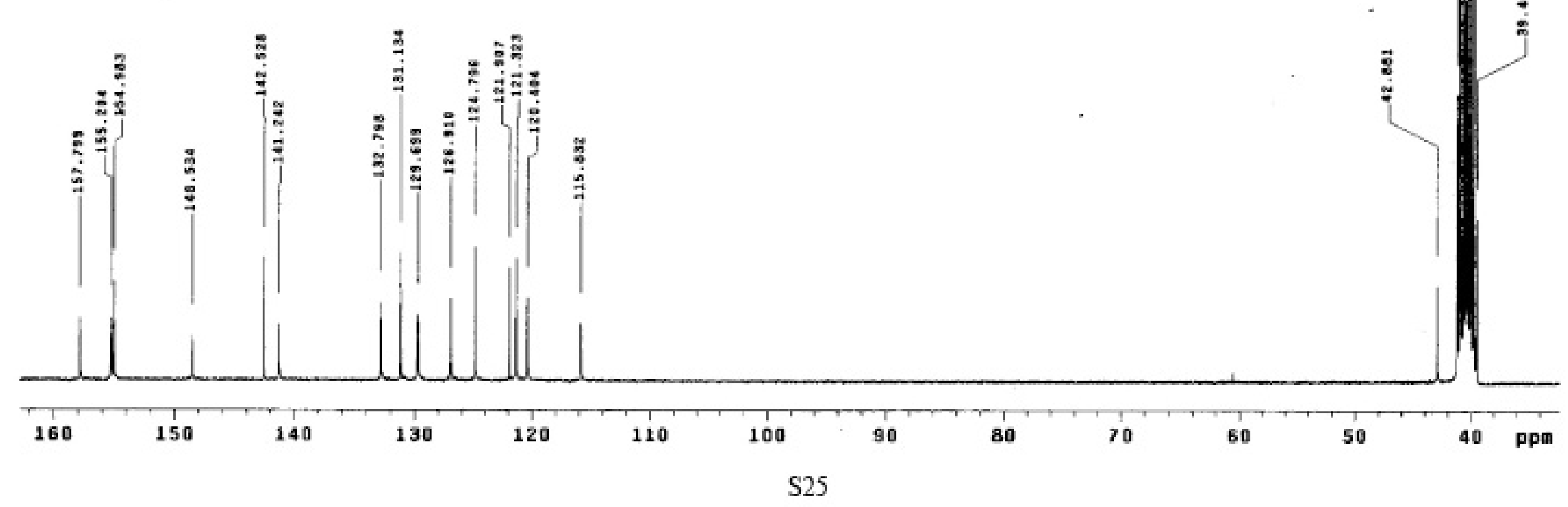




\section{APCI INF.t}

020425_06Aif_DOMARKAS_JDA34 *22-28 RT: 0.71-0.84 AV: 5 NL: 8.35E6

$\mathrm{T}:+c \mathrm{~ms}[$ [50.00-2000.00]

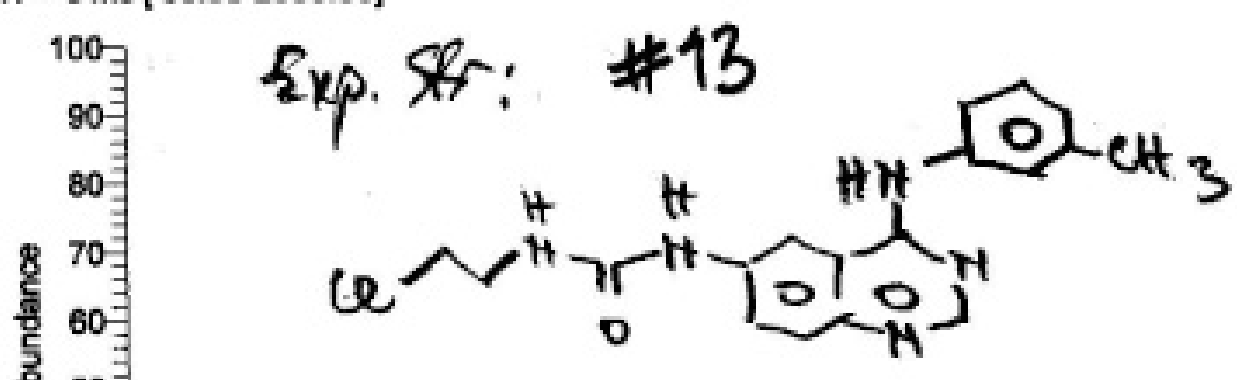

起 50

黄 40

¿ 30

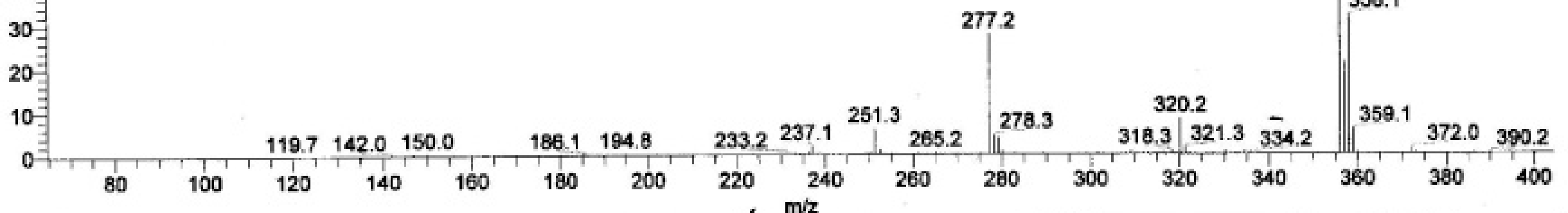

80

$m / z$

\section{5_06Aif_DOMARKAS_JDA34 $142-49$ RT: 1.07-1.15 AV: 8 NL: $5.56 E 7$}

$\mathrm{T} ;+\mathrm{c}$ Full $\mathrm{ms} 2 \overline{2} 356.00 @ 28 . \overline{0} 0$ [ $95.00-376.00]$
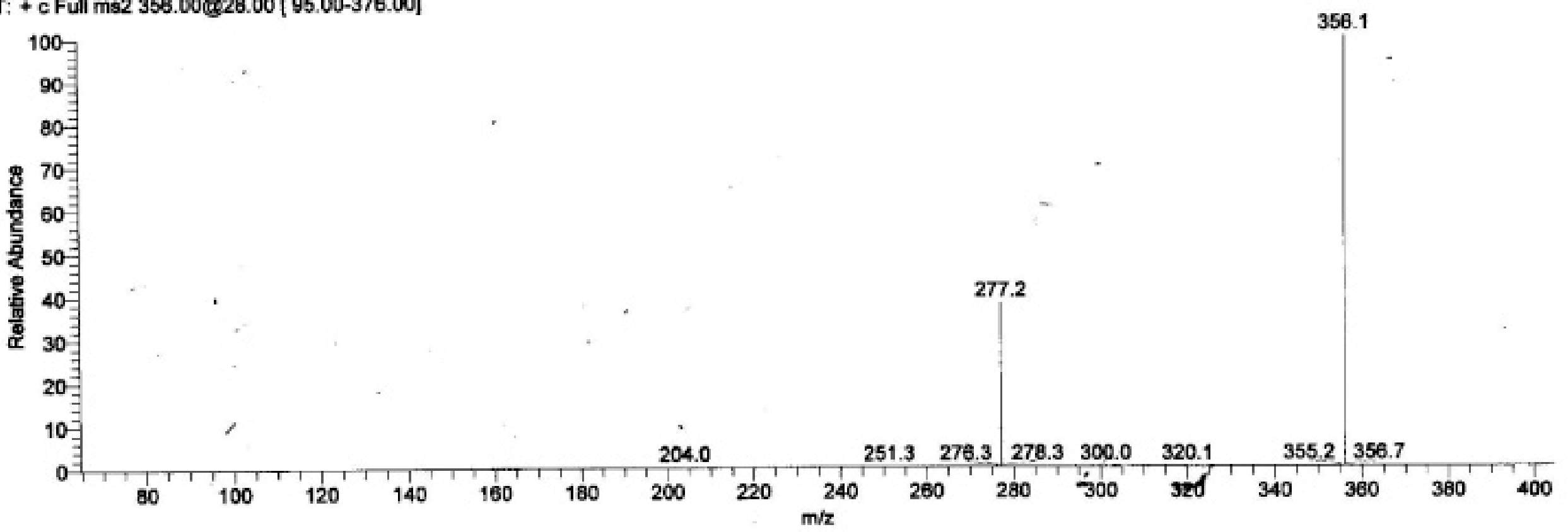
020429_03Aif_DOMARKAS_JDA36 "160-83 RT: 2.00-2.68 AV: 24 NL: 2.26E7 $\mathrm{T}:+\mathrm{c}$ Full $\mathrm{ms}$ [ $50.00-2000 . \overline{0} 0]$
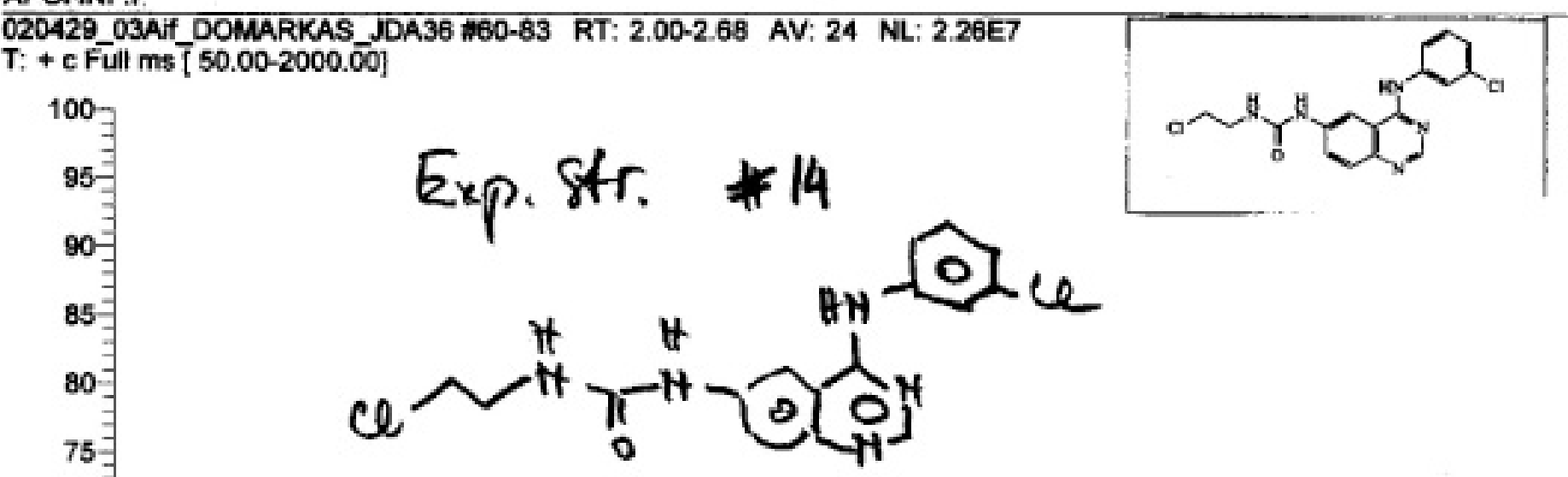

\subsection{8}

70
65

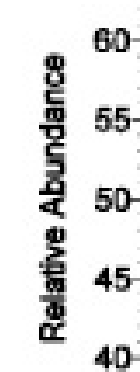

35

30

25

20

15

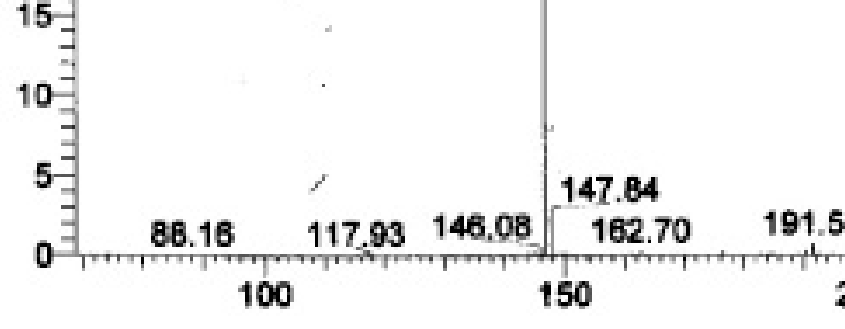

\subsection{9}

27.28 
D:Usersi...Id_27_04AIf_DOMARKAS_JDA40

\section{APCI}

030227_04Aif_DOMARKAS_JDA40 \#98-101 RT: 6.01-6.09 AV: 4 NL: 9.71E6

$\mathrm{T}$ : + c Füll ms [ 50.00-2000.00]

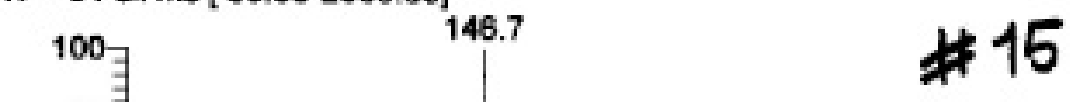

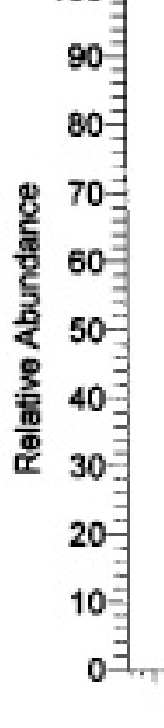

\section{7}

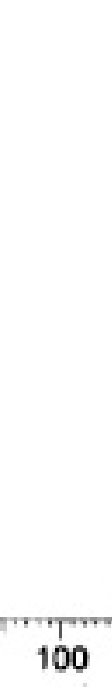

50

47.8

030227_04Aif_DOMARKAS_JDA40 \#84-93 RT: 5.84-5.95 AV: 10 NL: 9.85E6 $\mathrm{T}$ : + c Fül ms 2 420.00@28.00 [ 115.00-430.00]
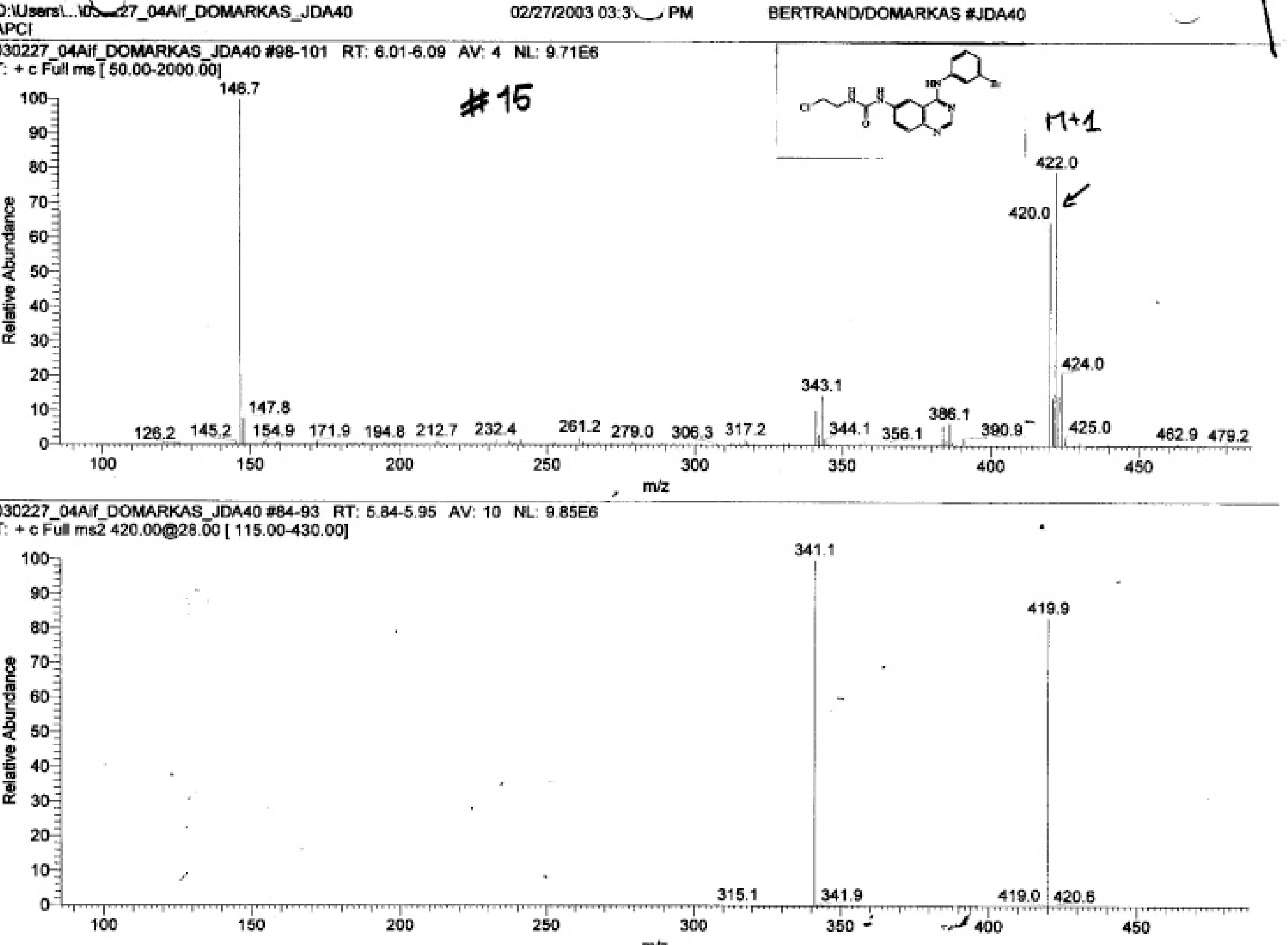

\section{$M+1$}

422.0

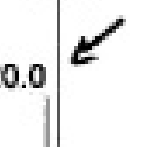

424.0

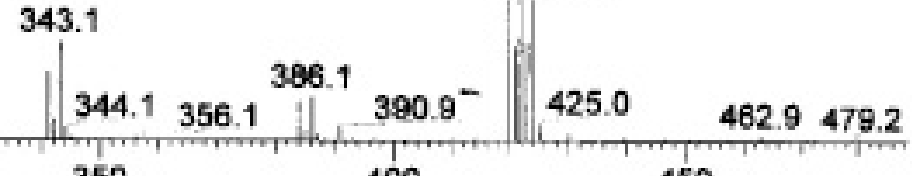
, $m / z$

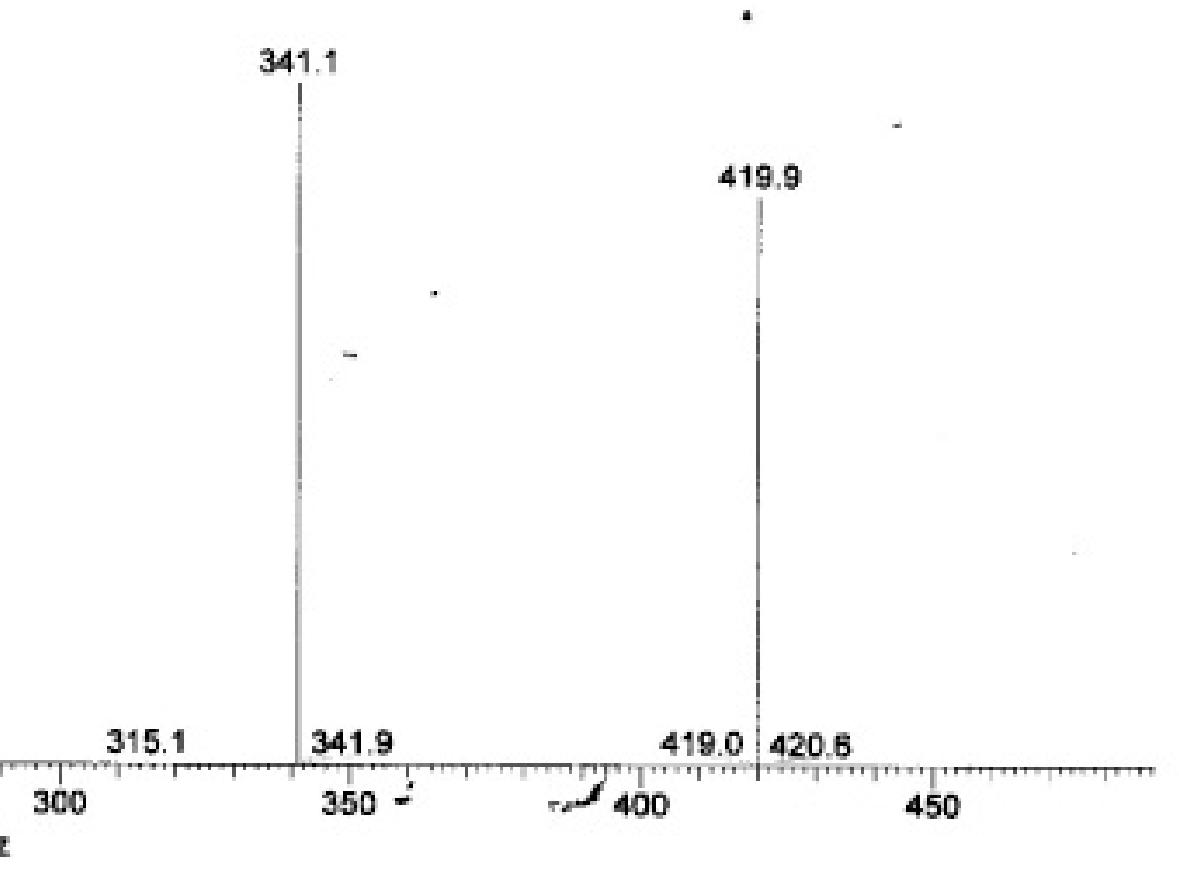




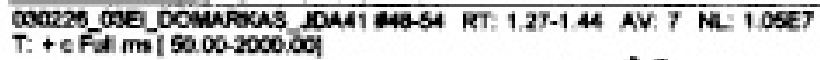

$\infty$

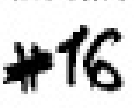

$00-3$

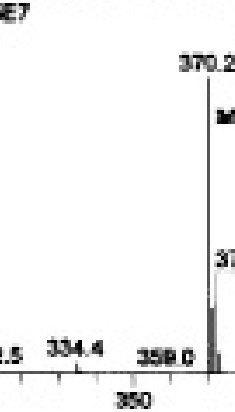

$r+1$

2

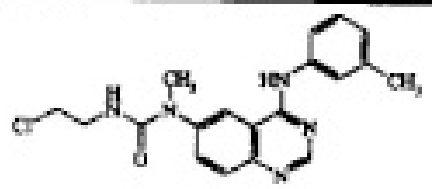

3722

3920

350

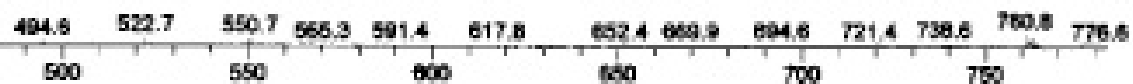

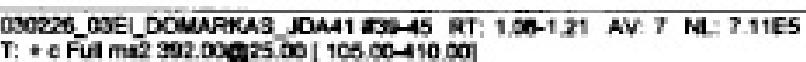

象 $00-100-1$
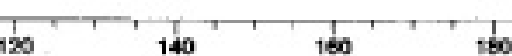

200

280

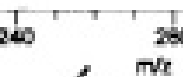

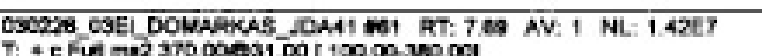

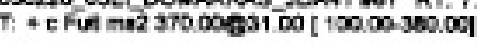

है $60-1$

, n
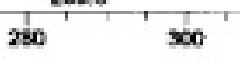

$\cos$

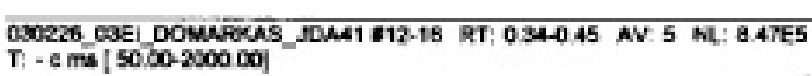

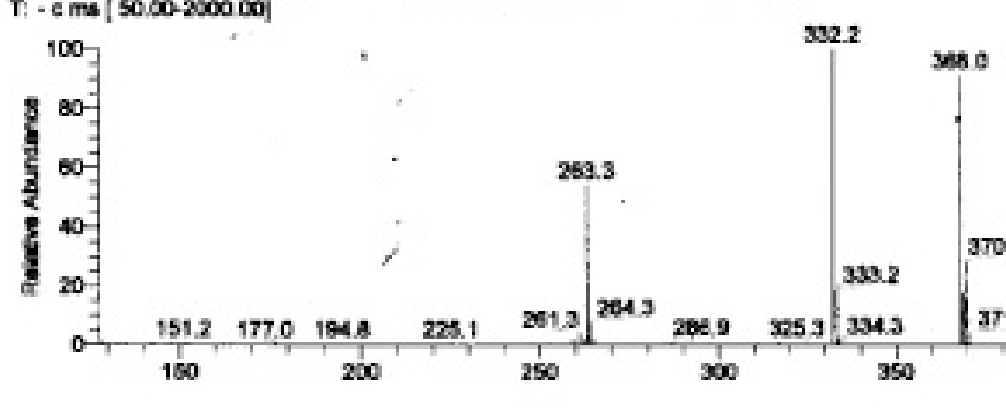

$\left.\left.\int_{3710}^{370.0}\right|_{400} ^{403.9}\right|_{1} ^{4059}$ $\frac{2323 \quad 260,2}{240}+24200$

$2 n 53$ 240
$\mathbf{m} 2$

220

$2000 \frac{200}{300}$

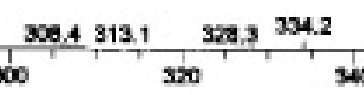

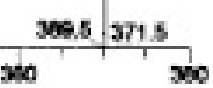

$450 \mathrm{~m} / \mathrm{s}$ 
030226_04EI_DOMARKAS JDA12 \#18-22 RT: $0.60-0.74$ AV: 5 NL: $1.71 E 7$

T: + c Fulli ms [ $50.00-2000.00]$

$100-$
$80-$
8
0

$\$ 17$

\section{2}
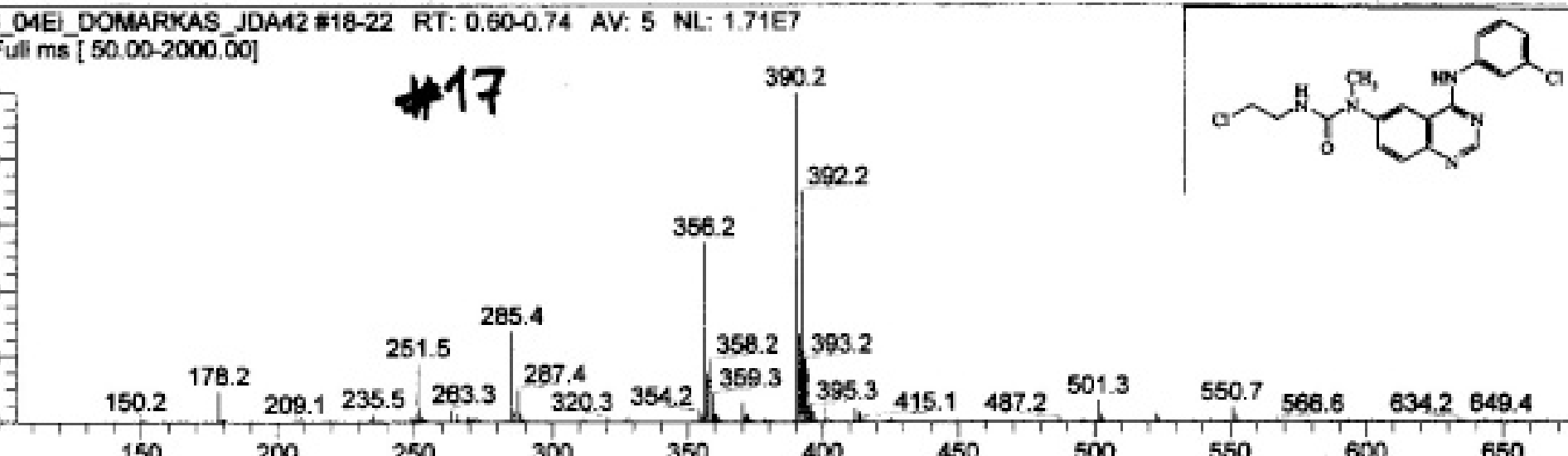

$\begin{array}{lll}209.1 & 235.5 & 263.3\end{array}$

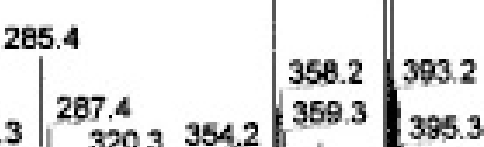

$385.3415 .1 \quad 487.2^{501.3}$

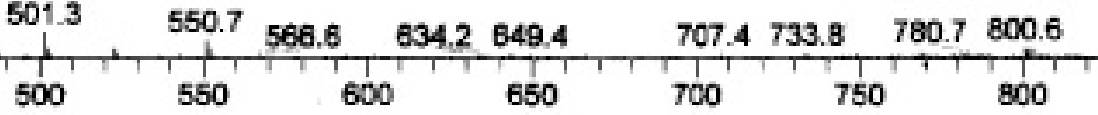

030226_04EI_DOMARKAS_JDA42 \#33-38 RT: 0.94-1.01 AV: 6 NL: 4.86E7

T: + c Full ms2 390.00@3 30.00 [ $105.00-400.00]$

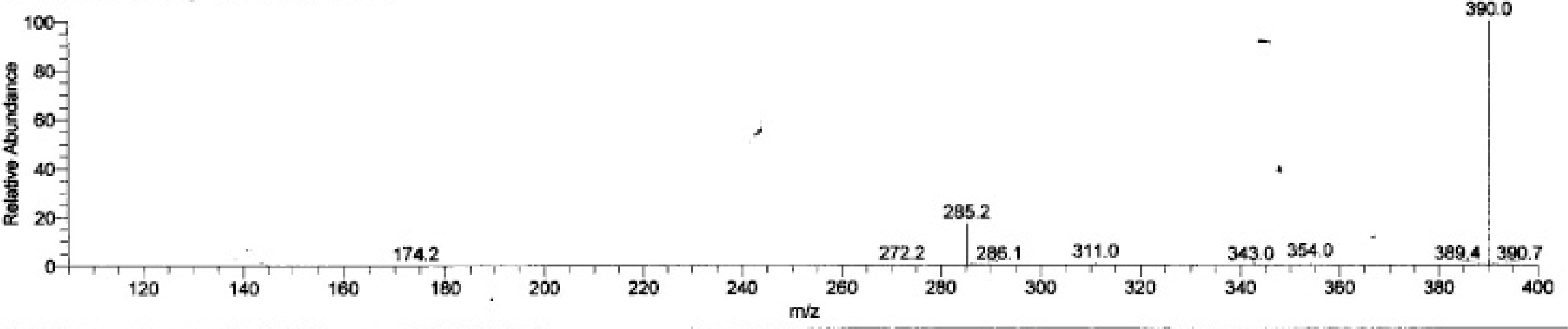

030226_04EL_DOMARKAS_JDA42 \#54-59 RT: 1.33-1.46 AV: 6 NL: 2.82E6

T: - c Full ms [ $50.00-2000.00]$
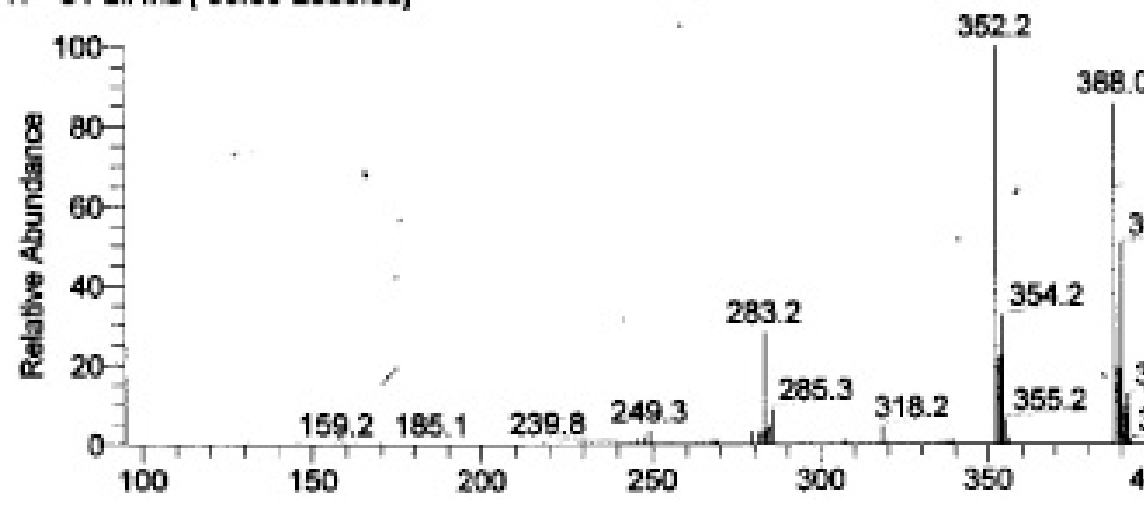

3900

391.9

$\begin{array}{llllllllllll}382.9 & 423.8 & 478.4 & 507.8 & 544.7 & 5807 & 603.5 & 633.7 & 686.5 & 7301 & 746.6 & 778.7\end{array}$

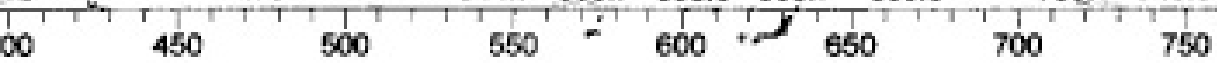




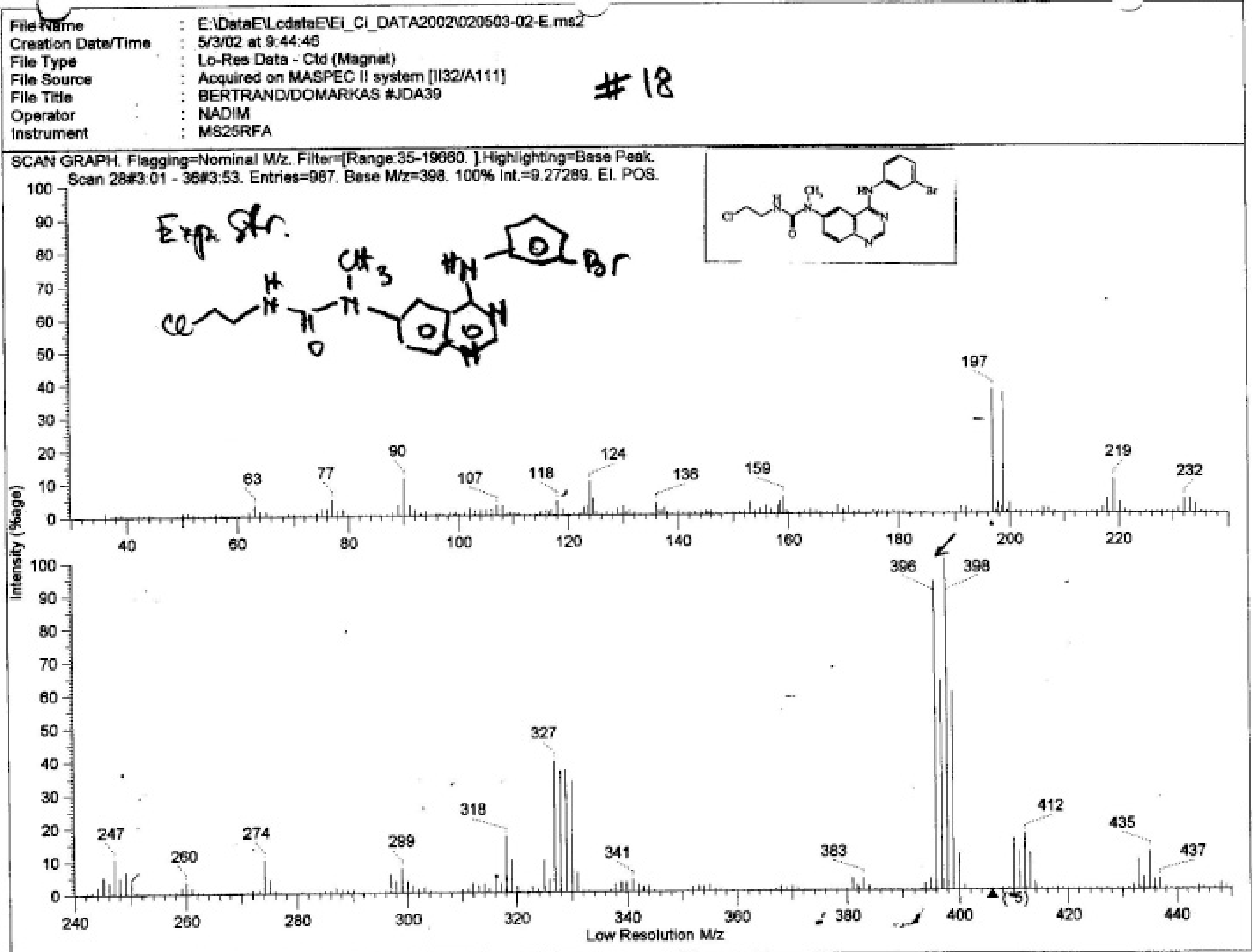


030312_02EI_DOMARKAS_JDA73 \#14-17 RY: 0.45-0.53 AV: A NL: 6.12E7

T: $4 \mathrm{cms}[50.00-2000 . \mathrm{co}]$
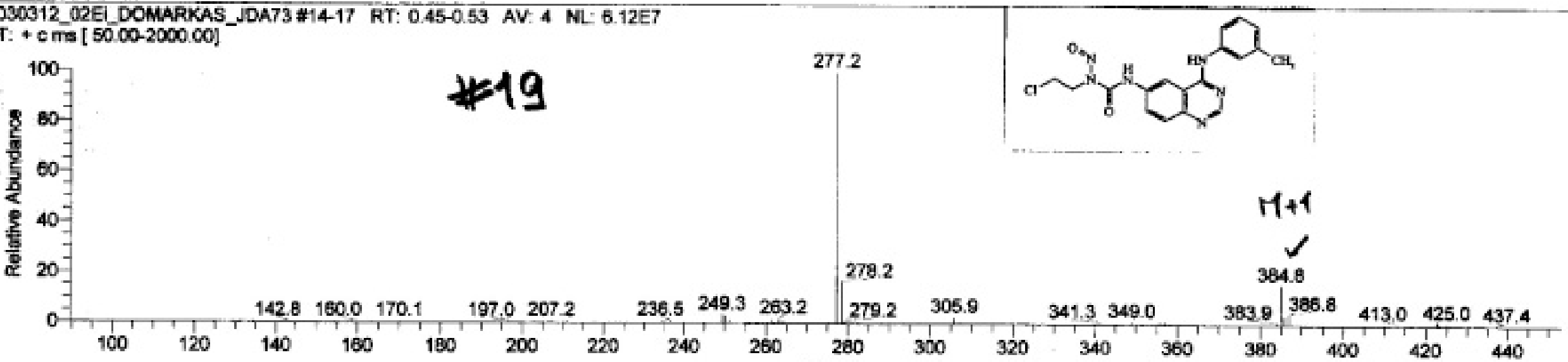

030312 02E1 DOMARKAS JDA73\#14-17 RT: $0.45-0.53$ AV: 4

$\mathrm{T}:+\mathrm{c}$ ms [ $50.00-200 \overline{0} .00]$

$\mathrm{m} / \mathrm{z}=89.7-452.9$

$\begin{array}{crr}\mathrm{m} / \mathrm{z} & \text { Intensity } & \text { Relative } \\ 249.3 & 1970536.3 & 3.22 \\ 250.2 & 1841170.5 & 3.01 \\ 277.2 & 61236264.0 & 100.00 \\ 278.2 & 10033336.0 & 16.38 \\ 384.8 & 9620522.5 & 15.71 \\ 385.8 & 1912317.0 & 3.12 \\ 386.8 & 2583000.3 & 4.22\end{array}$

\section{2_02Ei_DOMARKAS_JDA73 \#50-52 RT: 1.30-1.34 AV: 3 NL: 5.54E4}

T: + c Full ms2 $385.00827 .00[105.00-395.00]$

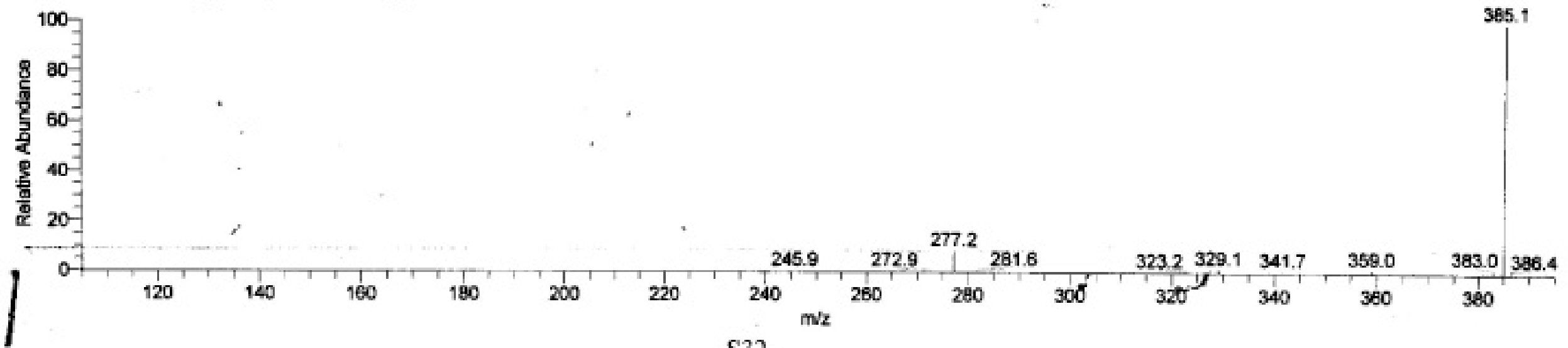


ESI

030325_07E_DOMARKAS_JDA65 26-28 RT: 0.75-0.81 AV: 3 NL: $1.57 E 7$

$\mathrm{T}:+\mathrm{cms}$ [ $5 \overline{0.00-2000.00]}$

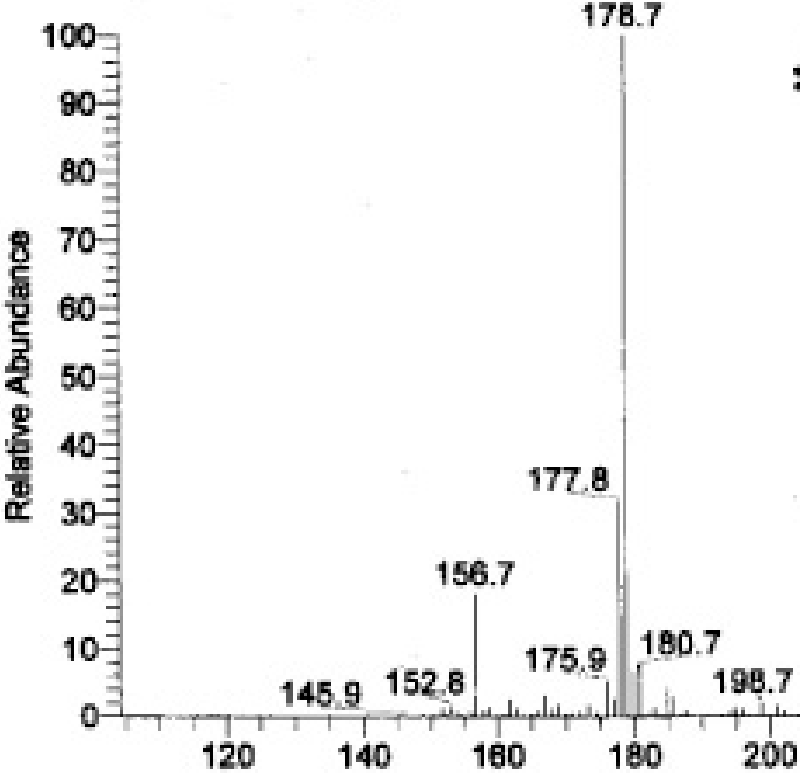

$\# 20$

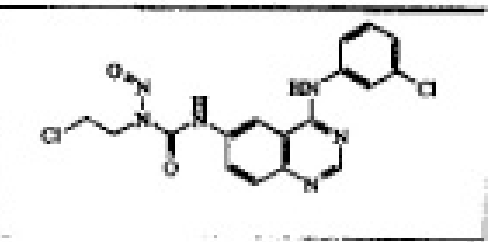

030325_07EI_DOMARKAS JDA65 \#34-36 RT: 0.95-0.99 AV: 3 NL: $3.20 E 5$

$\mathrm{T}:$ + c Füll ms2 405.00@25.00 [ 110.00-415.00]

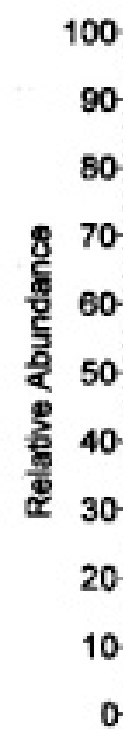

$100-30-3$

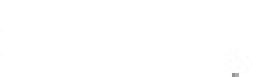


030311_07El_DOMARKAS_JDA66 \#5-7 RT: 0.15-0.21 AV: 3 NL: 2.84E7

$\mathrm{T}:+\mathrm{c}$ ms [ $50.00-2000.00]$

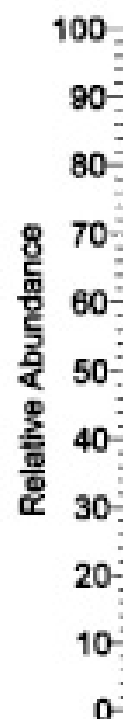

\#21

$90-$

30

70

$60-$

50

$40=$

$30=$

$20-$

$10 \mathrm{G}$

132.5149 .1

261.3

$150 \quad 200$

00
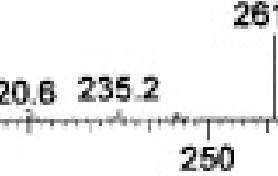

263.2

250

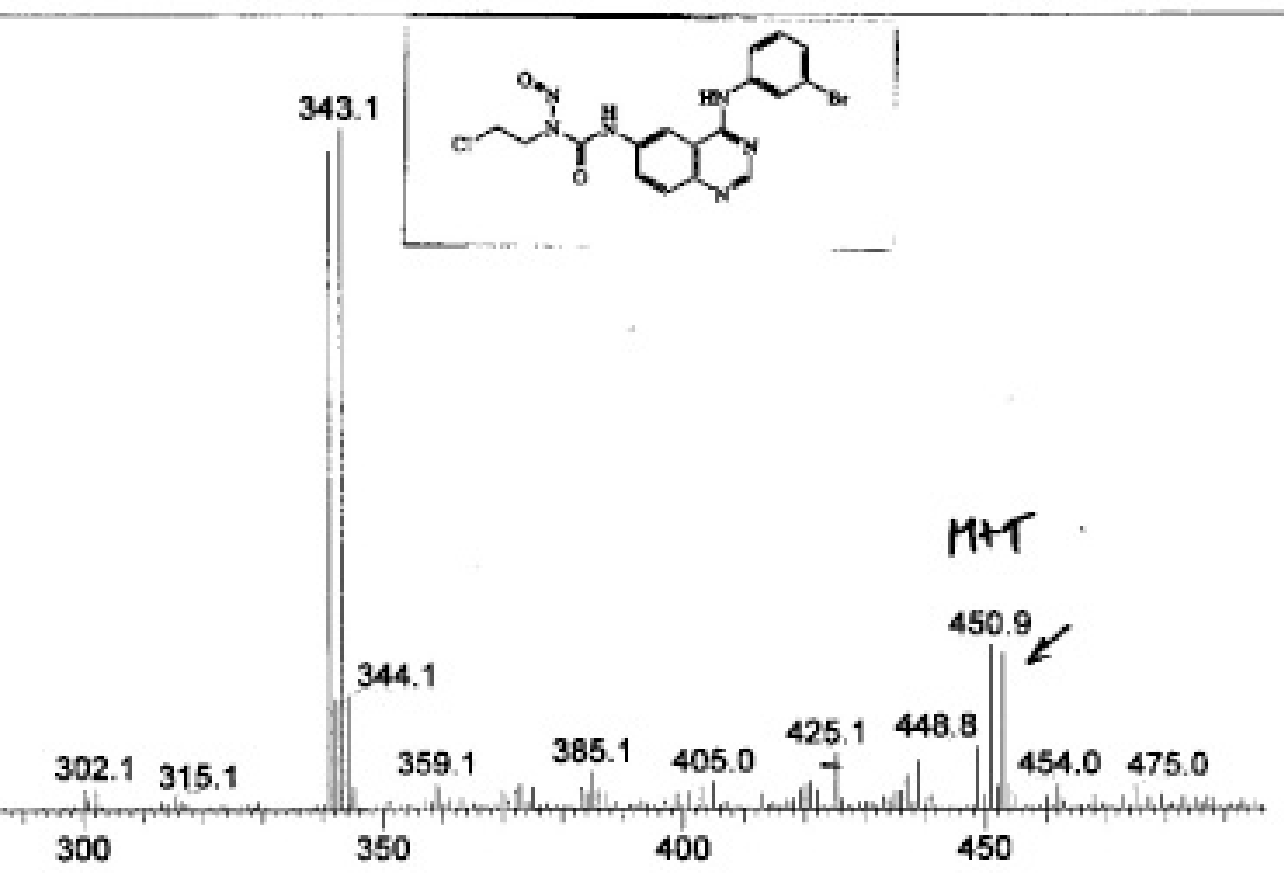

030311 07Ei_DOMARKAS JDA66\#5-7 RT: $0.15-0.21$ AV: 3

$\mathrm{T}:+\mathrm{c}$ ms [ $\overline{50} .00-200 \overline{0} .00]$

$\mathrm{m} / \mathrm{z}=106.4-496.8$

$\mathrm{m} / \mathrm{z}$

261.3

341,1

342.1

343.1

344.1

450.9

453.0

Intensity Relative

$3671818.3 \quad 12.47$

$28464248.0 \quad 96.67$

$4783479.0 \quad 16.25$

29443616.0100 .00

$4952343.3 \quad 16.82$

$7178803.0 \quad 24.38$

$6818951.7 \quad 23.16$ 
D:Userst..K A25_04El_DOMARKAS_JDA5B

ESI

03/25/2003 11:2-05 AM

BERTRANDIDOMARKAS *NDAS6

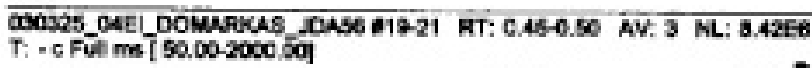

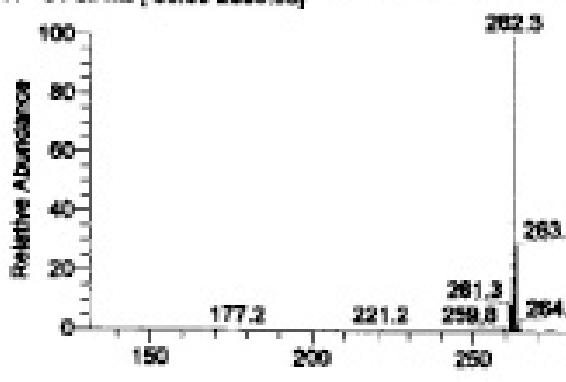

- 22 M-1<smiles>CCO[Te](C)(=O)OC</smiles>

$\mathrm{m} / \mathrm{z}$

$$
\begin{array}{cr}
\text { Intensity } & \text { Relative } \\
781268.3 & 9.28 \\
8415081.0 & 100.00 \\
2490768.0 & 29.60 \\
2135298.3 & 25.37 \\
542671.0 & 6.45 \\
863498.3 & 10.26 \\
555154.7 & 6.60
\end{array}
$$$$
261.3
$$$$
262.3
$$

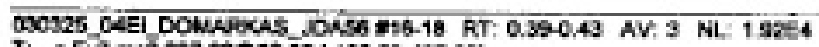
T: - c Ful maz $\$ 97,00025.00$ ? $105.00-407,00 \mid$

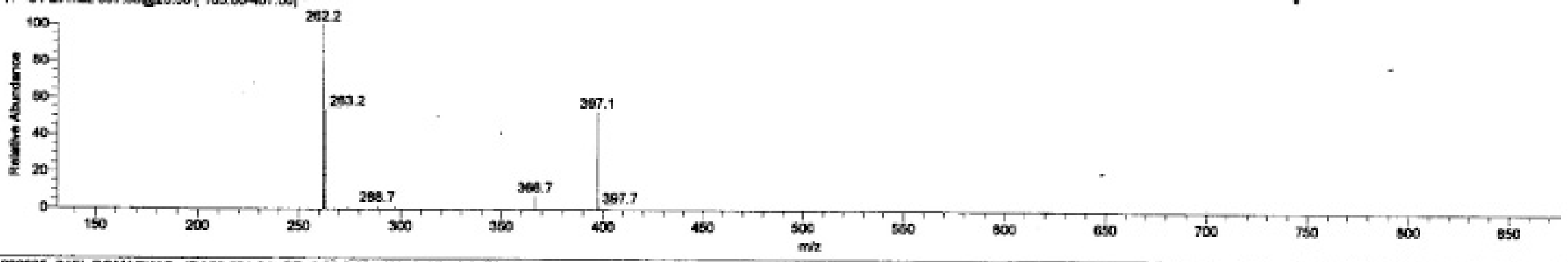

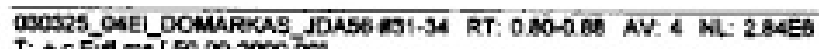
$\mathrm{T}:+0$ Full mi $[50.00-2000.0 \mathrm{~d})$

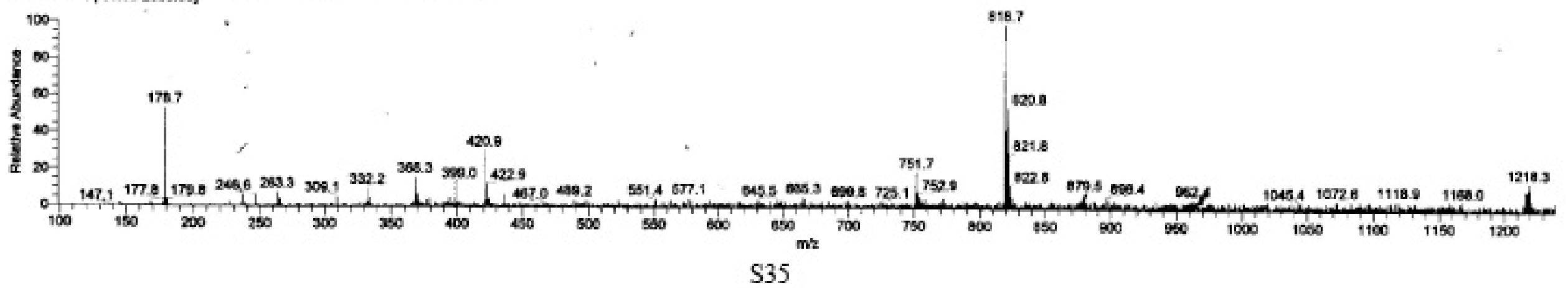


30325_03Ei_DOMARKAS_JDA58 \#8-11 RT: 0.20-0.28 AV: 4 NL: $1.54 E 7$

\section{T: $-\mathrm{cms}$ [ 50.00-2000.00]}

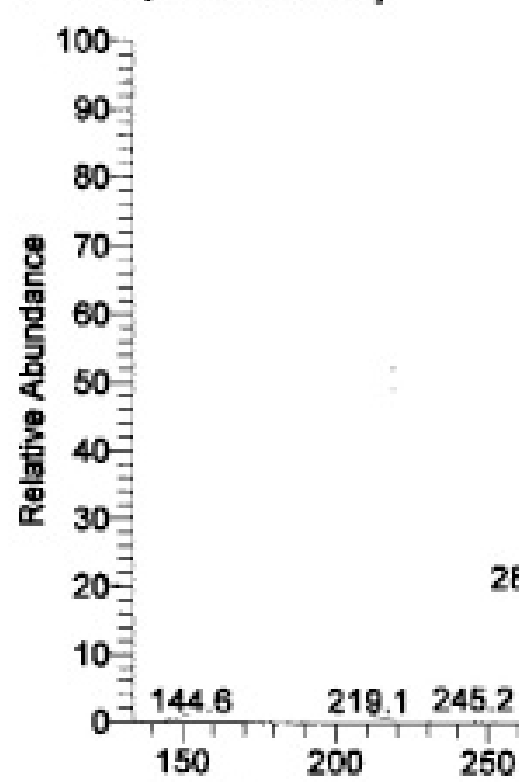

\section{2}

\#23

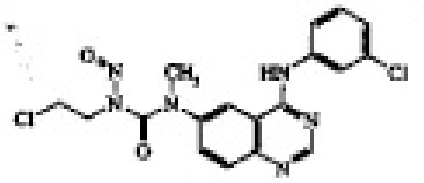

$150-200-250$

284.2<smiles>C[14CH3]</smiles>

281,2

285.2 311.8 389.9

030325_03Ei_DOMARKAS_JDA58 *25-28 RT: 0.59-0.65 AV: 4 NL: 5.73E4

$\mathrm{T}:$ - c Füll ms 2 417.00@21.00 [ $110.00-427.00]$

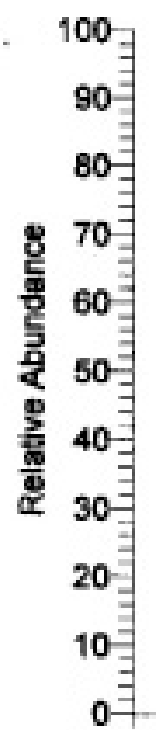

283.2 $\mathrm{m} / \mathrm{z}$

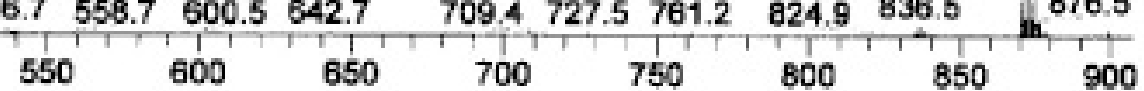

...
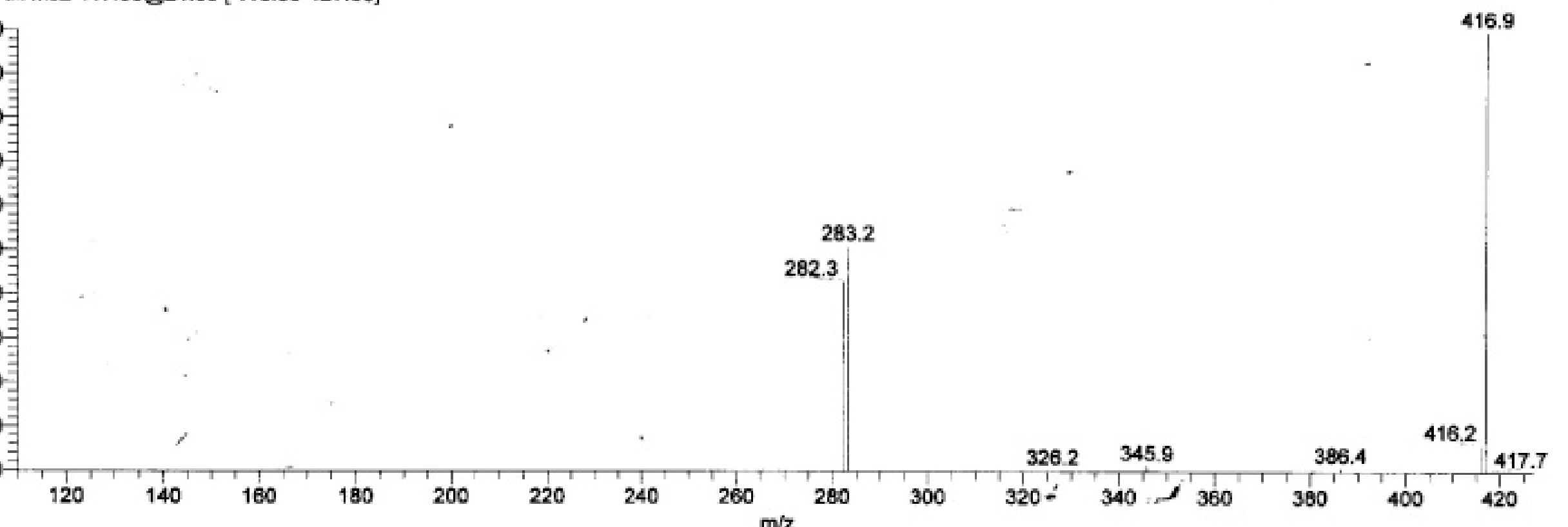


\section{APCI MEOH}

O20617 01AAT DOMARIKAS_JDA52 \#17-18 RT: 0.48-0.51 AV: 2 NL: 1.4967

$\mathrm{T}:+\mathrm{cms}$ [ $50.00-2000.00]$

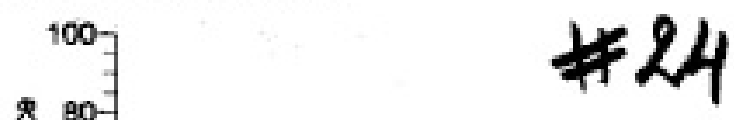

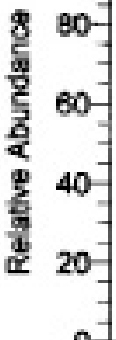

$\$ 24$

107

$\begin{array}{lllllllll}150.0 & 159.7 & 181.0 & 200.1 & 237.1 & 249.3 & 262.0 & 295.2 & 313.2\end{array}$

$200 \quad 250$
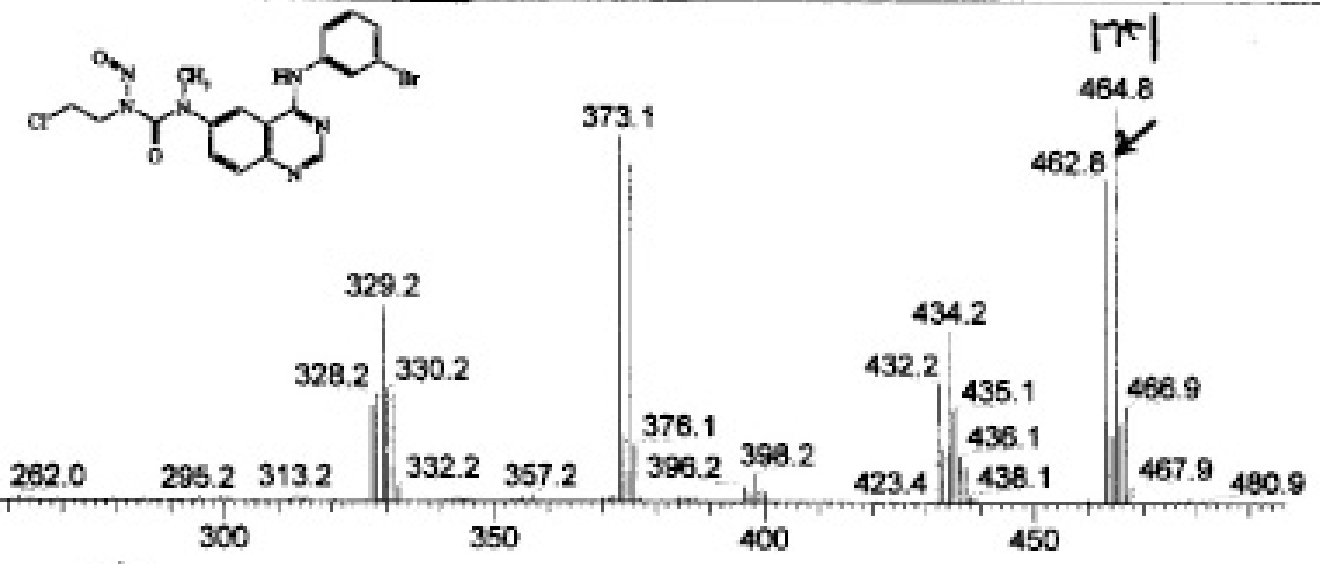

$\mathrm{m} / 2$

020617_01Aif_DOMARKAS_JDA52 $42-45$ RT: 0.840 .88 AV: 4 NL: 3.29E7

$\mathrm{T}:+c$ Full $m s 2$ 465.00@20.00 | $125.00-485.00$ )

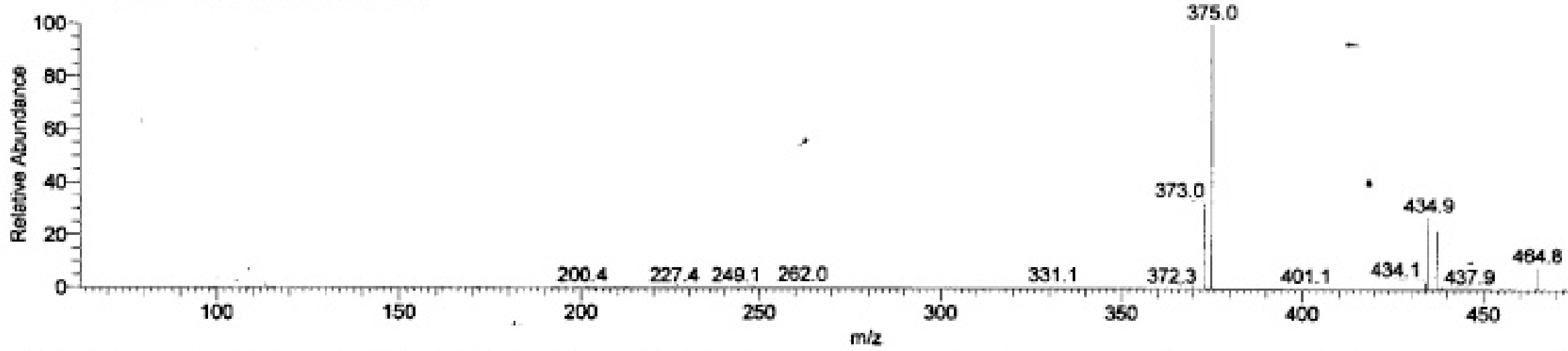

020617_01AVt_DOMARKAS_JDA52 "165-67 RT: 1.27-1.32 AV: 3 NL: $2.82 E 5$

T: - e Fưt ms [50.00-2000.00]

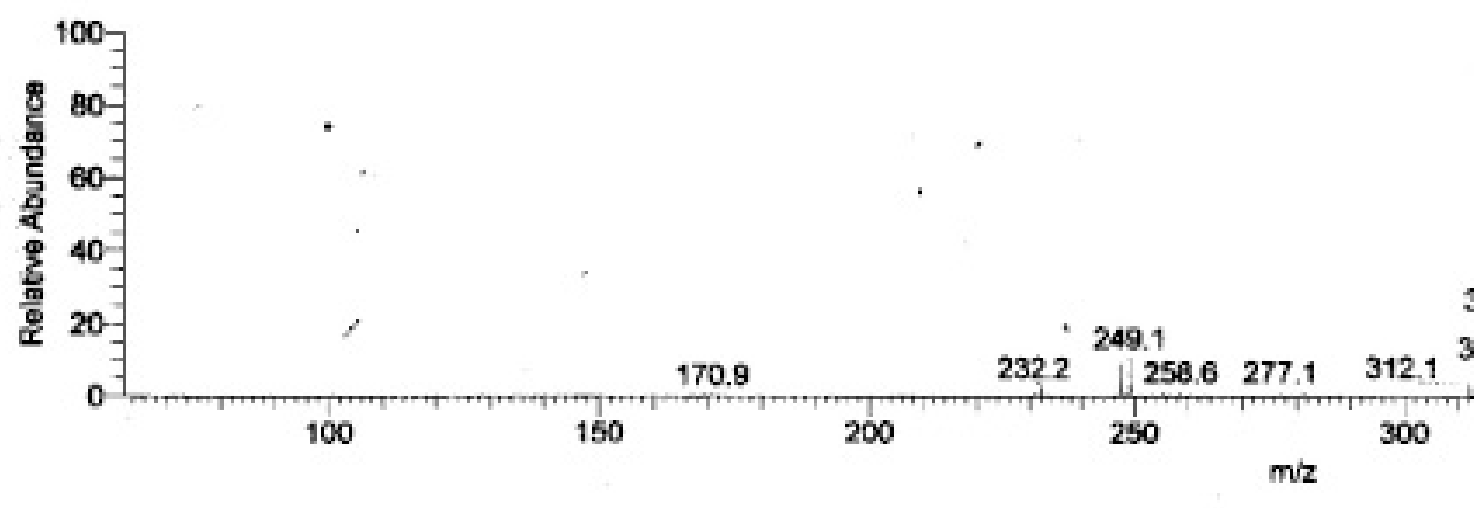




\section{Elemental Composition dis 13}

44

Ion: F.th Even and odd

Hetercatom Max: Linics:

354.127670

Nates

356.127670
10.9

\begin{tabular}{r}
$\mathbf{m D a}$ \\
-2.5 \\
-2.0 \\
-1.7 \\
-1.6 \\
-0.3 \\
-0.2 \\
0.1 \\
\hline 0.5 \\
2.0 \\
2.1 \\
2.5 \\
3.5
\end{tabular}
PPM Calc. Mass

$-7,1 \quad 356,125133$

$-5.8 \quad 356.125620$

$-4.7 \quad 356.125983$

$-4.4 \quad 356,126108$

$-1.356 .127326$

$-0.6 \quad 356.127458$

0.4356 .127813

$1.8 \quad 356.128300$

5.5356 .129643

$5.8 \quad 356.129726$

6.9356 .130130

$7,1 \quad 356,130213$ $\begin{array}{rrrrrrr}-4.2 & 0 & 0 & 3 & 0 & 0 & 0 \\ 32.9 & 200 & 400 & 7 & 4 & 3 & 1\end{array}$

$\begin{array}{lllllll}\text { DAB } & \mathrm{C} & \mathrm{H} & \mathrm{N} & \mathrm{O} & \mathrm{Cl} & \mathrm{Br}\end{array}$

$\begin{array}{llllll}7.0 & 15 & 21 & 4 & 4 & 1 \\ 2.5 & 12 & 24 & 5 & 3 & 2\end{array}$

$\begin{array}{llllll}16.5 & 19 & 14 & 5 & 3 & 2\end{array}$ $\begin{array}{rrrrrr}-2.0 & 9 & 27 & 6 & 2 & 3\end{array}$ $16.0 \quad 21 \quad 16$

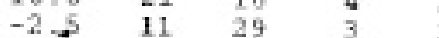

$\begin{array}{llll}-2 & 11 & 29 & 3\end{array}$

\begin{tabular}{rrrrrrr}
22.5 & 18 & 19 & 5 & 1 & 1 \\
\hline 7.6 & 15 & 22 & 6 & & 2 & \\
6.5 & 17 & 24 & 3 & 1 & 2 & \\
0.5 & 11 & 27 & 5 & 3 & & 1 \\
2.0 & 14 & 27 & 4 & & 3 & \\
-4.0 & 8 & 30 & 6 & 2 & 1 & 1
\end{tabular}

-1 - 


\begin{tabular}{|c|c|c|c|c|c|c|c|c|c|c|}
\hline $\begin{array}{l}\text { Heteroatom } \\
\text { Limits }\end{array}$ & 44 & Ion: & oth Even and & Odd & & & & & & \\
\hline 376,073120 & & 7.4 & & $\begin{array}{r}-4.2 \\
39.9\end{array}$ & $\begin{array}{r}0 \\
200\end{array}$ & $400^{\circ}$ & $\begin{array}{l}3 \\
7\end{array}$ & $\begin{array}{l}0 \\
4\end{array}$ & $\begin{array}{l}0 \\
3\end{array}$ & 1 \\
\hline Mass & mDa. & $\mathrm{PPM}$ & Calc. Mass & DBE & c & H & $\mathbf{N}$ & 0 & $\mathrm{cl}$ & Br \\
\hline 376.073120 & $\begin{array}{r}-2.6 \\
-2.2 \\
-2.1 \\
-1.8 \\
-0.9 \\
-0.4 \\
0.1 \\
\end{array}$ & $\begin{array}{l}-6.9 \\
-6.0 \\
-5.6 \\
-4.7 \\
-2.4 \\
-1.1 \\
0.2 \\
\end{array}$ & $\begin{array}{l}376.070511 \\
376.070874 \\
376.070998 \\
376.071361 \\
376.072216 \\
376.072704 \\
376,073191\end{array}$ & $\begin{array}{r}7.0 \\
21.0 \\
2.5 \\
14.5 \\
20.5 \\
1.0 \\
11.5\end{array}$ & $\begin{array}{l}14 \\
21 \\
11 \\
18 \\
23 \\
20 \\
17 \\
\end{array}$ & $\begin{array}{r}18 \\
8 \\
31 \\
11 \\
10 \\
13 \\
116 \\
\end{array}$ & $\begin{array}{l}4 \\
6 \\
5 \\
7 \\
3 \\
4 \\
5 \\
5\end{array}$ & $\begin{array}{l}4 \\
2 \\
3 \\
1 \\
3 \\
2 \\
1 \\
\end{array}$ & $\begin{array}{l}3 \\
1\end{array}$ & \\
\hline & 0.5 & $\begin{array}{l}0.4 \\
1.5\end{array}$ & $\begin{array}{l}375.073278 \\
376.073678\end{array}$ & $\begin{array}{l}5.5 \\
7.0\end{array}$ & 11 & 79 & 7 & 3 & 3 & \\
\hline & 1.5 & 4.0 & 376.074617 & 5.0 & $\begin{array}{l}14 \\
13\end{array}$ & $\begin{array}{l}19 \\
.21\end{array}$ & $\begin{array}{l}6 \\
4\end{array}$ & 4 & & \\
\hline & 1.8 & 4.7 & 376.074856 & 25.0 & 26 & 3 & 4 & & & 1 \\
\hline & 1.9 & 5.1 & 376.075021 & 6.5 & 16 & 21 & 3 & 1 & 3 & \\
\hline & 2,0 & 5.3 & 376.075104 & 0.5 & 10 & 26 & 5 & 3 & 1 & \\
\hline & 2.5 & 6.6 & 376.075591 & -4.0 & 7 & 27 & 6 & 2 & 2 & \\
\hline
\end{tabular}


Heteroatom Max: Linits :

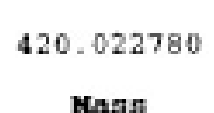

420.022780
44 Ion: Eoch Even and odd

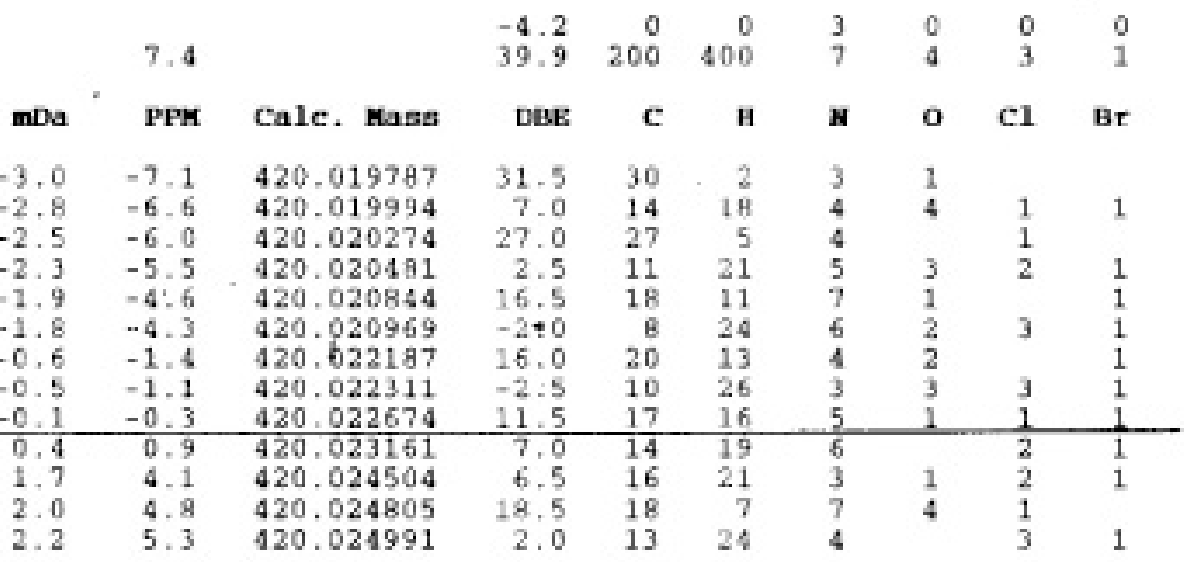

\section{7}

$\because$ 
Heteroatom Kax: is Ion: Futh Even and coid

Limits :

376.143450

Mass

370.143450

\begin{tabular}{|c|c|c|c|c|c|c|c|c|c|}
\hline & & & $-4,2$ & 0 & D & 3 & 0 & 0 & 0 \\
\hline & 7.4 & & 39.9 & 200 & 400 & 7 & 4 & 3 & 1 \\
\hline mDa. & PPM & Cale. Mass & DEE & c & H & $\boldsymbol{M}$ & o & cl & Br \\
\hline-2.7 & -7.2 & 370.140783 & 7.0 & 16 & 23 & 4 & 4 & 1 & \\
\hline-2.2 & -5.9 & 370.141270 & 2.5 & 13 & 26 & 5 & 3 & 2 & \\
\hline-1.8 & $-4,9$ & 370.141633 & 16.5 & 20 & 16 & 7 & 1 & & \\
\hline-1.7 & -4.6 & 370.141758 & -20 & 10 & 39 & 6 & 2 & 3 & \\
\hline-0.5 & -1.3 & 370.142976 & 16.0 & 22 & is & 4 & 2 & & \\
\hline$-9,3$ & -0.9 & 370.143100 & -2.5 & 12 & 31 & 3 & 3 & 3 & \\
\hline 9.0 & 0.12 & 370.143463 & $11^{4} 5$ & 19 & 31 & 5 & 1. & 1 & \\
\hline 0.5 & 1.4 & $370+143950$ & -7.0 & 16 & 24 & 6 & & 2 & \\
\hline 1.8 & 5.0 & 370.145293 & 0.5 & 18 & 26 & 3 & 1 & 2 & \\
\hline 1.9 & 5.2 & 370.145376 & 0.5 & 12 & 29 & 5 & 3 & & \\
\hline 3,3 & 6.3 & 370.145780 & 2.0 & 15 & 29 & 4 & & 3 & \\
\hline 2.4 & 6.5 & 370.145863 & -4.0 & 9 & 32 & 6 & 2 & 1 & \\
\hline
\end{tabular}

,

$\because$ 


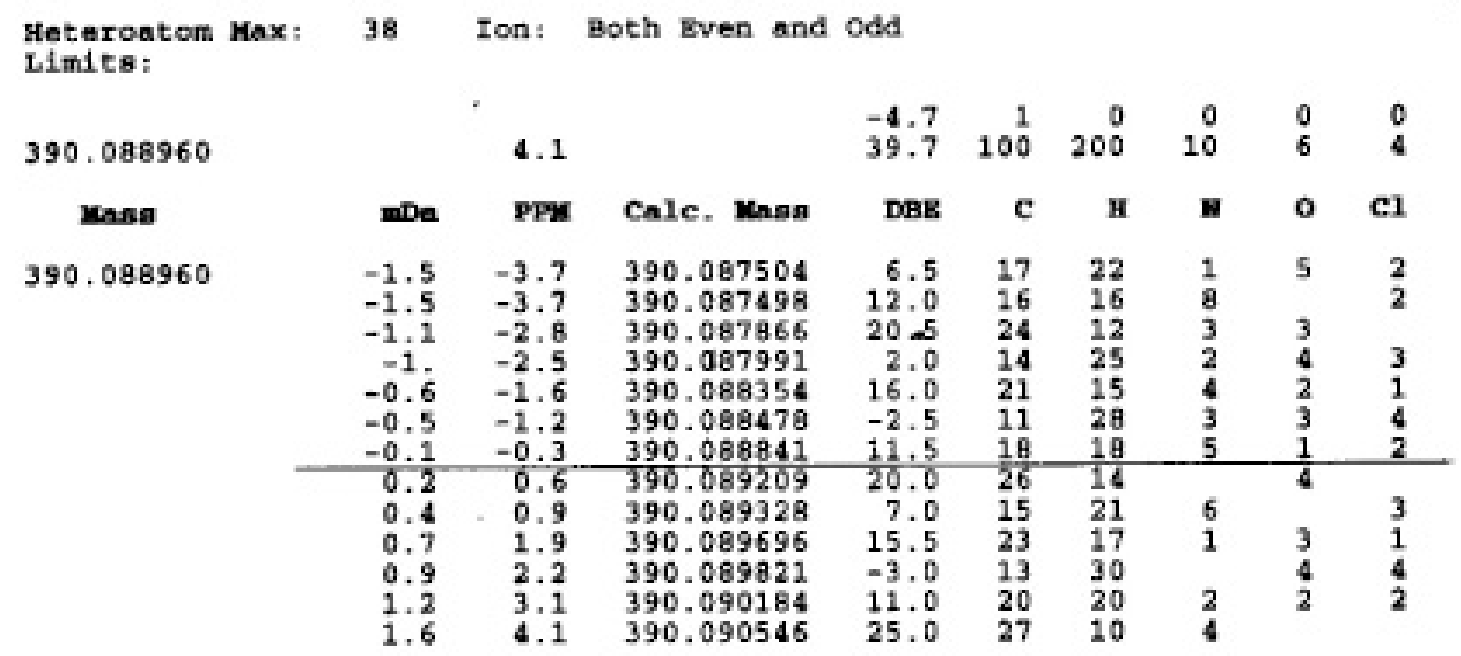


Heteroatan Max:

Limita :

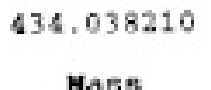

434.038210
Ion: Both Even and odd

\begin{tabular}{|c|c|c|c|c|c|c|c|c|c|}
\hline & 7.4 & & $\begin{array}{l}-6.2 \\
39.9\end{array}$ & 200 & $\begin{array}{r}0 \\
400\end{array}$ & $\begin{array}{l}3 \\
7\end{array}$ & $\begin{array}{l}0 \\
4\end{array}$ & $\begin{array}{l}0 \\
3\end{array}$ & $\begin{array}{l}0 \\
1\end{array}$ \\
\hline nDa & $=\quad \mathbf{P P H}$ & Calc. Masa & DBE & $=C$ & H & $\mathbf{N}$ & 0 & $\mathrm{Cl}$ & Br \\
\hline-2.8 & $-6 \cdot 4$ & 434.035437 & 31.5 & 31 & 4 & 3 & 1 & & \\
\hline-3.6 & -5.9 & 434.035644 & 7.0 & 15 & in & 4 & 4 & 1 & 1 \\
\hline$-2 \cdot 3$ & $-5 \cdot 3$ & 434.035924 & 37.0 & 23 & 7 & 4 & & 1 & \\
\hline-2.1 & -4.8 & 434.036131 & 2.5 & 13 & $\therefore 3$ & 5 & 3 & 2 & 1 \\
\hline-1.7 & -6.0 & 434.036490 & 16,5 & 19 & .3 & 7 & 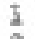 & & 1 \\
\hline$-1-6$ & -3.7 & 434.036619 & -2.0 & 9 & 36 & 6 & 2 & 3 & 1 \\
\hline$-0,4$ & -0.9 & 434.937837 & $16 \%$ & 21 & 15 & 4 & 2 & & 1 \\
\hline-0.2 & -0.6 & 434.037961 & -2.5 & 11 & 28 & 3 & 3 & 3 & 1 \\
\hline$\frac{0.1}{0.6}$ & $\begin{array}{r}0.3 \\
\end{array}$ & 434.038324 & 21.5 & 18 & 18 & $\frac{5}{8}$ & 1 & 1 & 1 \\
\hline $\begin{array}{l}0.6 \\
1.9\end{array}$ & $1-4$ & 434.056811 & 7.0 & 15 & 21 & 6 & & 2 & 1 \\
\hline 2.9 & $5-3$ & 934.060134 & $6 \cdot 3$ & 17 & 23 & 3 & 1 & 2 & 1 \\
\hline 2.4 & 56 & 434.040641 & $\begin{array}{r}18-3 \\
2.0\end{array}$ & $\begin{array}{l}19 \\
14\end{array}$ & 26 & 4 & 4 & $\frac{1}{3}$ & 1 \\
\hline
\end{tabular}


Hetereatom Max; L. $2 \mathrm{mits}$

385.118090

Mass

385,118090
44 Ion:
Elemental compositions?/8

Date : 2-AUC-2003

\begin{tabular}{|c|c|c|c|c|c|c|c|c|c|}
\hline & 7.4 & & $\begin{array}{l}-4.2 \\
39.9\end{array}$ & $\begin{array}{r}0 \\
200\end{array}$ & $\begin{array}{r}0 \\
400\end{array}$ & $\begin{array}{l}3 \\
7\end{array}$ & $\begin{array}{l}0 \\
4\end{array}$ & $\begin{array}{l}0 \\
3\end{array}$ & $\begin{array}{l}0 \\
1\end{array}$ \\
\hline mDa. & PPM & Calc. Maena & DAE $^{2}$ & c & H & $\boldsymbol{N}$ & 0 & Cl & $\mathrm{Br}$ \\
\hline $\begin{array}{l}-2.7 \\
-2.3 \\
-1.8 \\
-0.6 \\
-0.5 \\
-0.2 \\
\end{array}$ & $\begin{array}{l}-7,1 \\
-6,0 \\
-4.7 \\
-1.6 \\
-1.2 \\
-0,3\end{array}$ & $\begin{array}{l}385.115359 \\
385.115784 \\
385.116271 \\
385,127490 \\
385.117624 \\
385.137977\end{array}$ & $\begin{array}{r}11.0 \\
3.5 \\
-1.0 \\
17,0 \\
-1.5 \\
12.5 \\
\end{array}$ & $\begin{array}{r}20 \\
12 \\
9 \\
21 \\
11 \\
19 \\
\end{array}$ & $\begin{array}{l}24 \\
23 \\
26 \\
15 \\
28 \\
18 \\
\end{array}$ & $\begin{array}{l}3 \\
6 \\
7 \\
5 \\
4 \\
6\end{array}$ & $\begin{array}{l}4 \\
3 \\
3 \\
4 \\
2 \\
\end{array}$ & $\begin{array}{l}2 \\
3 \\
3 \\
1 \\
\end{array}$ & 1 \\
\hline $\begin{array}{l}0.4 \\
1.2 \\
1.7 \\
1.8 \\
2.1 \\
2.2 \\
2.3\end{array}$ & $\begin{array}{l}3.2 \\
4.5 \\
6.7 \\
5.4 \\
5.7 \\
5.9\end{array}$ & $\begin{array}{l}385,118464 \\
385.119319 \\
385.119807 \\
385.119894 \\
385.120170 \\
385.120294 \\
385.120377\end{array}$ & $\begin{array}{r}8.0 \\
12.0 \\
7.5 \\
1.5 \\
21.5 \\
3.0 \\
-3.0\end{array}$ & $\begin{array}{r}13 \\
20 \\
17 \\
11 \\
24 \\
14 \\
8\end{array}$ & $\begin{array}{l}21 \\
20 \\
23 \\
26 \\
13 \\
26 \\
29\end{array}$ & $\begin{array}{l}7 \\
3 \\
4 \\
6 \\
6 \\
5 \\
7\end{array}$ & $\begin{array}{l}1 \\
3 \\
2 \\
4\end{array}$ & $\begin{array}{l}2 \\
1 \\
2\end{array}$ & 1 \\
\hline
\end{tabular}


Heteratom Max: 44 Ion: Eoth Even and odd I.inies:

405.663230 7.4

Mass

405.063230

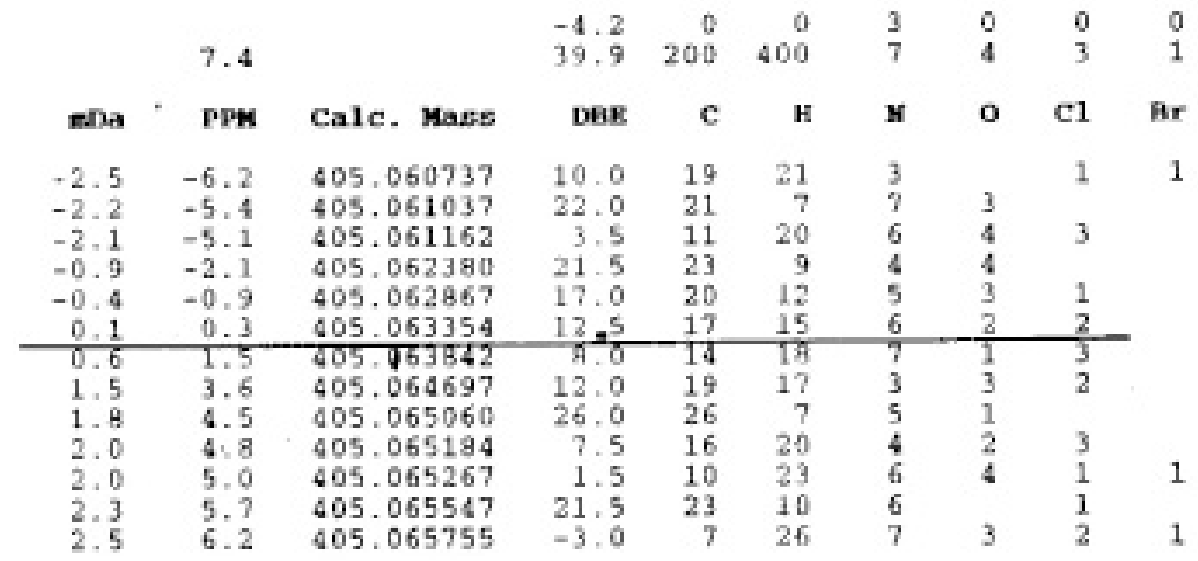


Hetercatom Max: 44 Ion: Euth Even and odd

Limitz:

449.012890

Mas:s

449.012890

\begin{tabular}{|c|c|c|c|c|c|c|c|c|c|}
\hline & $? .4$ & & $\begin{array}{r}-4.2 \\
39.9\end{array}$ & $\begin{array}{r}0 \\
200\end{array}$ & $\begin{array}{r}0 \\
400\end{array}$ & $\frac{3}{7}$ & $\begin{array}{l}0 \\
4\end{array}$ & $\begin{array}{l}0 \\
3\end{array}$ & $\begin{array}{l}0 \\
1\end{array}$ \\
\hline nDa & PPM & Calc. Mana & DBE & C. & H & $\mathbf{M}$ & 0 & C: & Br \\
\hline-2.9 & -6.5 & 449.009950 & .12 .5 & 30 & 1 & 4 & 2 & & \\
\hline-2 - คู & -6.3 & 449.010075 & 14.0 & 20 & 14 & 3 & 3 & 3 & \\
\hline-7.5 & -5.5 & 449.010439 & 28.0 & 27 & 4 & 5 & 1 & 1 & \\
\hline$=2.2$ & -5.0 & 449.010645 & 3.5 & 11 & $3 n$ & 6 & 4 & 2 & 1 \\
\hline-2.0 & -4.4 & 469.010925 & 23.5 & 24 & $?$ & 6 & & 2 & \\
\hline$-1,8$ & -3.9 & 449.011132 & -2.0 & B & 23 & 7 & 3 & 3 & 1 \\
\hline-0.6 & -1.4 & 449.012267 & 23.0 & 26 & 9 & 3 & 1 & 2 & \\
\hline$-0,5$ & $=1.2$ & 449.012351 & 17.0 & 20 & 12 & 5 & 3 & & 1 \\
\hline$=0,4$ & -0.9 & 449.012475 & -1.5 & 10 & 25 & 4 & 4 & 3 & 1 \\
\hline-0.1 & -0.3 & 449,012755 & 18.5 & 23 & 12 & 4 & & 3 & \\
\hline-0.1 & $-0,1$ & $469.012 \mathrm{B3} \mathrm{B}$ & 12.5 & 17 & 15 & 6 & 2 & 1 & 1 \\
\hline $0 . \overline{4}$ & 1. & 449.013325 & 8.0 & 14 & 18 & 7 & 1 & 2 & 1 \\
\hline 1.3 & 2.9 & 449.014180 & 12,0 & 19 & 17 & 3 & 3 & 1 & 1 \\
\hline 1. 8 & 4.0 & $449.01466 \mathrm{~B}$ & 7.5 & 16 & 20 & 4 & 2 & 2 & 1 \\
\hline 2,1 & 4,8 & 449.015031 & 21.5 & 23 & 16 & 6 & & & 1 \\
\hline 2,3 & 5.0 & 449.015155 & 3.0 & 13 & 23 & 5 & 1 & 3 & 1 \\
\hline
\end{tabular}

$\checkmark$ 
Heteroatom Mas: 44 Ion: Foch Even and odd

\section{Limits:}

399.133550

Mass

399.133550

\begin{tabular}{|c|c|c|c|c|c|c|c|c|}
\hline & 7,4 & & $\begin{array}{l}-4.2 \\
39.9\end{array}$ & 200 & $\begin{array}{r}0 \\
400\end{array}$ & $\begin{array}{l}3 \\
7\end{array}$ & $\begin{array}{l}0 \\
4\end{array}$ & $\begin{array}{l}0 \\
3\end{array}$ \\
\hline$m b a$ & PPH & Calc. Mansa & DAE & C & н & $\mathbf{N}$ & o & $\mathrm{C} 1$ \\
\hline-2.5 & -6.4 & 399.131009 & 10.0 & 21 & 26 & 3 & & \\
\hline-2.1 & -5.3 & 399.131434 & 3.5 & 13 & 25 & 6 & 4 & 2 \\
\hline-1.6 & -4.1 & 399.131921 & $-1,0$ & 10 & $2 R$ & 7 & 3 & 3 \\
\hline-0.4 & -1.0 & 399.133140 & 17.0 & 22 & 17 & 5 & 3 & \\
\hline$-0,3$ & -0.7 & 399.133264 & -1.5 & 12 & 30 & 4 & 4 & 3 \\
\hline 0.1 & 0.2 & $399-133627$ & $1 \approx 5$ & 12 & 20 & 6 & 2 & 1 \\
\hline 0.6 & 1.4 & 399.134114 & H.0 & 16 & 23 & 7 & 1 & 2 \\
\hline 1.4 & $3-6$ & 399.134970 & 12.0 & 21 & 22 & 3 & 3 & 1 \\
\hline 1.9 & 4.8 & 399.135457 & 7.5 & 18 & 25 & 4 & 2 & 2 \\
\hline 2,0 & 5.0 & 399.135540 & 1.5 & 12 & 28 & 6 & 4 & \\
\hline 2,3 & 5.7 & 399.135820 & 21.5 & 25 & 15 & 6 & & \\
\hline 2.4 & 6.0 & 399,135964 & 3.0 & 15 & $2 B$ & 5 & 1 & 3 \\
\hline 2.5 & 6.2 & 399.136027 & $-3,0$ & 9 & 31 & 7 & 3 & 1 \\
\hline
\end{tabular}

1 
Heteroatom Kax: Limits:

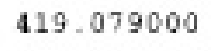

Masa

419.079000
44

Ion: Both Even and odd

\begin{tabular}{|c|c|c|c|c|c|c|c|c|}
\hline & 7.4 & & $\begin{array}{l}=4.2 \\
39.9\end{array}$ & $\begin{array}{r}0 \\
200\end{array}$ & $\begin{array}{r}10 \\
400\end{array}$ & $\begin{array}{l}3 \\
7\end{array}$ & $\begin{array}{l}0 \\
4\end{array}$ & $\begin{array}{l}0 \\
3\end{array}$ \\
\hline mon & PPM & Cale. Mass & DRE & c & H & $\mathbf{N}$ & $\mathrm{o}$ & Cl \\
\hline-2.6 & -6.2 & 419.076387 & 10. 0 & 20 & 23 & 3 & & 1 \\
\hline$-2,3$ & -5.5 & 419.076687 & 22.0 & 22 & 9 & 7 & 3 & \\
\hline$-2,3$ & $-5,2$ & 419.076812 & 3.5 & 12 & 22 & 6 & 4 & 3 \\
\hline-1. & $-2,3$ & 419.078030 & 21.5 & 24 & 11 & 4 & 4 & \\
\hline-0.5 & -1.2 & 419.078517 & 17.0 & 21 & 14 & 5 & 3 & 1 \\
\hline$c 0.0$ & 0.0 & 419.079004 & $12+5$ & 18 & 17 & 5 & 2. & 2. \\
\hline 0.5 & 1.2 & $419-\beta 79492$ & 8.0 & 15 & 20 & 7 & 1 & 3 \\
\hline 1,3 & 3.2 & 419.080347 & 12.0 & 20 & 19 & 3 & 3 & 2 \\
\hline 1.7 & 4.1 & 419.080710 & 26,0 & 27 & 9 & 5 & 1 & \\
\hline 1. 8 & 4.4 & 419.080834 & 7.5 & 17 & 22 & 4 & 2 & 3 \\
\hline 1.9 & 4. 6 & 419.080917 & 1.5 & 11 & 25 & 5 & 4 & 1 \\
\hline 2.2 & 5.2 & 419.091197 & 21.5 & 24 & 12 & 6 & & 1 \\
\hline 2.4 & 5.7 & 419.081405 & -3.0 & 8 & 28 & 7 & 3 & 2 \\
\hline
\end{tabular}


Keteroatom Max; 44 Ion: Poth Even and odd

Limits:

463.028640

7. 4

Mass

463.028640 a

\begin{tabular}{rrr} 
& 7.4 & \\
mDa & pPM & Calc. Mass \\
-3.0 & -6.6 & 463.025600 \\
-2.9 & -6.3 & 463.025725 \\
-2.6 & -5.5 & 463.026088 \\
-2.3 & -5.1 & 463.026295 \\
-2.1 & -4.5 & 463.026575 \\
-1.9 & -4.0 & 163.026782 \\
-0.7 & -1.6 & 463.027918 \\
-0.6 & -1.4 & 463.028001 \\
-1.5 & -1.1 & 463.028125 \\
-0.2 & -0.5 & 463.028405 \\
-0.2 & -0.3 & 463.028488 \\
\hline 0.3 & 0.7 & 463.028975 \\
1.2 & 2.6 & 463.029831 \\
1.7 & 3.6 & 463.030318 \\
2.0 & 4.4 & 463.030681 \\
2.2 & 4.7 & 463.030805 \\
3.4 & 7.3 & 463.032023
\end{tabular}

$\begin{array}{rrr}-4.2 & 0 & 0 \\ 39.9 & 200 & 400\end{array}$

DRE C H

$\begin{array}{llll}3 & 0 & 0 & 0 \\ 7 & 4 & 3 & 1\end{array}$

$\begin{array}{lll}32.5 & 31 & 3\end{array}$

$\begin{array}{lll}14.0 & 21 & 16\end{array}$

$\begin{array}{lll}3.5 & 12 & 22\end{array}$

$\begin{array}{lll}23.5 & 25 & 9\end{array}$

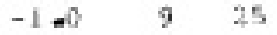

23,0 $27 \quad 11$

13.0 $21 \quad 14$

$-1.5 \quad 11$

$18.5 \quad 24$

म.

$12.0 \quad 20$

7.517

$21.5 \quad 24$

$3.0 \quad 14$

$21.0 \quad 26$

N o cl Br

$4 \quad 2$

$\begin{array}{llll}3 & 3 & 3 & \\ 5 & 1 & 1 & \\ 6 & 4 & 2 & 1 \\ 6 & & 2 & \\ 7 & 3 & 3 & 1 \\ 3 & 1 & 2 & 1 \\ 5 & 3 & & 1 \\ 4 & 4 & 3 & 1 \\ 4 & & 3 & 1 \\ 6 & 2 & 1 & 1 \\ 7 & 1 & 2 & 1 \\ 3 & 3 & 1 & 1 \\ 4 & 2 & 2 & 1 \\ 6 & & & 1 \\ 5 & 1 & 3 & 1 \\ 3 & 1 & & 1\end{array}$




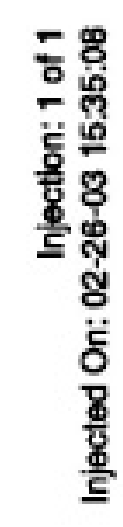

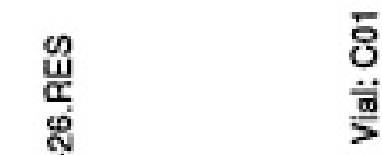

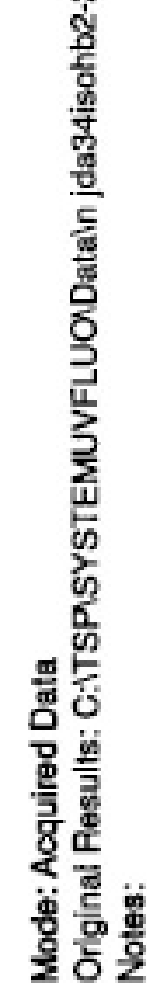

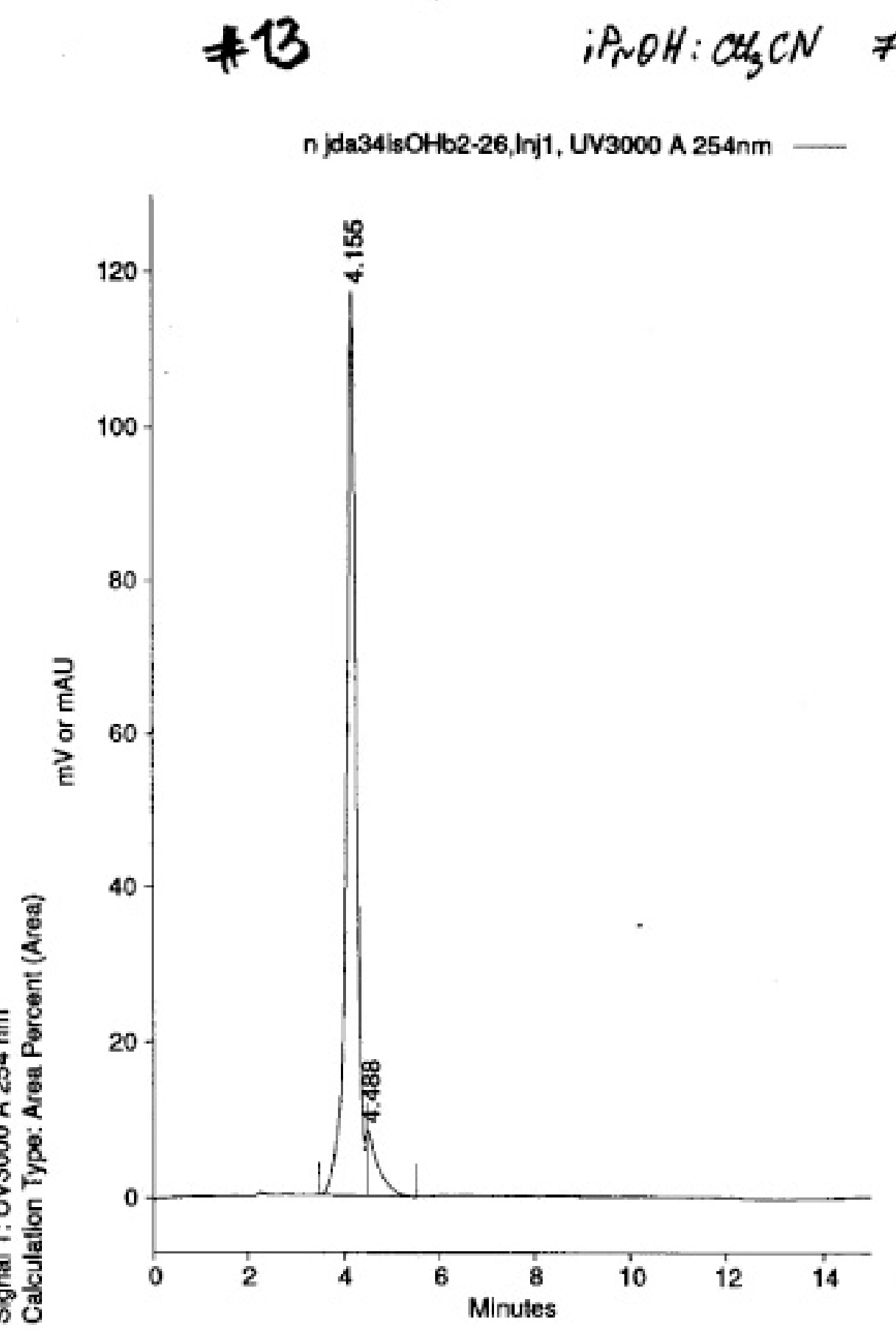

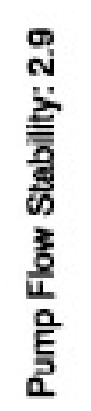

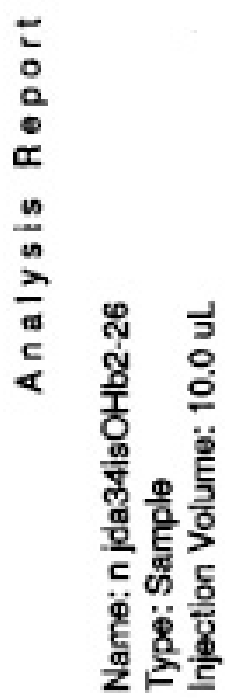

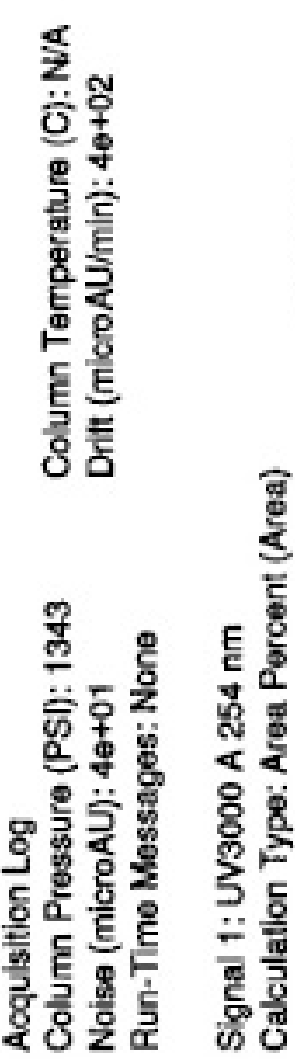



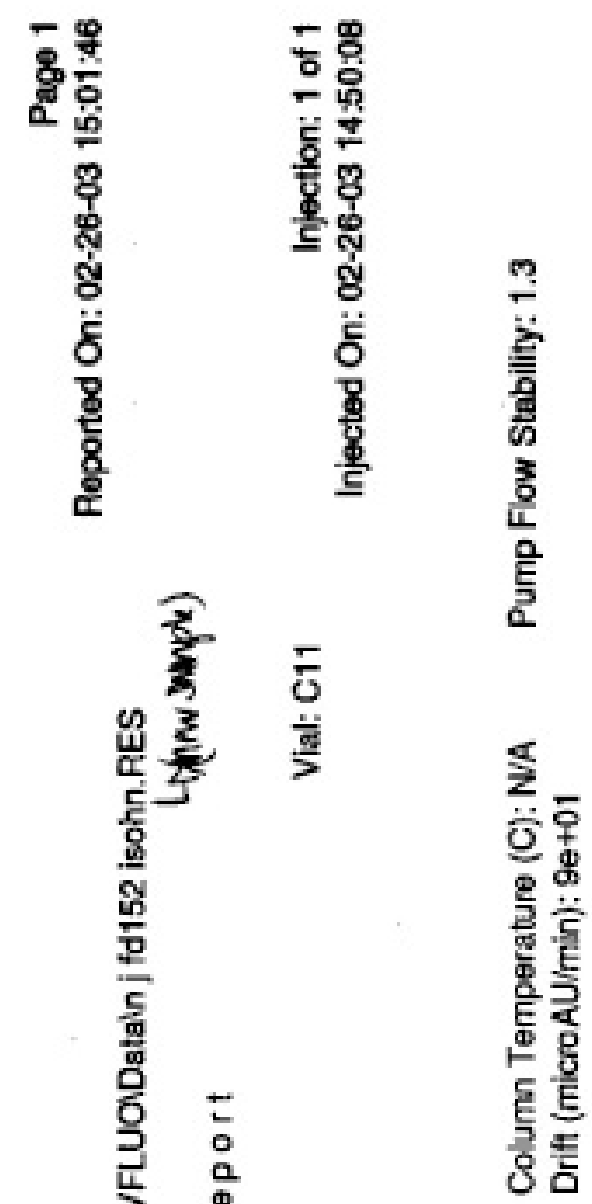

券

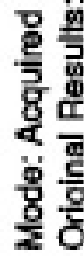

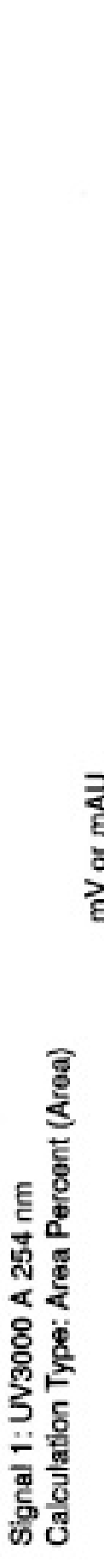

$\$ 14$

$\mathrm{PPOH}_{\mathrm{O}} \mathrm{CH} C \mathrm{CN} \quad 70: 30$

ำสำ

n j fd152 isohn,Inj1, UV3000 A 254nm

政

$5 \%$

잉

क्षे

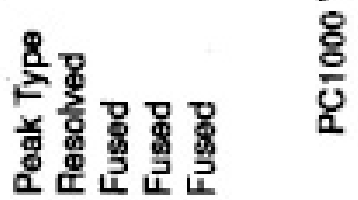

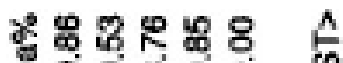

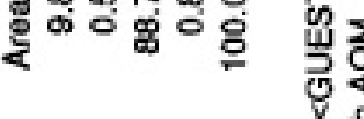

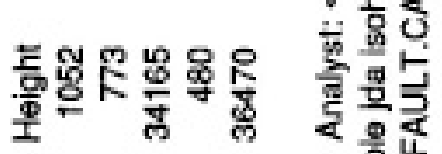

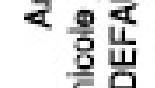

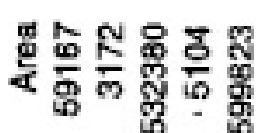

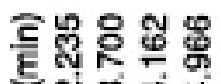
的的

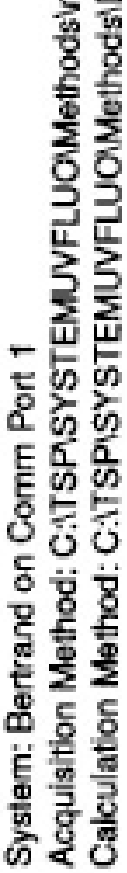



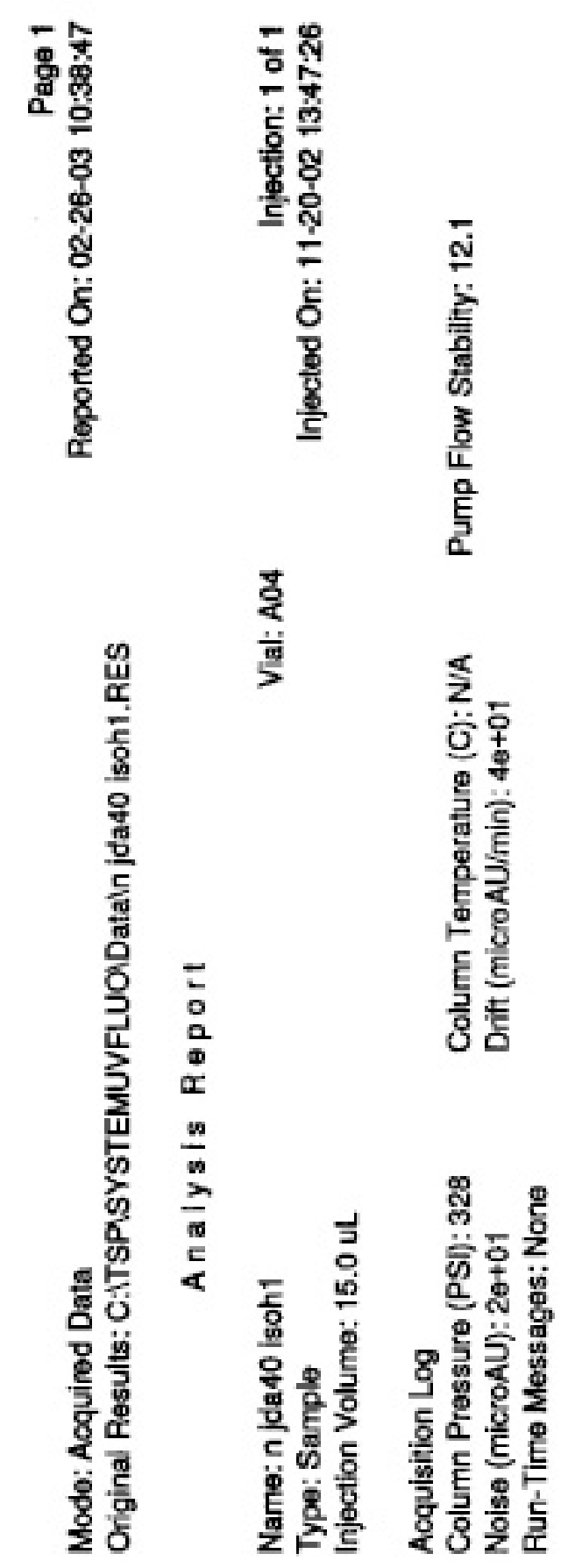
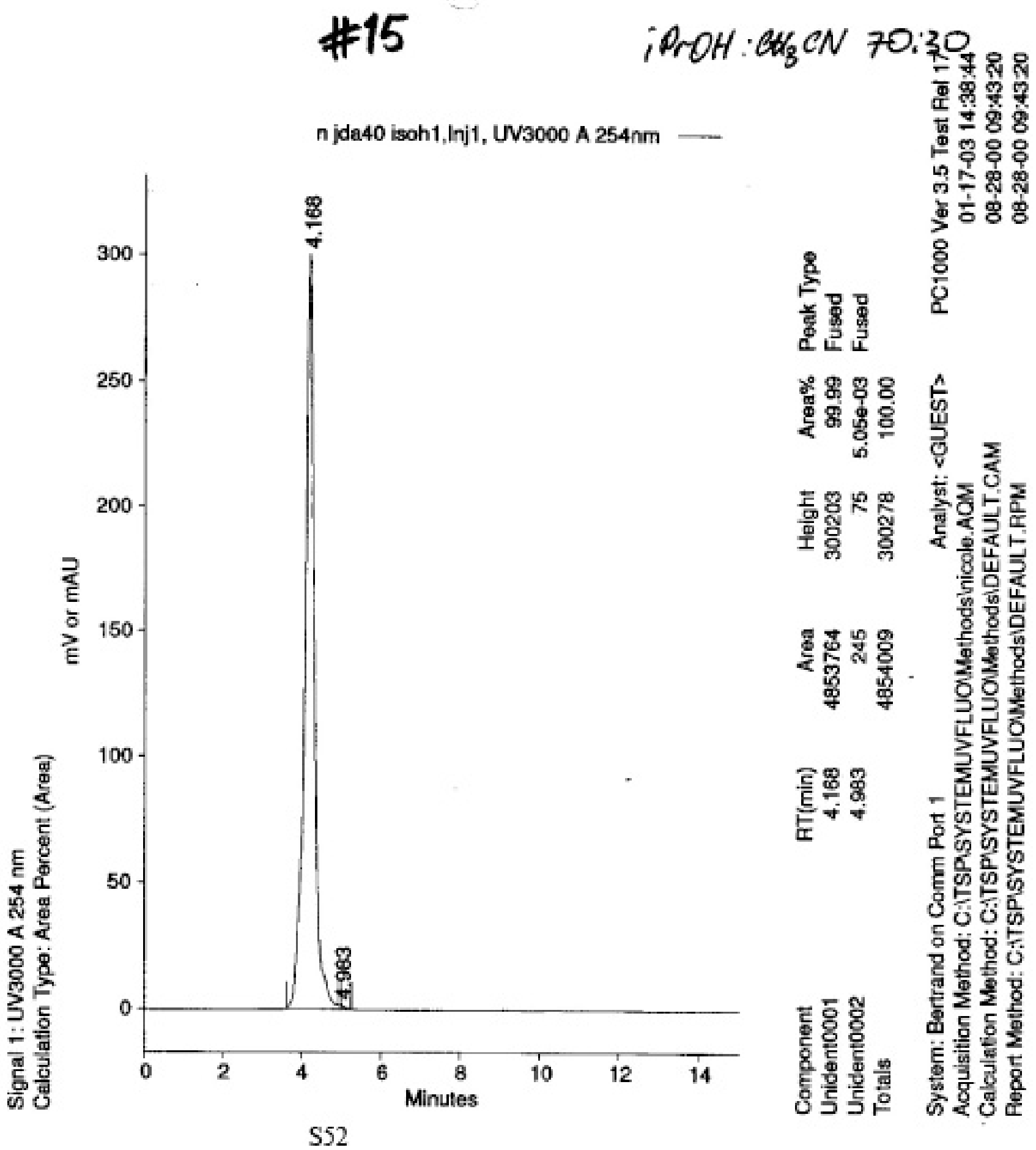

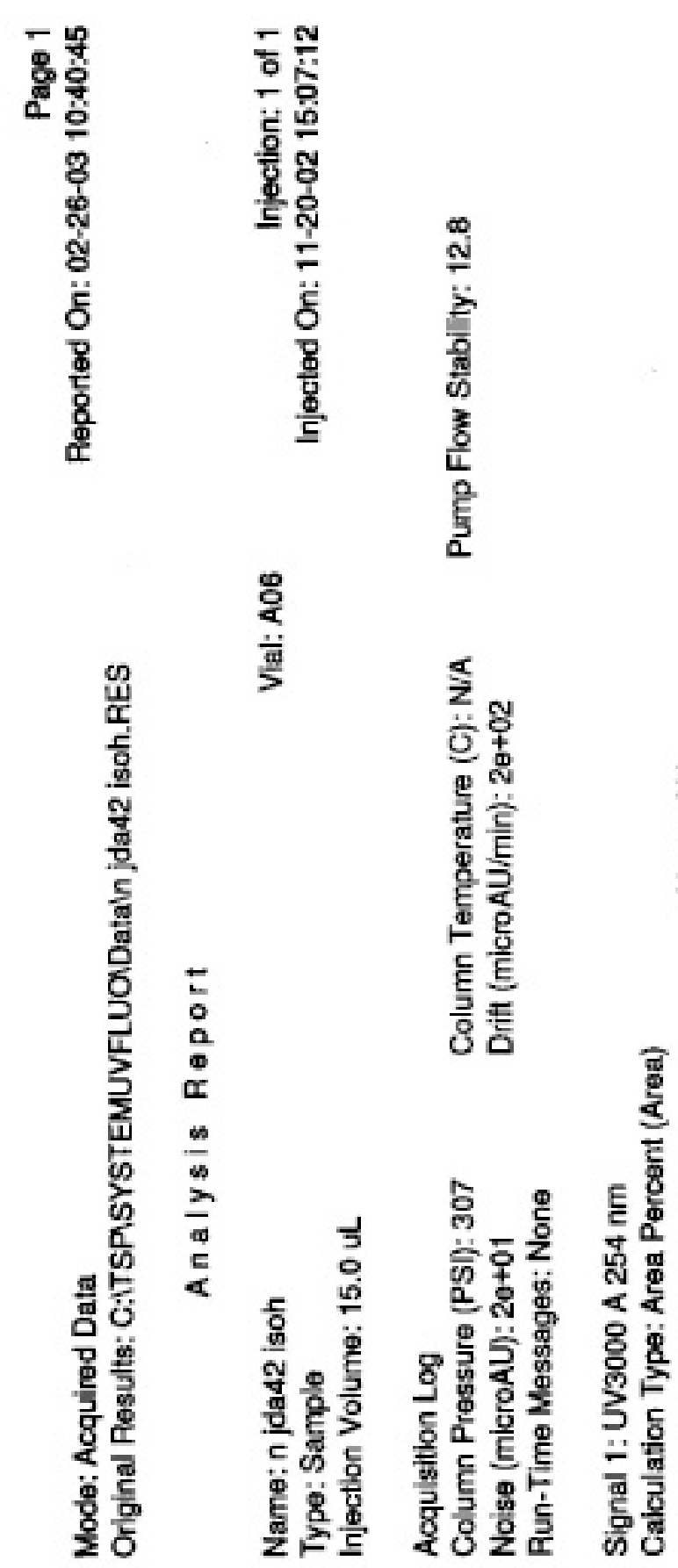

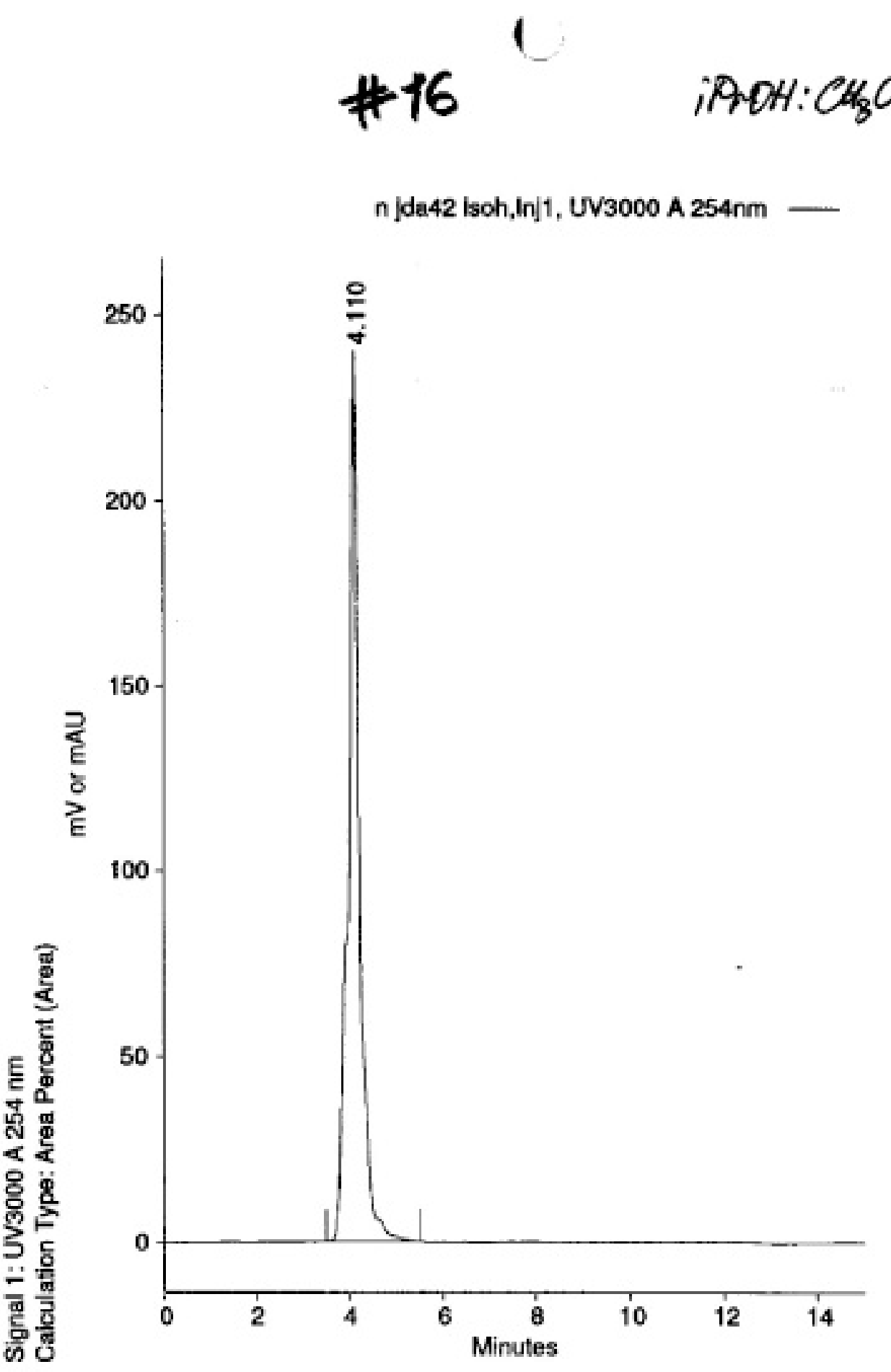

S53
뿡ำ

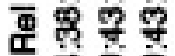

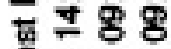

땡ㅇㅇㅇ

政

它

害皆

ॠू.8

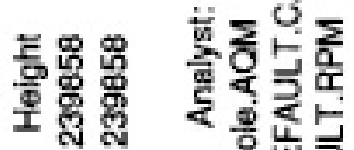

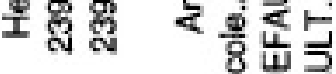

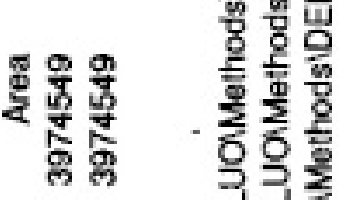

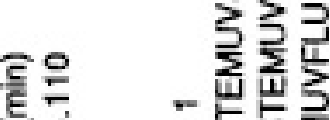

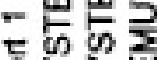

둘

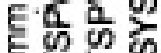

85

당

80

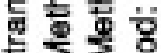

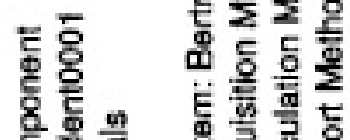

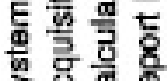




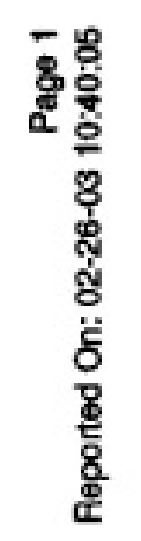

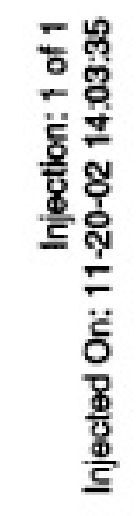

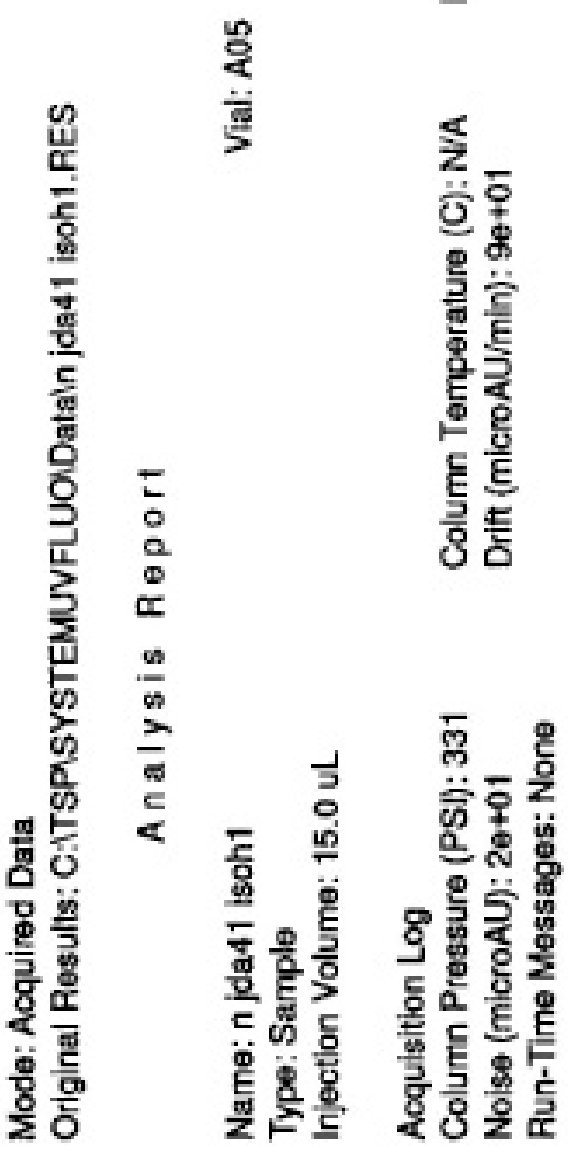

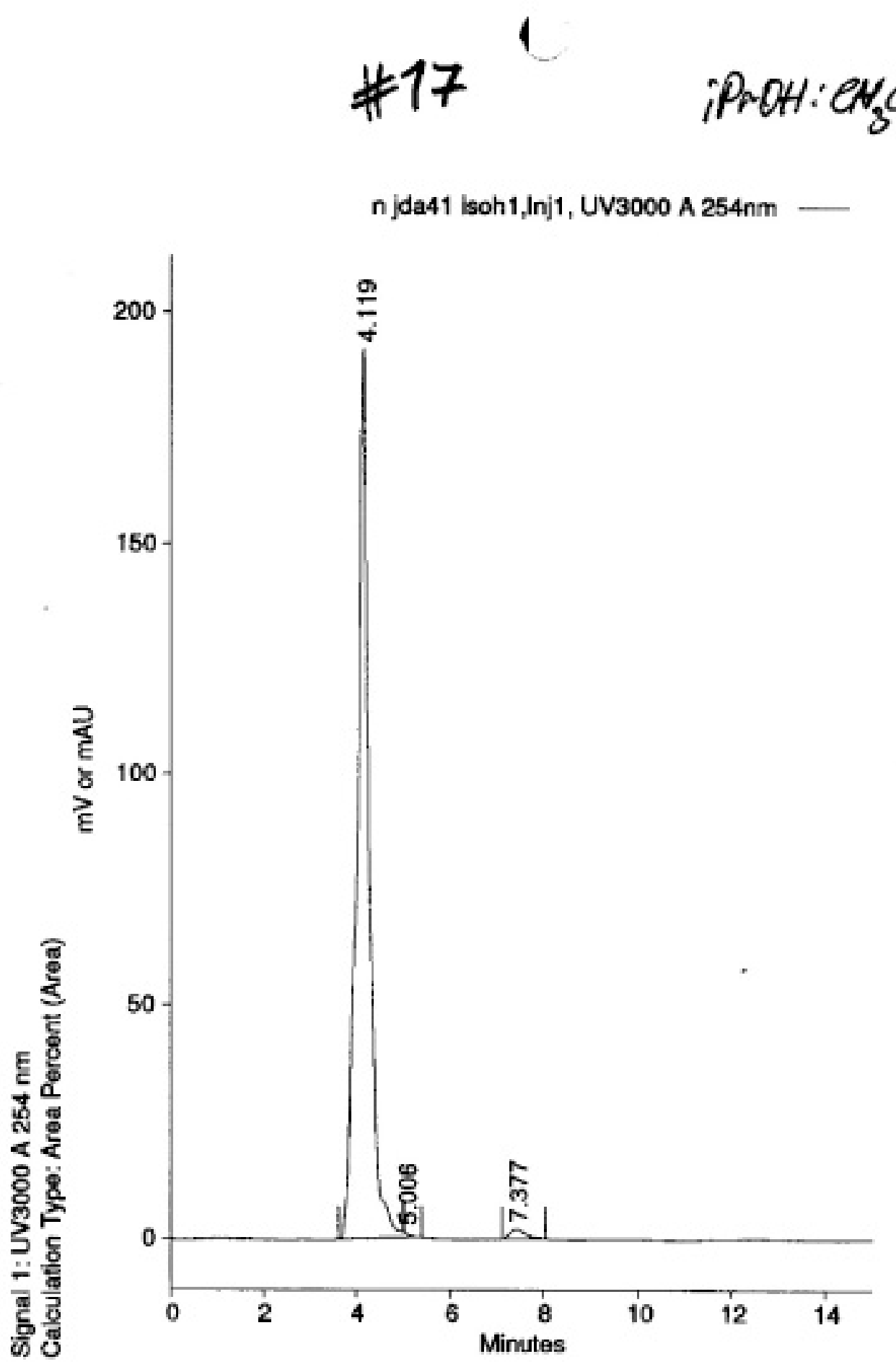

S54
ㄴํํํำ बㅇํㅇ 둥ㅇㅇ 윙우 숭 \$ㄷㅇㅇㅇㅇ

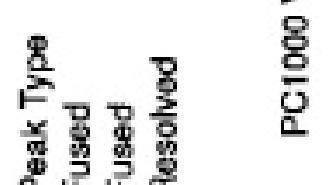
as

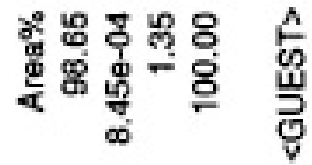

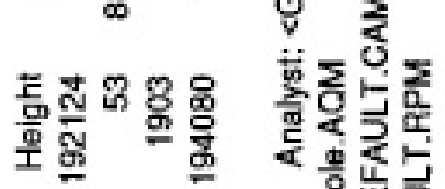
.

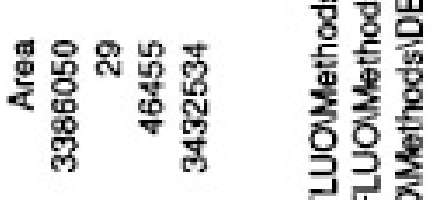

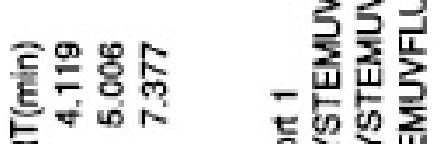

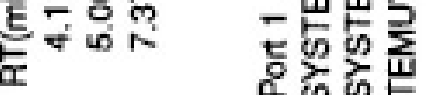
造就

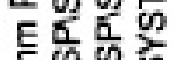
은 ठठिक 당

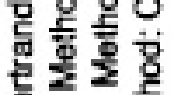

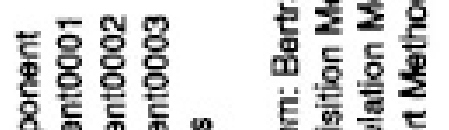

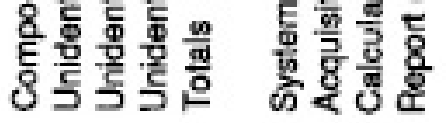



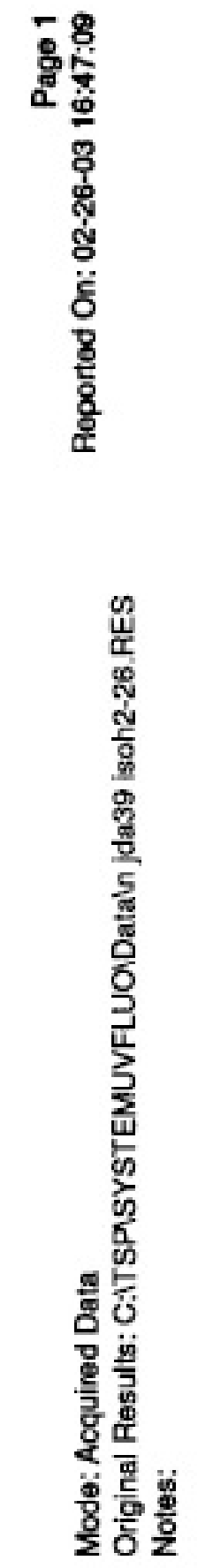

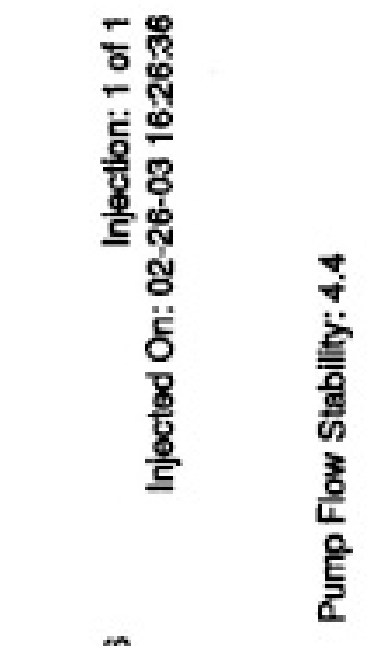

$\frac{\circ}{\frac{0}{5}}$

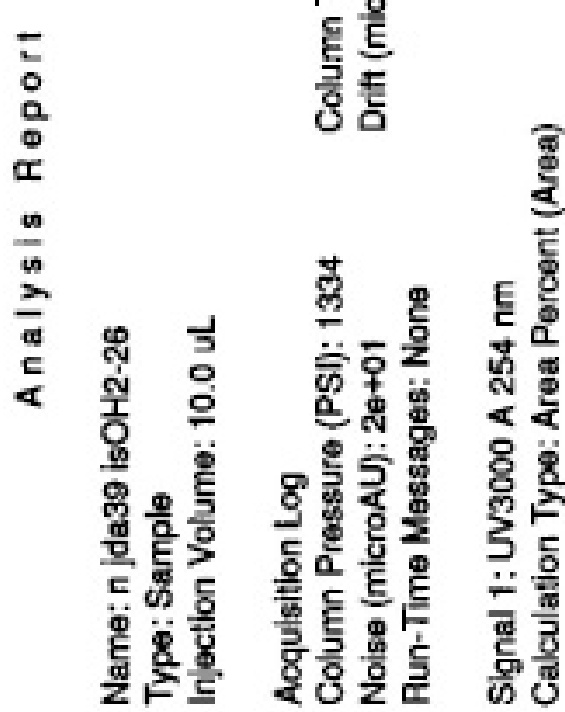

\#18

, PrOH: CHG CN 70:30

n jda39 isOH2-26, Inj1, UV3000 A 254nm -

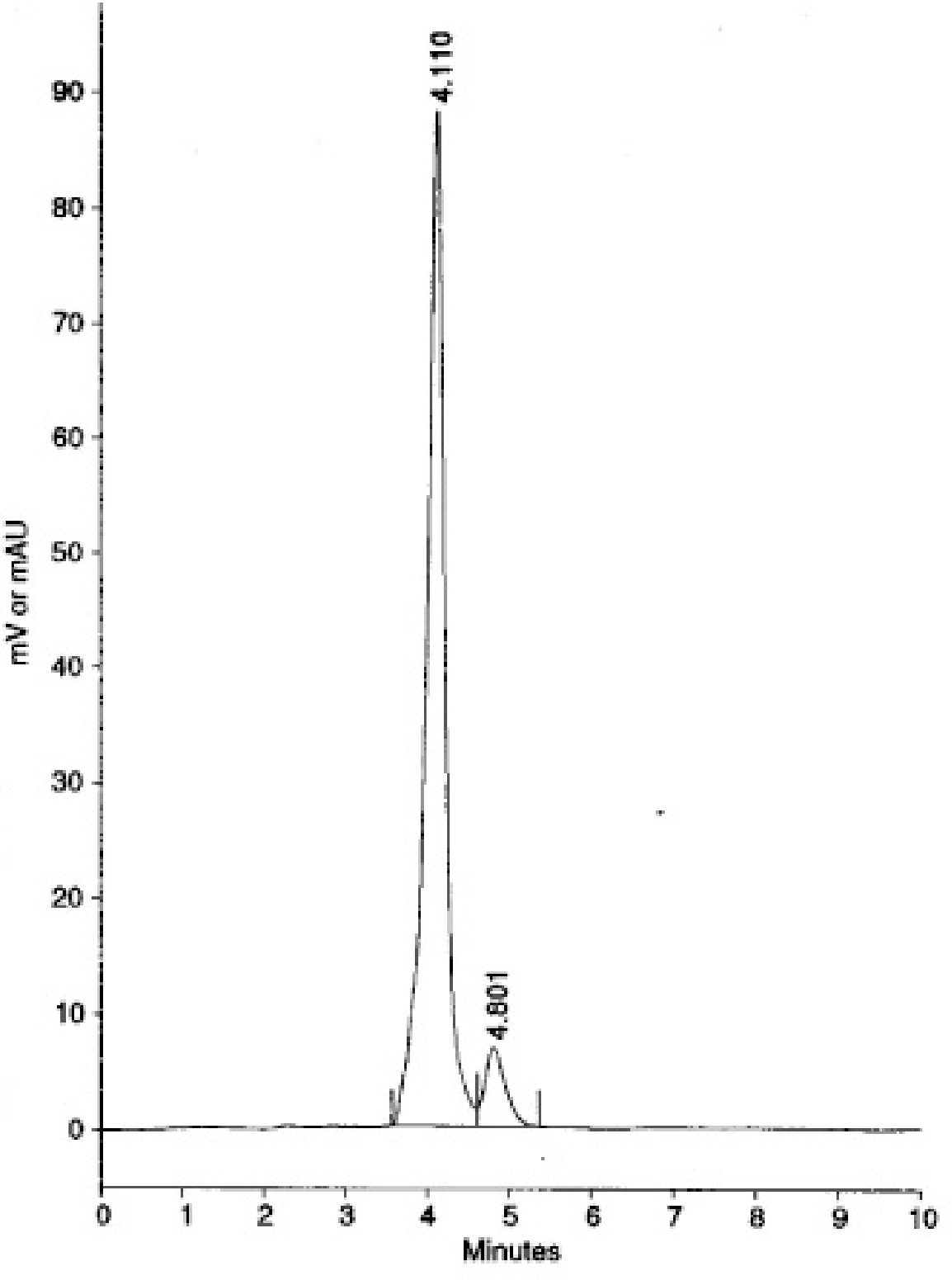

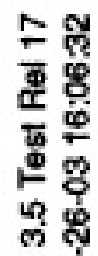

के 영

8 임

1

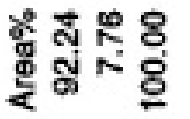

点

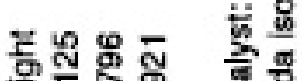

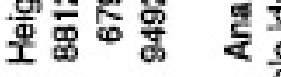

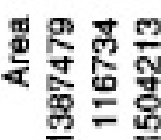

듵을혛

동 总

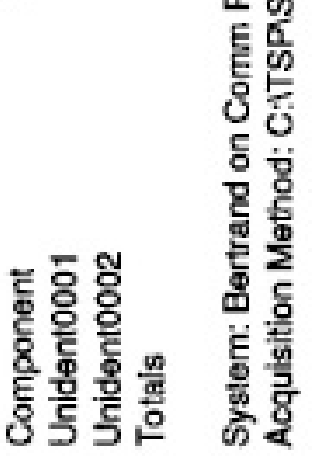




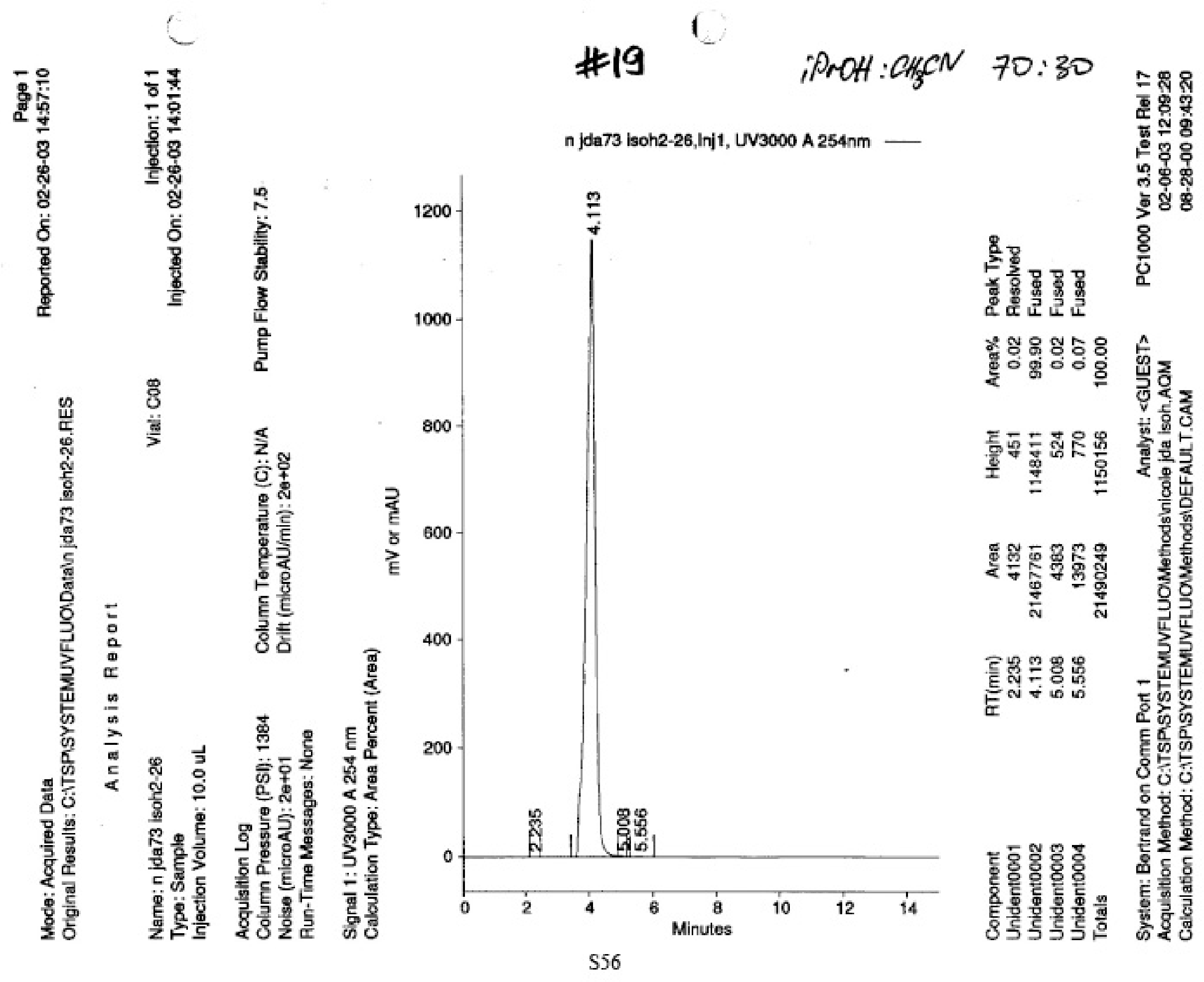



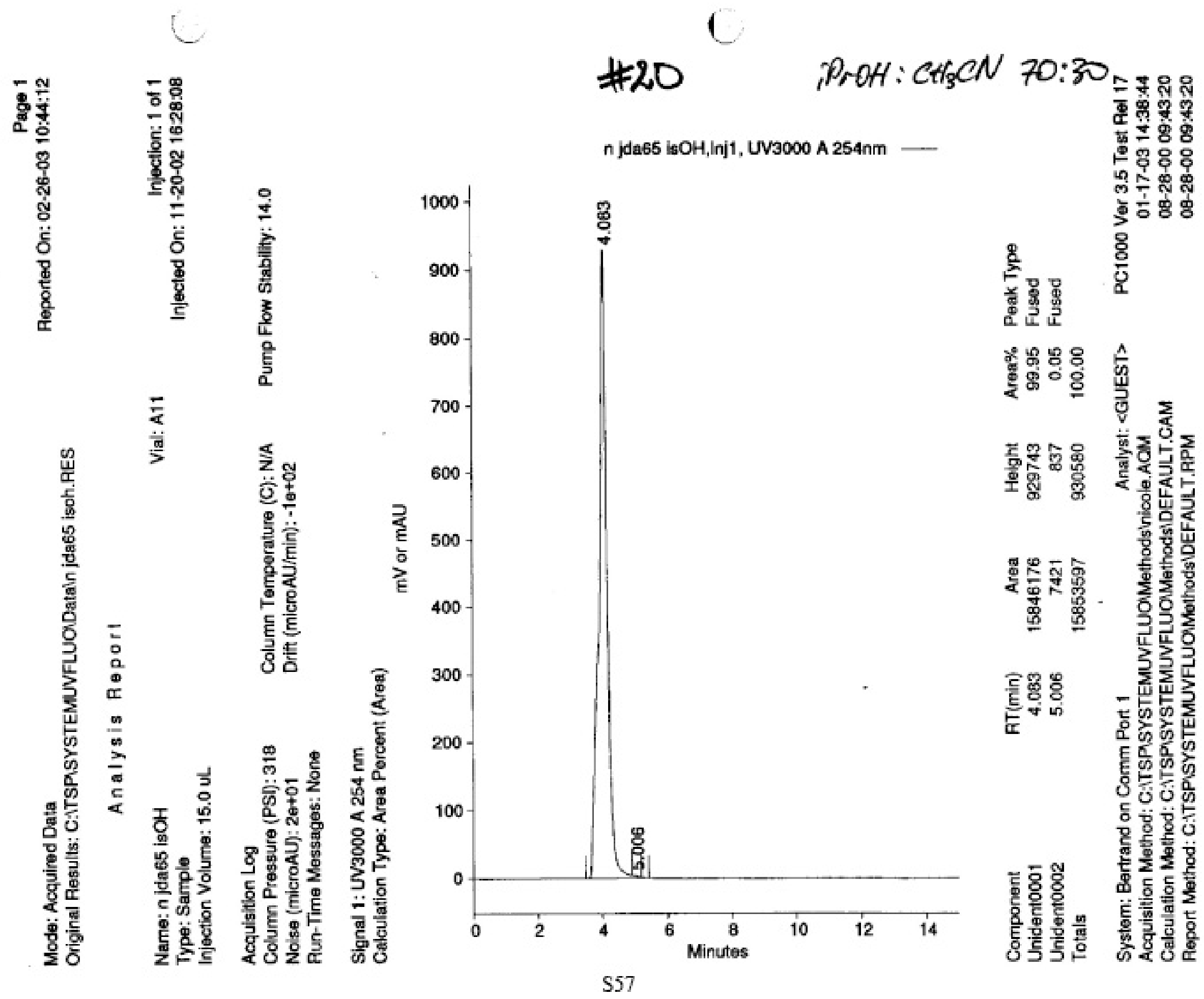


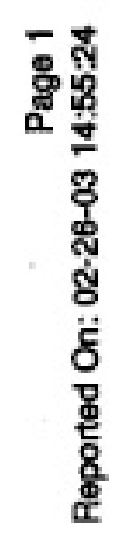

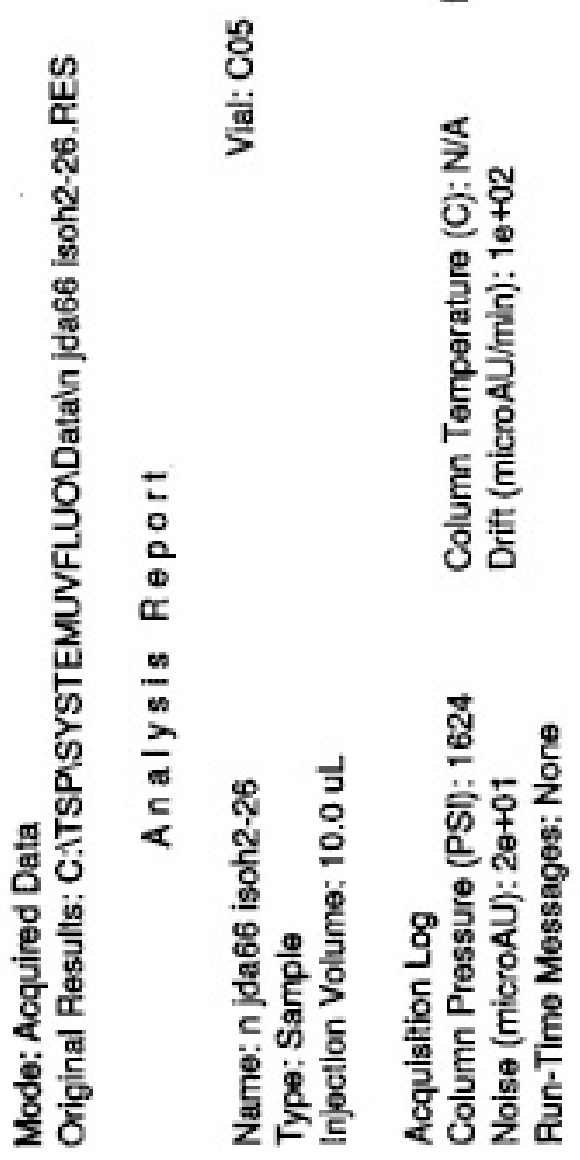

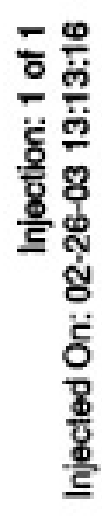

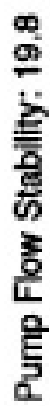

年

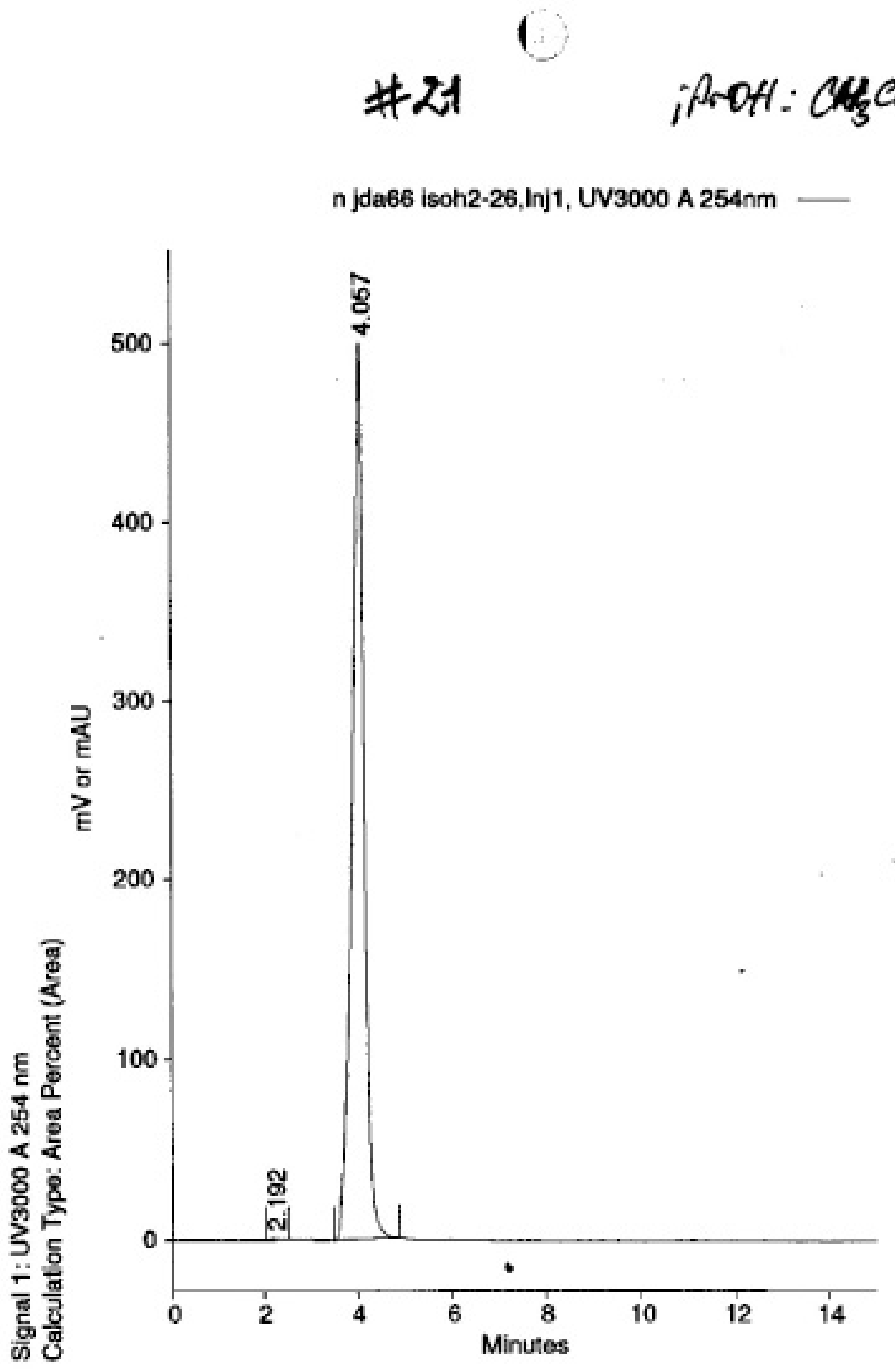

S58
สิก 车 8 응 8 땅응 잉

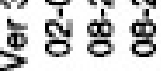

용효 睘平近 怤=

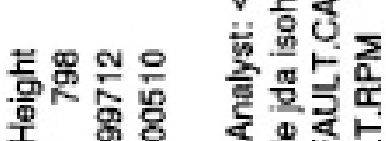

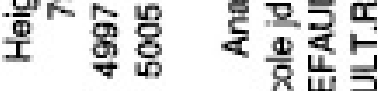

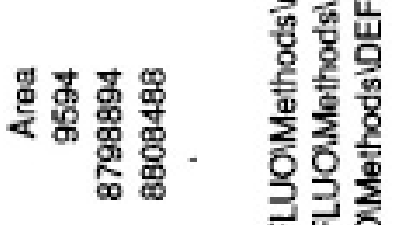
즐

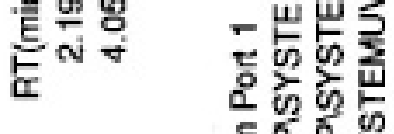

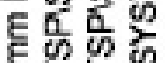
8 ㄴํㅇ 울 뉸 등융

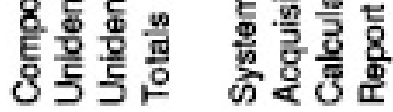




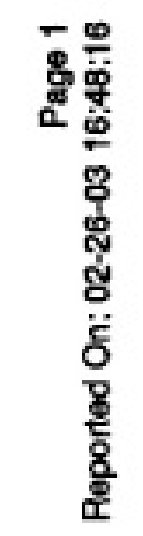

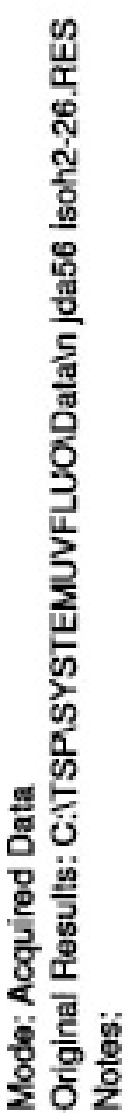

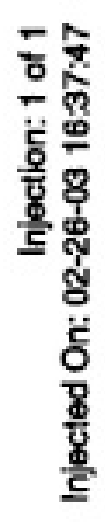

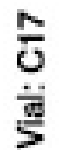

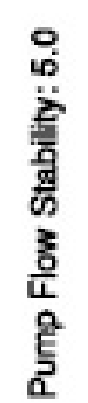
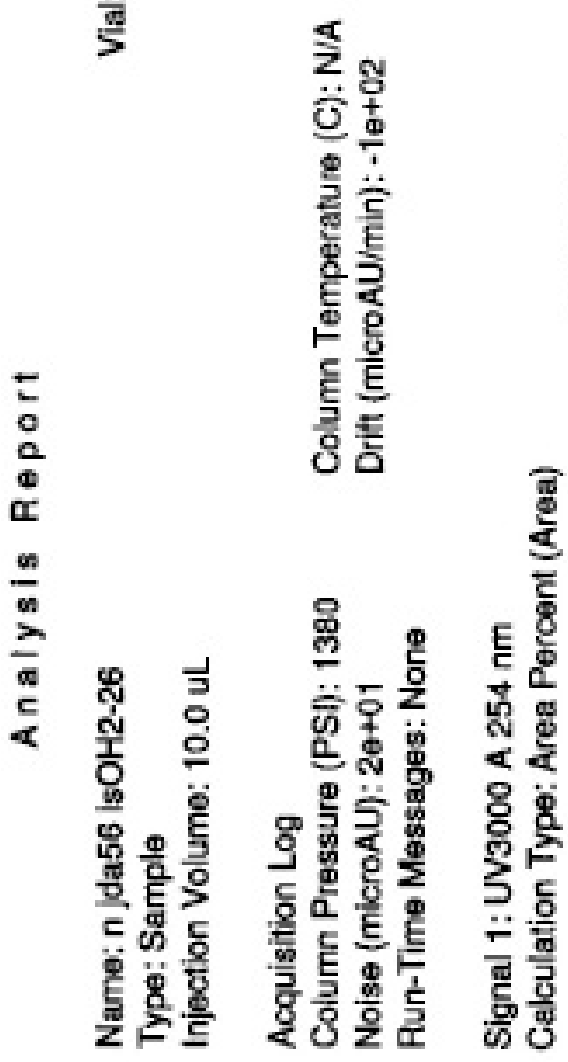

\#22 iPNOH: $\mathrm{CH}_{3} \mathrm{CN}$ 70:30

n jda56 is $\mathrm{OH} 2-26, \ln \mid 1$, UV3000 A 254nm
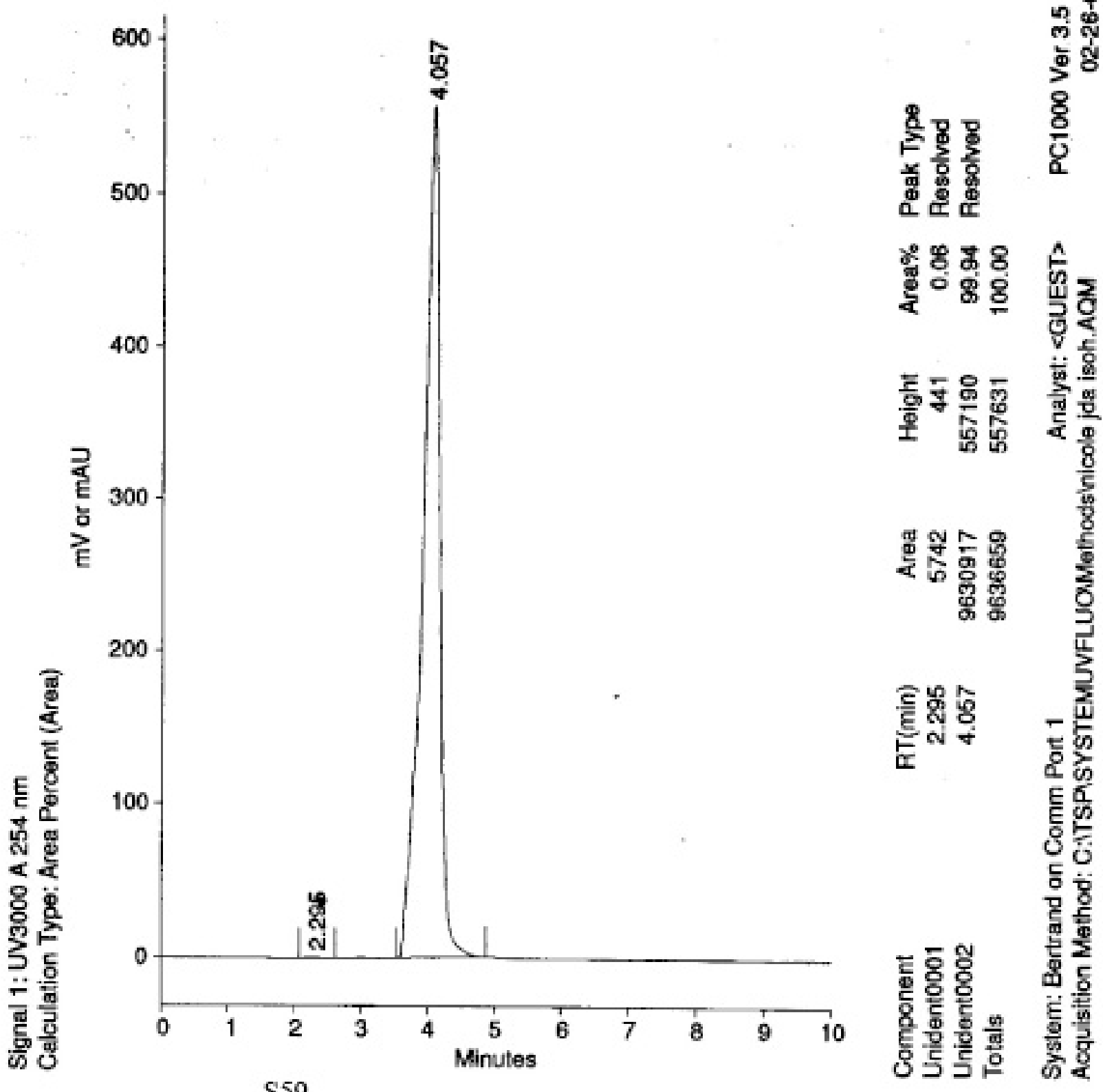

\% \&ु용 预

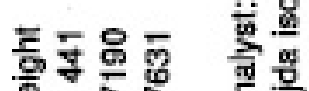
生点

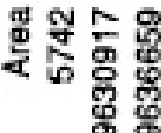

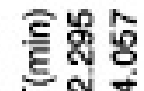

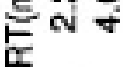

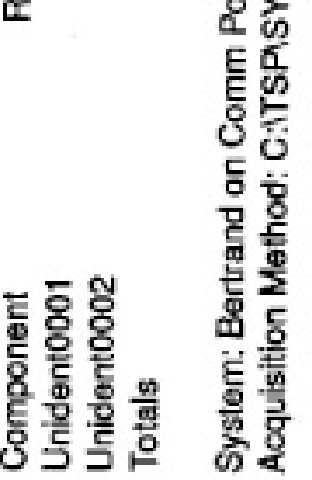




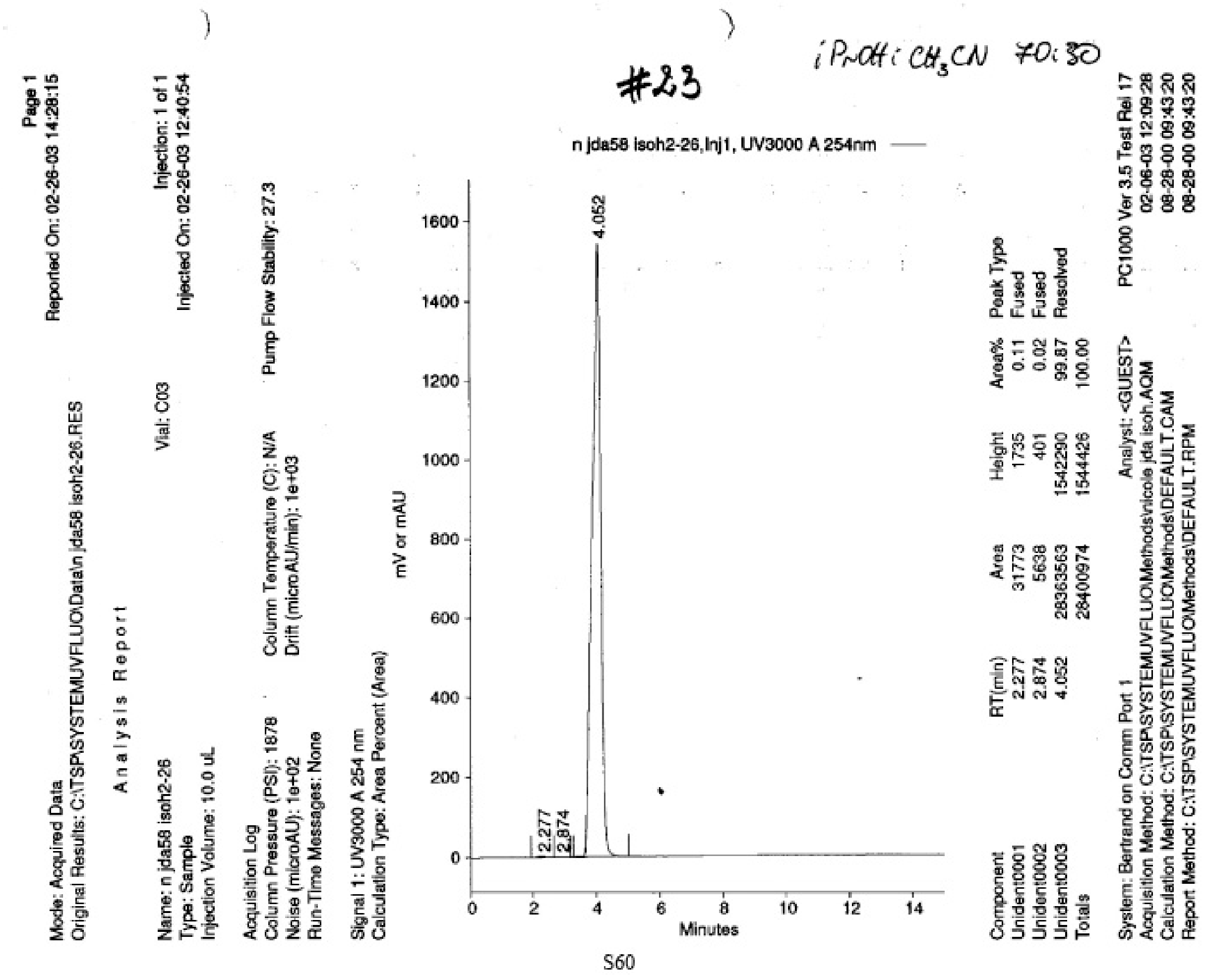



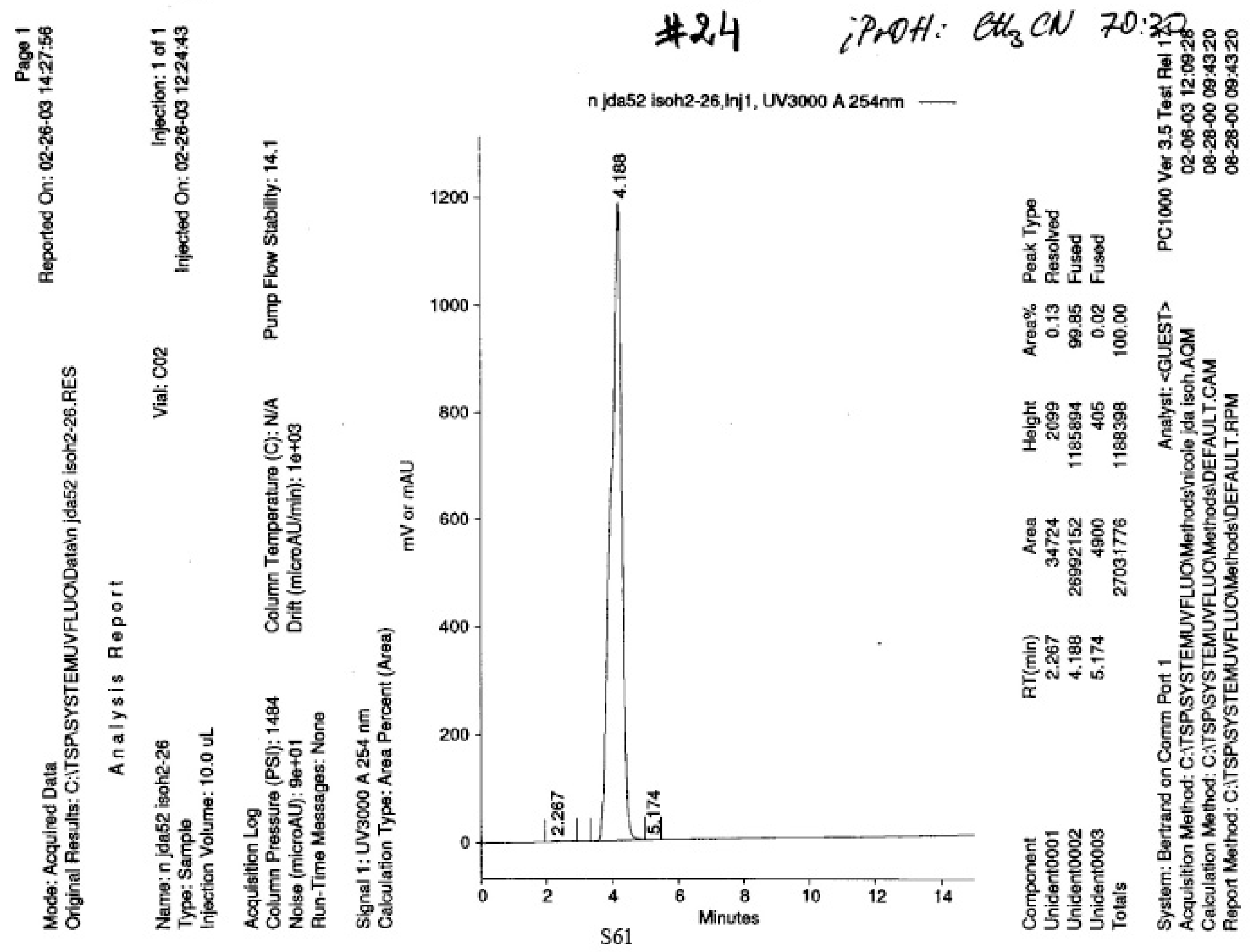


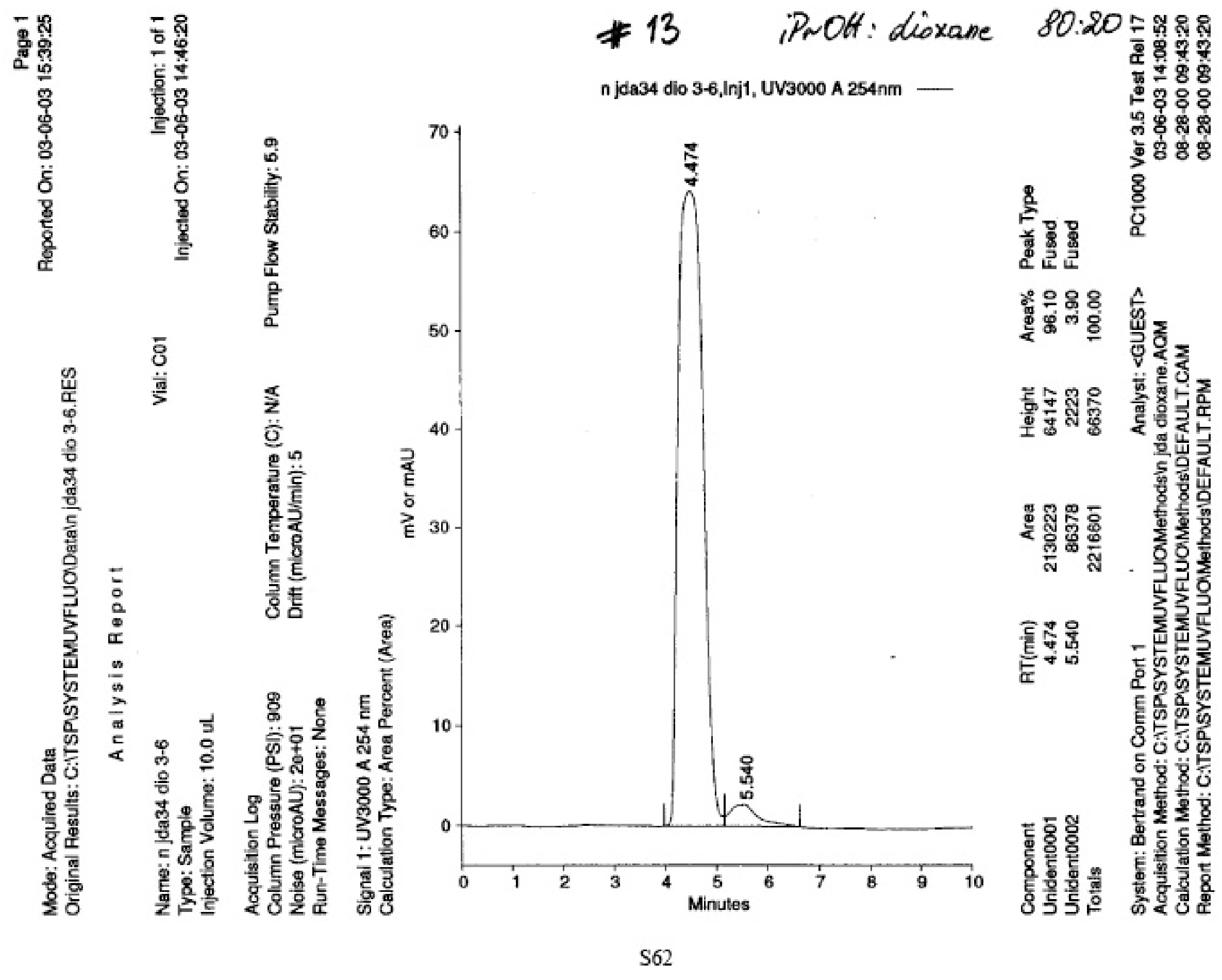




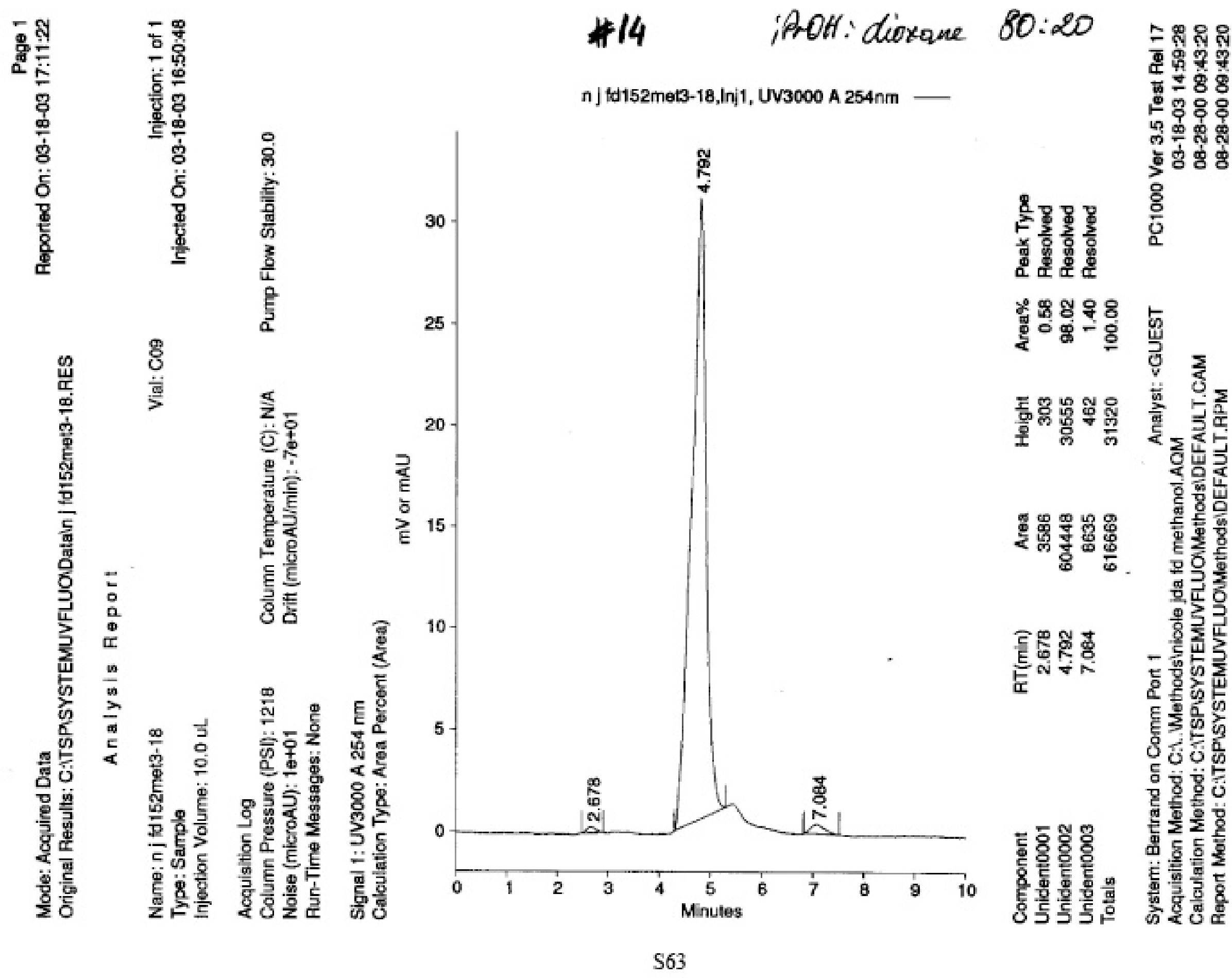



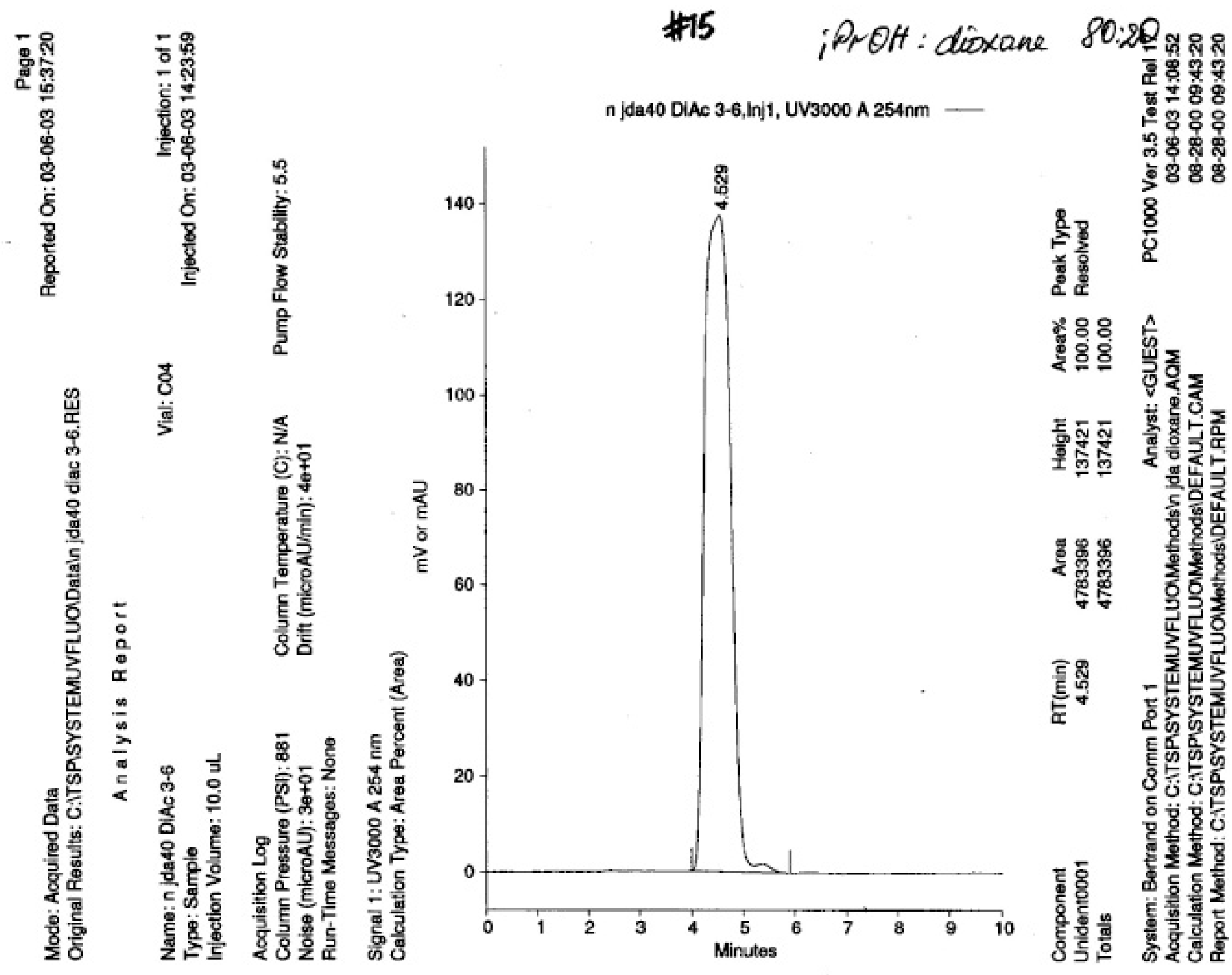

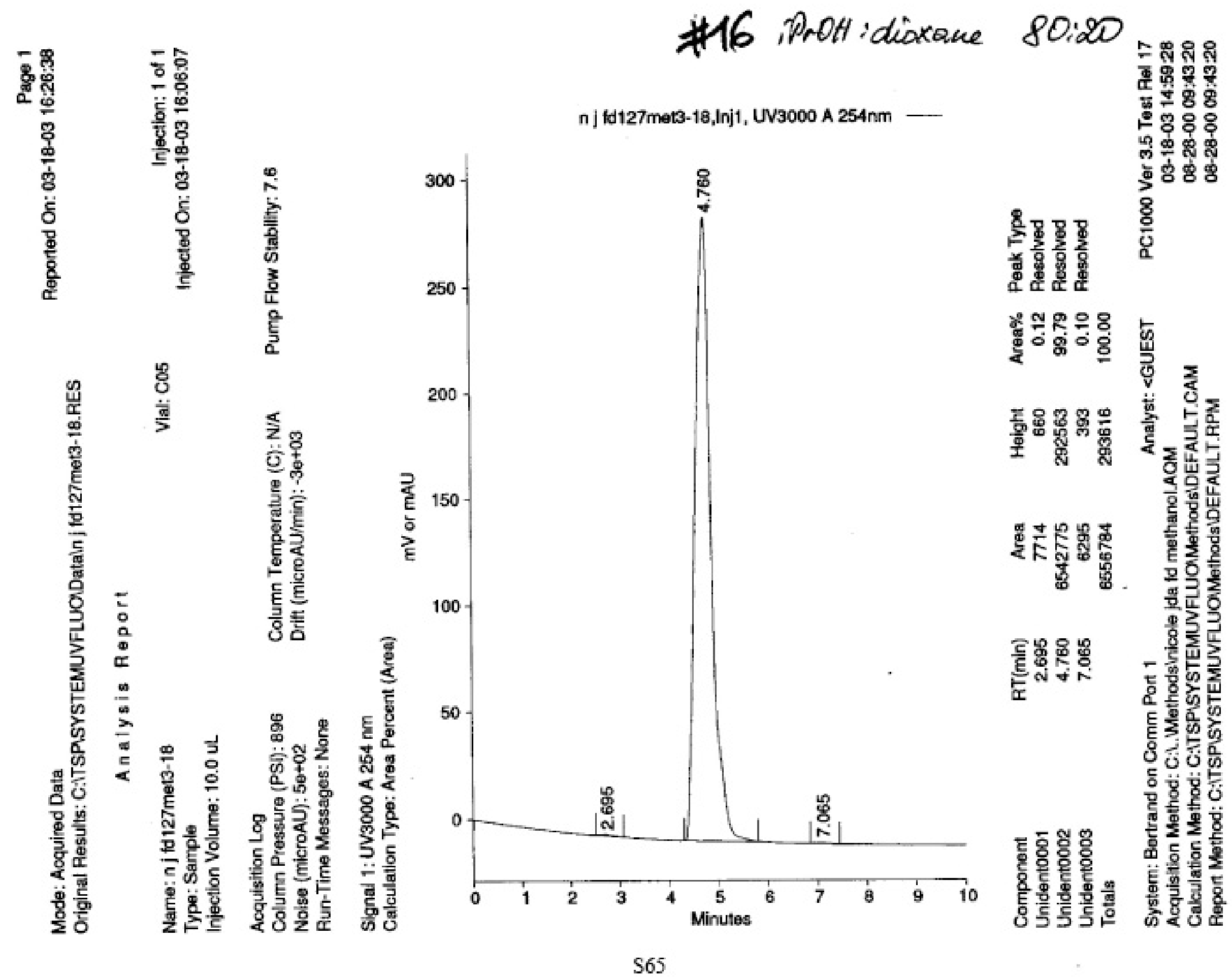


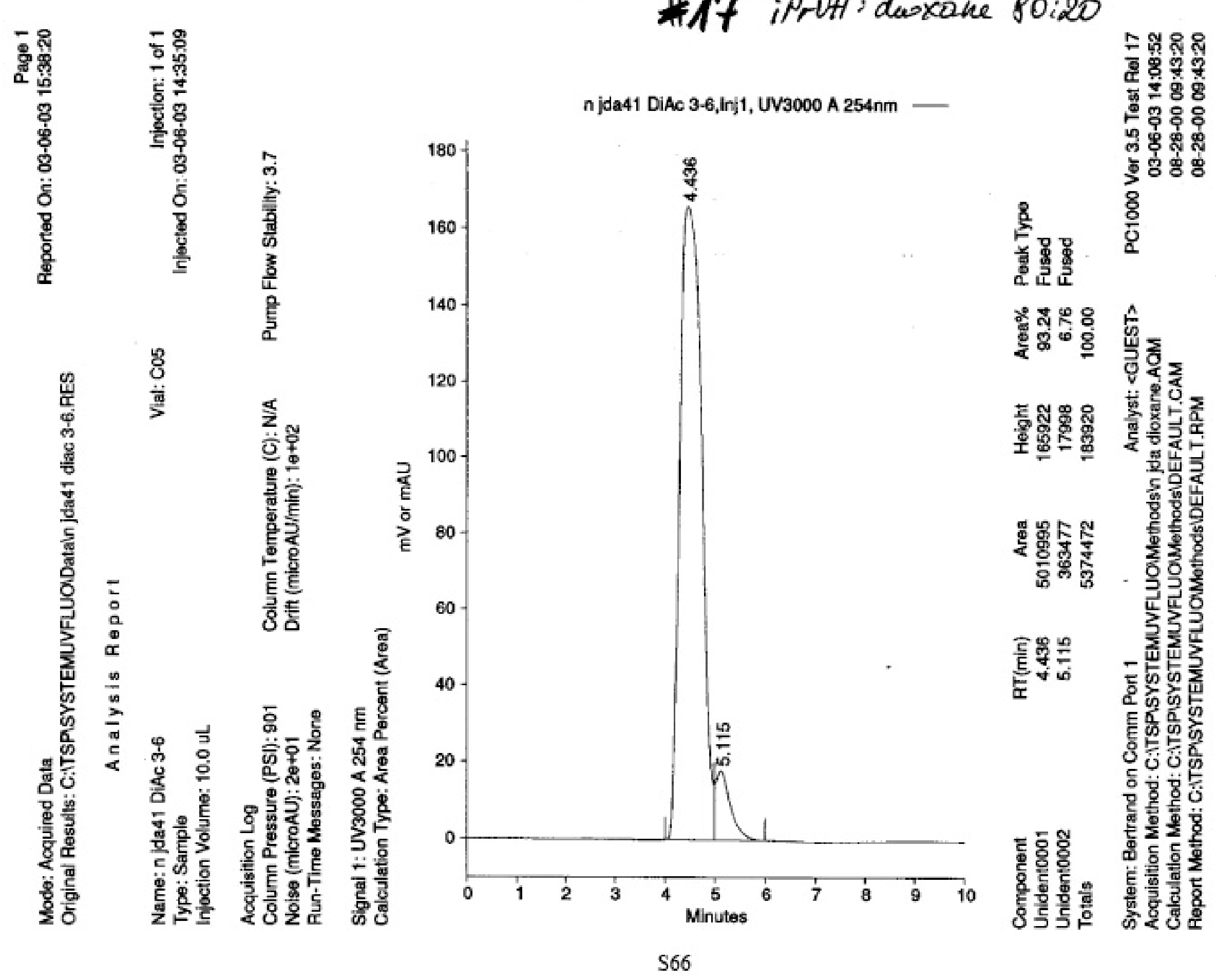


\#18 PrOH: dioxone 80:20

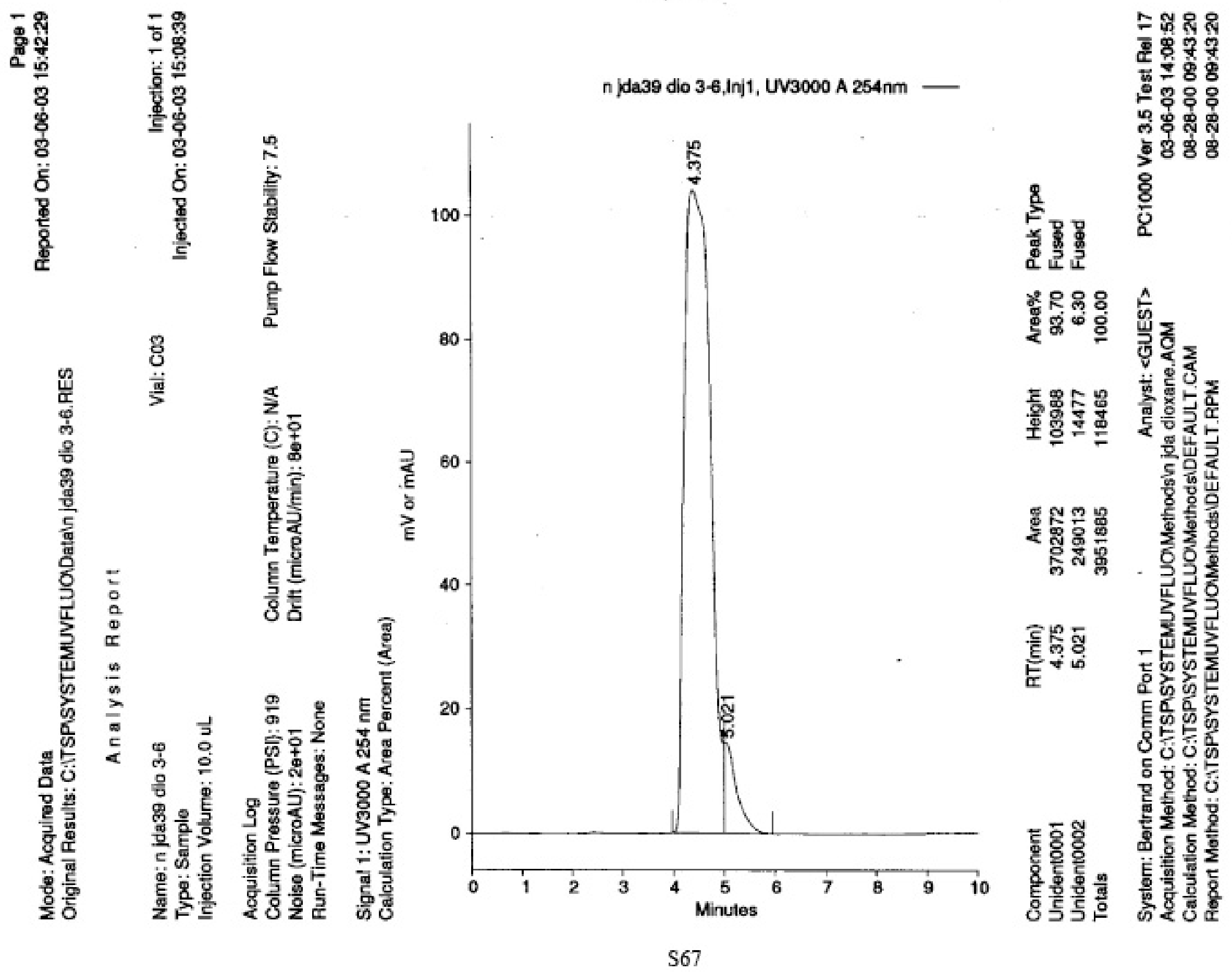



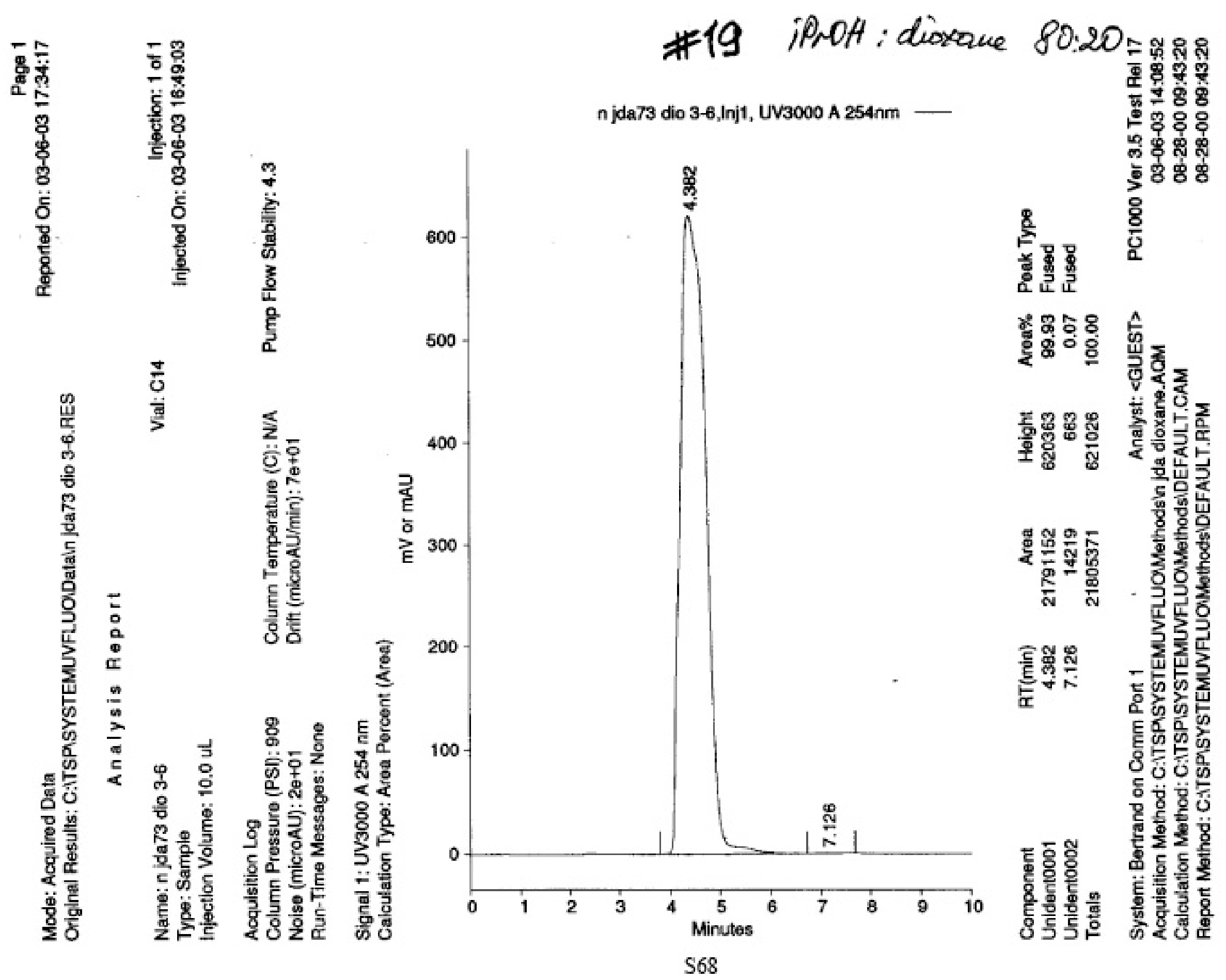

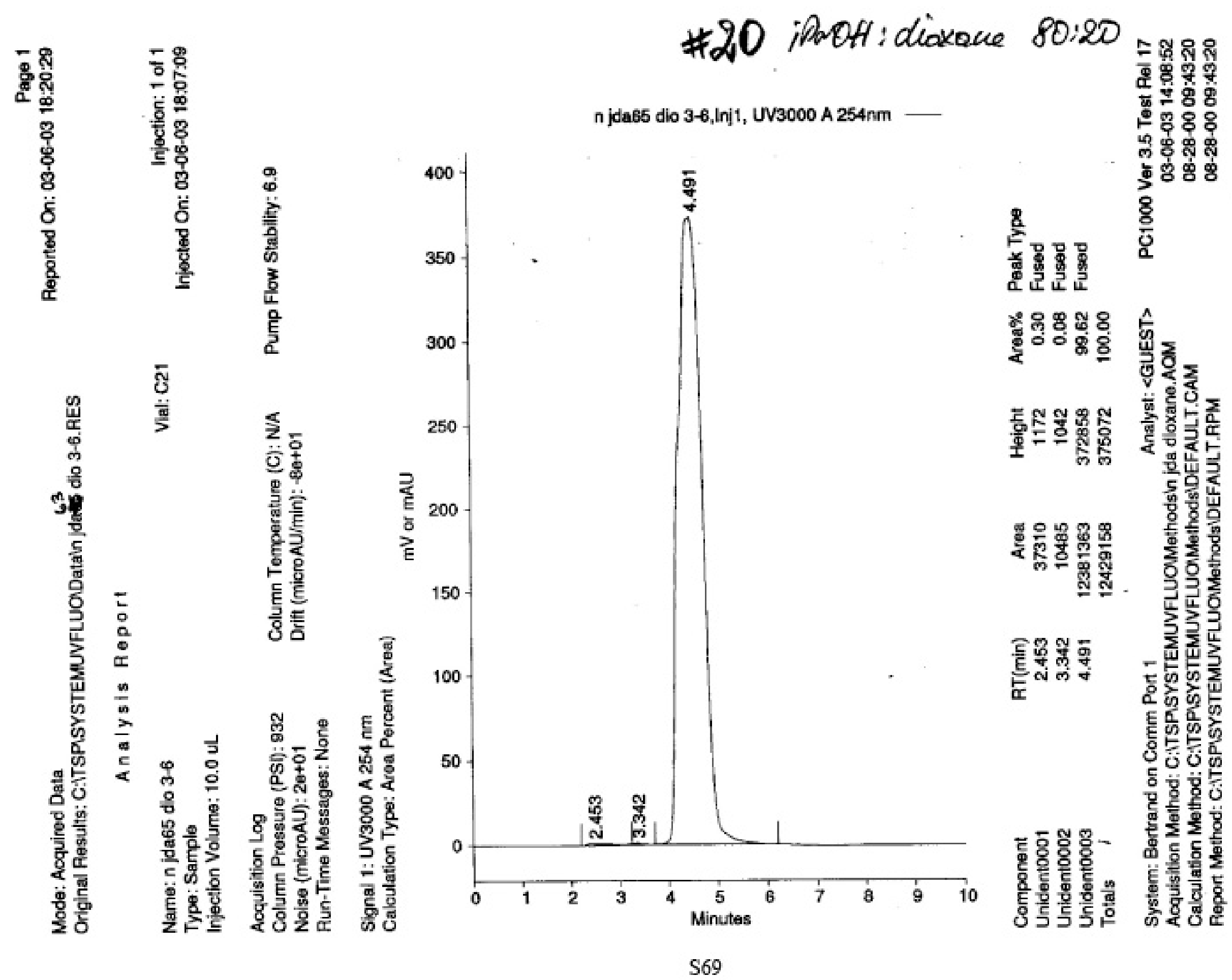

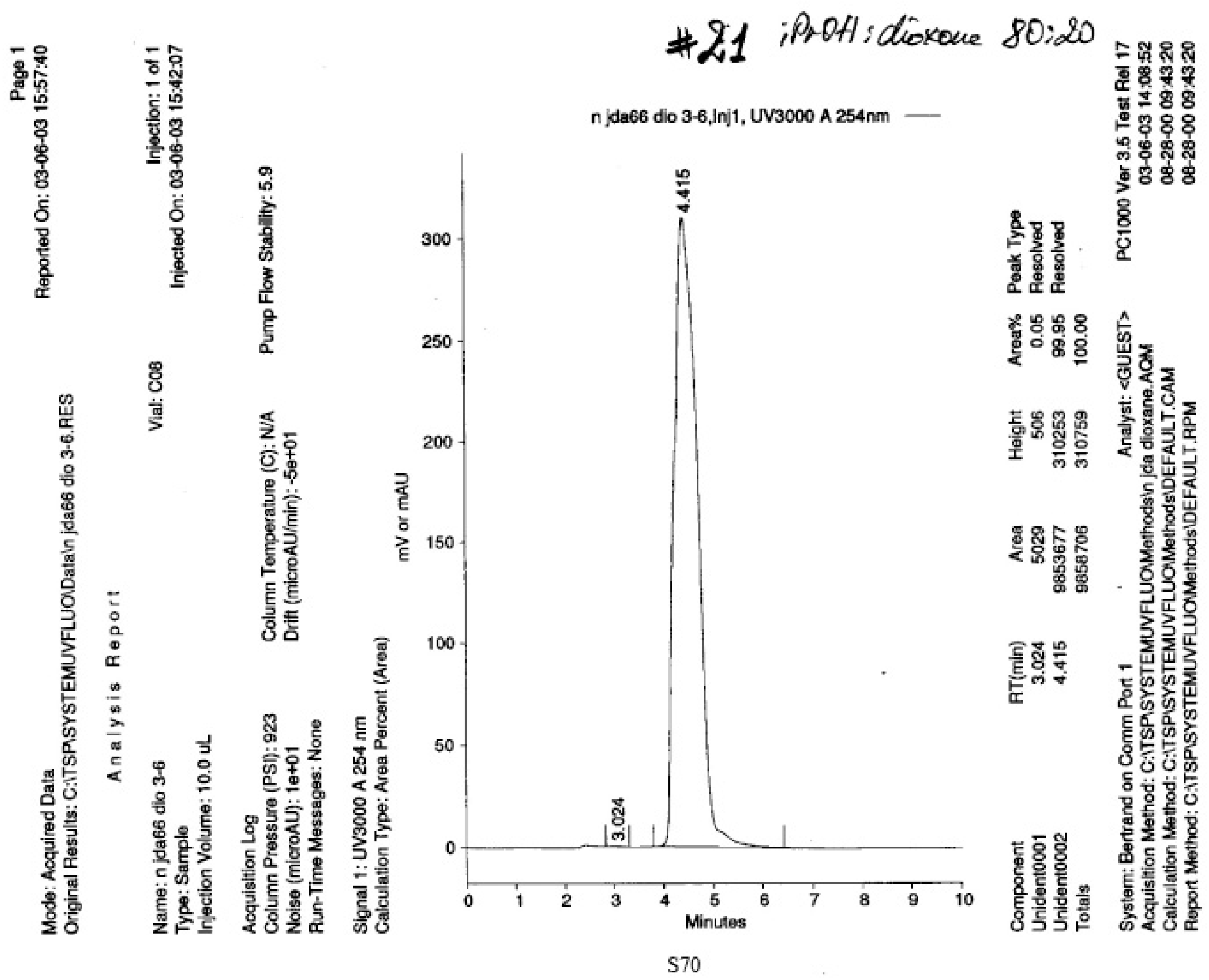
\#22 inoH: divace 80:20

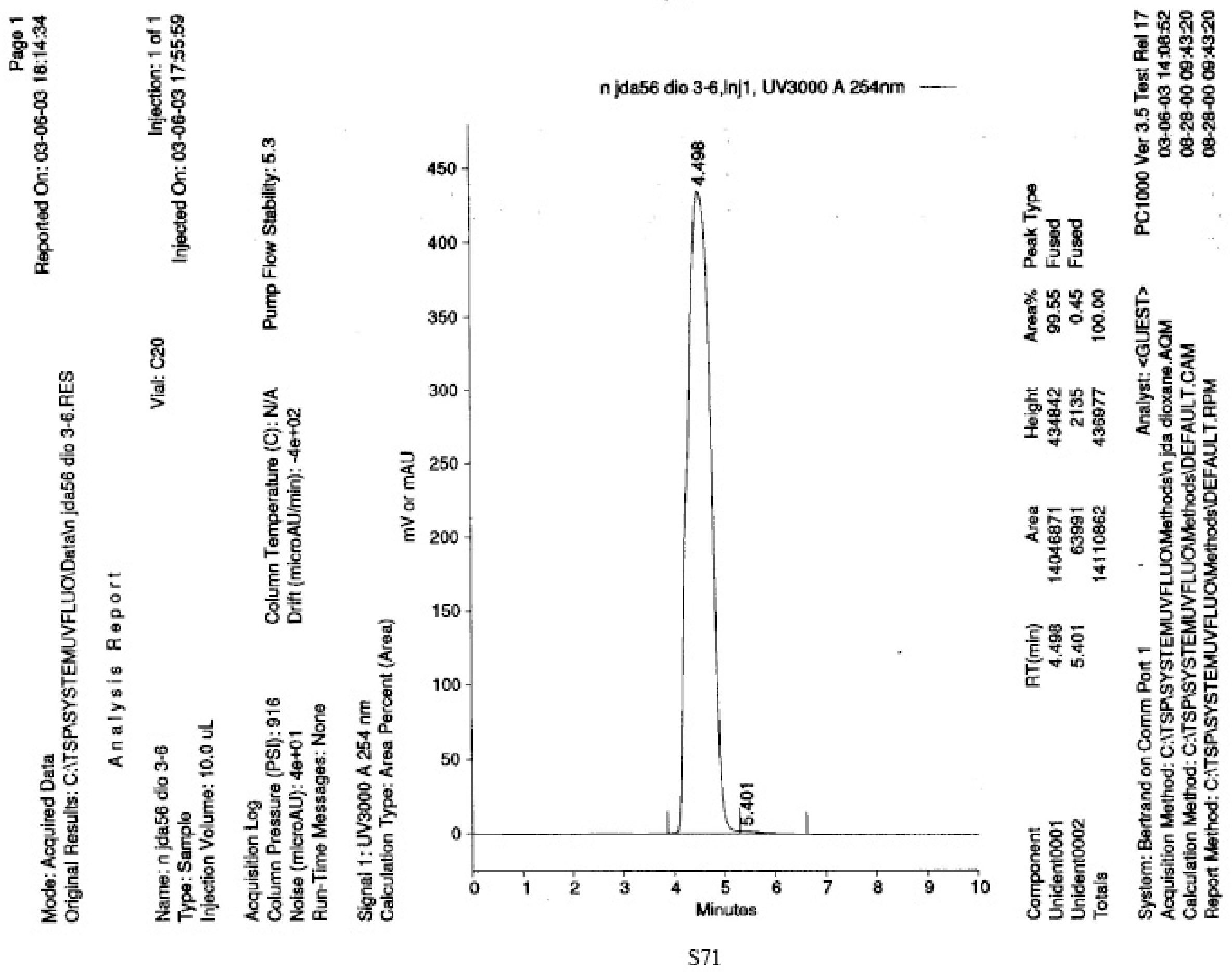


\#23

iPrOAf: dion 0 ne 80:20

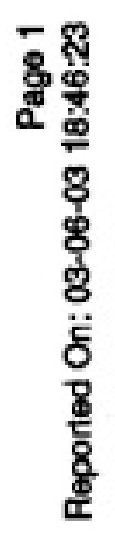

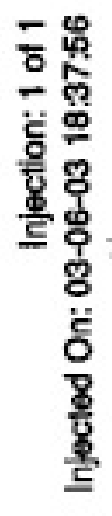

n jda58 dio $3-6$, , Inj1, UV3000 A 254nm -

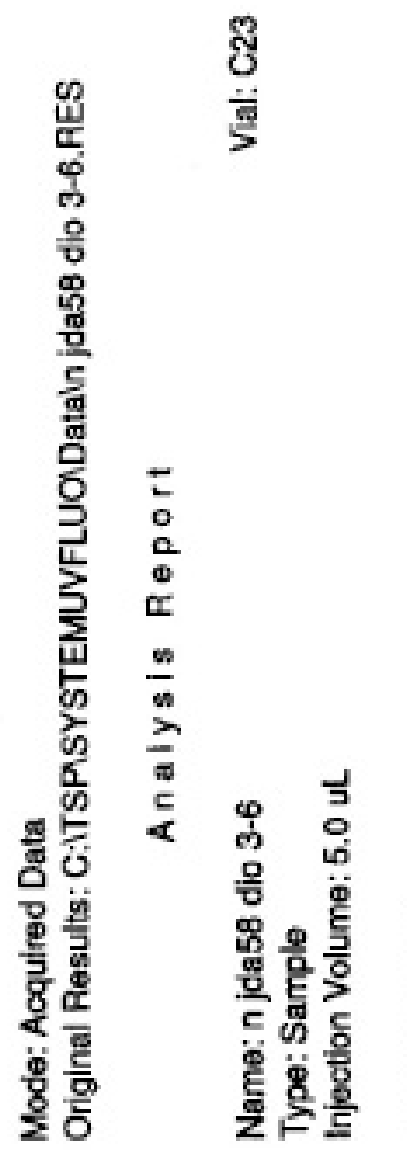

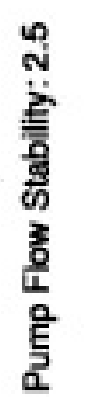
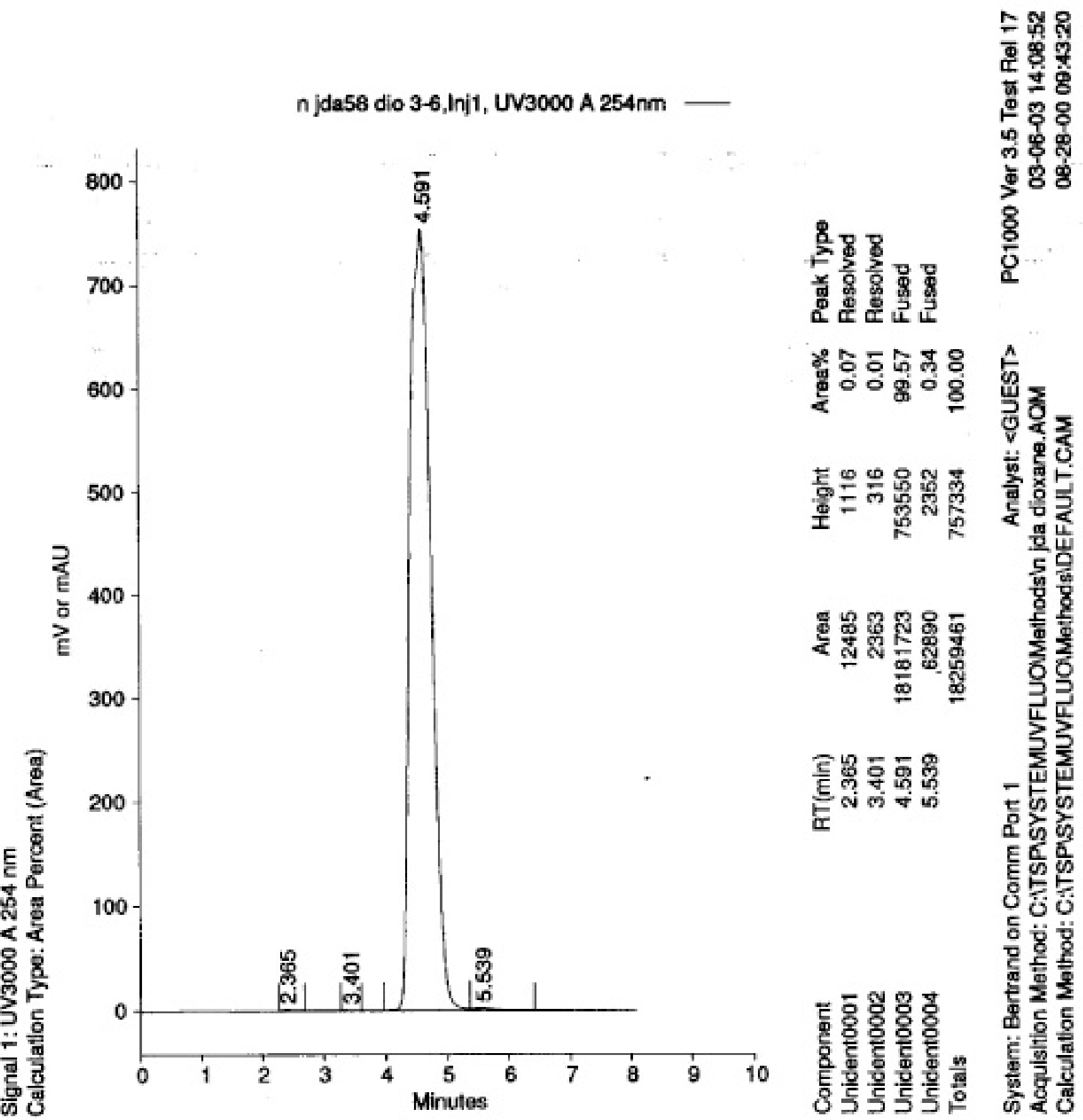
\#24 iprottidioxane 80:20

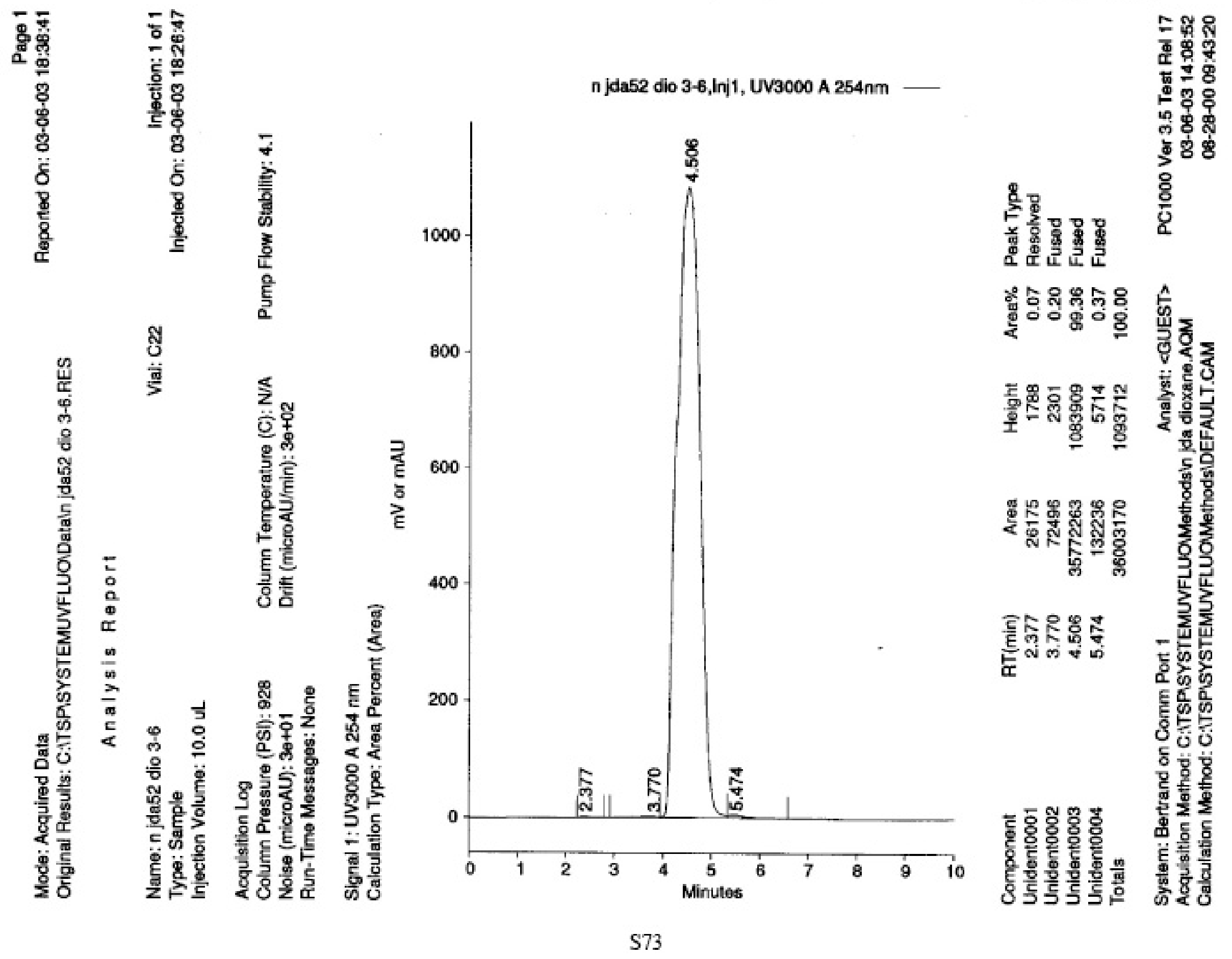

